\title{
Detection and Quantification of Neurotransmitters in Drosophila melanogaster by Capillary Electrophoresis- Fast Scan Cyclic Voltammetry
}

Madelaine Elise Denno Milford, New Hampshire Bachelor of Arts: Biochemistry, Mount Holyoke College, 2010

\section{$3 / 29 / 2016$}

A Dissertation presented to the Graduate Faculty of the University of Virginia in Candidacy for the Degree of Doctor of Philosophy

Chemistry Department

University of Virginia

May, 2016 
(C) Madelaine Elise Denno, 2016 


\section{Abstract:}

In this work, the development and use of capillary electrophoresis coupled to fastscan cyclic voltammetry (CE-FSCV) for the separation, detection, and quantification of neurotransmitters and their metabolites in Drosophila melanogaster tissues is discussed. In Chapter 1, the background material for this thesis: neurotransmitters, Drosophila as a model organism, separations of neurotransmitters, capillary electrophoresis, electrochemical detection, and past applications of CE-FSCV are explained. In Chapter 2, the application of CE-FSCV to the determination of tyramine, serotonin, octopamine, and dopamine in the central nervous system (CNS) of the larva, pupa, and adult Drosophila is discussed. Sex specific differences in neurotransmitter tissue content of the adult brain are also discussed. In Chapter 3, a new CE-FSCV method for the detection of histamine, the neurotransmitter required for vision in Drosophila, and its metabolite carcinine in a variety of Drosophila tissues is developed. Histamine and carcinine have not previously been analyzed by CE-FSCV, and this method allows direct detection of both of these analytes. Additionally, the detection and quantification of a metabolite of dopamine, $\mathrm{N}$ - $\beta$-alanyl dopamine, is possible using this method. Histamine, carcinine, dopamine, and $\mathrm{N}$ - $\beta$-alanyl dopamine content in a wild type strain along with two metabolic mutants is determined. This method provides the foundation for Chapter 4, where the tissue content of histamine and carcinine is determined in adult brains, larval central nervous systems, and adult eyes from visual mutants. Lastly, I have laid out future directions for the use of CE-FSCV and other methods to further understand the tissue content distribution of neurotransmission. 


\section{Dedication:}

To my family: thank you for your constant encouragement to pursue my interest in science from a very young age. I still haven't found any chicken fossils, but I'm also not an archeologist. Without the early support and help to build my interest, I have no idea what l'd be doing right now.

To my friends: thank you for being there to help and assist me with the difficult aspects of graduate school.

\section{Acknowledgements:}

For their assistance with Chapter 2, thank you to: Eve Privman

For their assistance with Chapter 3 thank you to: Eve Privman, Ryan Borman, and Danielle Wolin

For their assistance with Chapter 4 thank you to: Danielle Wolin, Eve Privman, and Ryan Borman

For assistance in the preparation of this work and all research contained herein, as well as mentoring, thank you to Dr. Jill Venton.

Additional thanks go to Dr. Jay Hirsh, whose lab provided some of the fly stocks used in this research. Dr. Hirsh also provided a number of helpful suggestions and ideas that were used in the preparation of this document. 


\section{Table of Contents}

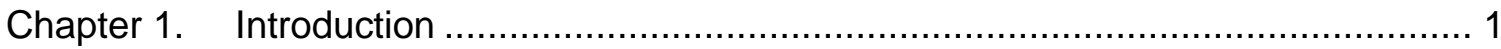

1.1 Neurotransmission and Neurotransmitters.............................................. 2

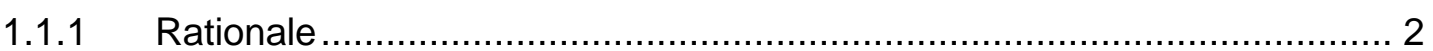

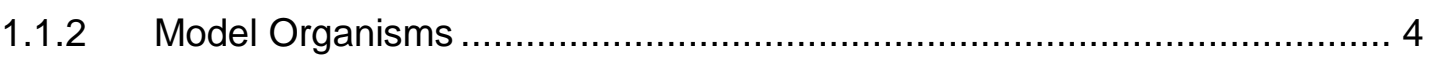

1.2 Separations for Neurotransmitters .................................................11

1.2.1 Gas Chromatography for Neurotransmitter Detection ............................12

1.2.2 High Pressure Liquid Chromatography for Neurotransmitter Detection.....12

1.2.3 Capillary Electrophoresis for Neurotransmitter Detection.........................14

1.2.4 Theoretical Considerations: HPLC and CE Separation Efficiency.............15

1.2.5 Detectors for Capillary Electrophoresis...........................................17

1.2.6 Electrochemical Detection in Capillary Electrophoresis ..........................21

1.2.7 Application of CE-FSCV to Separation and Detection of Neurotransmitters 25

1.3 Challenges for CE-FSCV Application to Drosophila..................................27

1.3.1 Drosophila as a Model Organism: Moving on from Larva, Tracking Development

1.3.2 Separations of Non-Catecholamine and Phenolamine Neurotransmitters and their Metabolites ......................................................................................

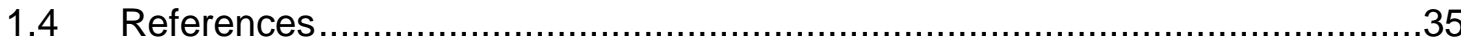

Chapter 2. Analysis of Neurotransmitter Tissue Content of Drosophila melanogaster in

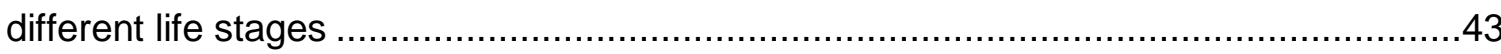

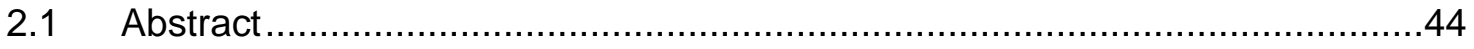

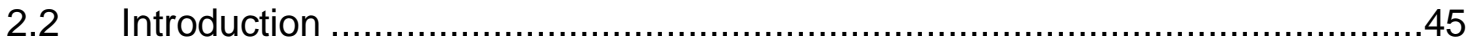

2.3 Results and Discussion ....................................................................... 46

2.3.1 Capillary electrophoresis and fast scan cyclic voltammetry ...................46

2.3.2 Separations of tyramine, serotonin, octopamine and dopamine................47

2.3.3 Separations of neurotransmitters in different life stages of Drosophila......48

2.3.4 Comparisons of neurotransmitters in different life stages of Drosophila....51

2.3.5 Comparisons of neurotransmitters in male and female adult Drosophila ..53

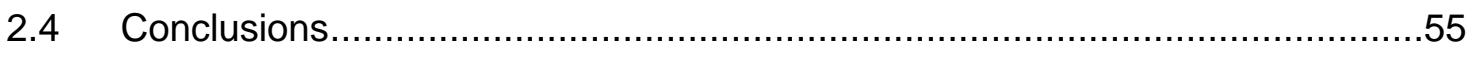

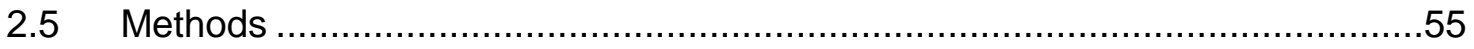




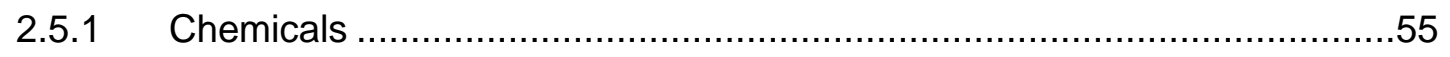

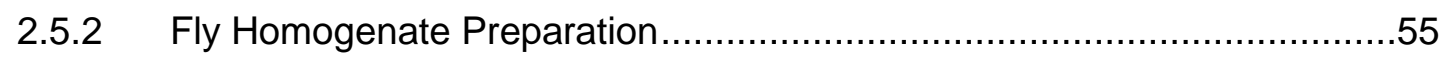

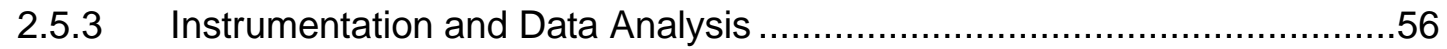

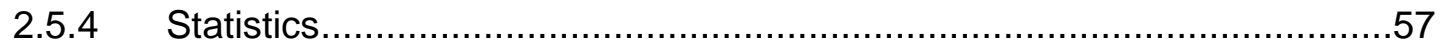

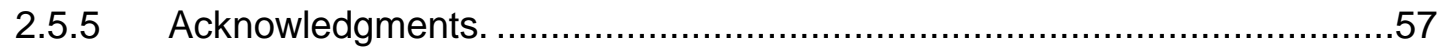

2.6 References .................................................. Error! Bookmark not defined.

Chapter 3. Quantification of histamine and carcinine in Drosophila melanogaster tissues 61

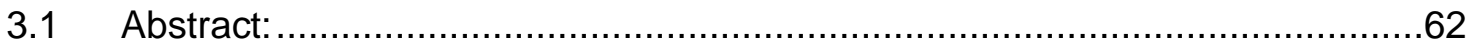

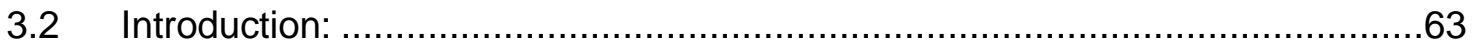

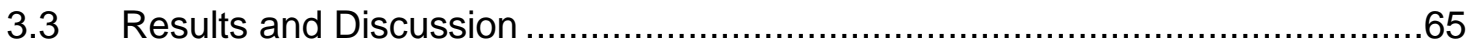

3.3.1 Capillary Electrophoresis with Fast Scan Cyclic Voltammetry for histamine

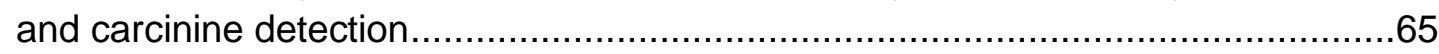

3.3.2 Quantification of Histamine, Carcinine, and Dopamine in the Brain, Eyes, and Cuticle of Drosophila ........................................................................68

3.3.3 Quantification of N- $\beta$-alanyl-dopamine in $\tan ^{3}$ flies ...............................75

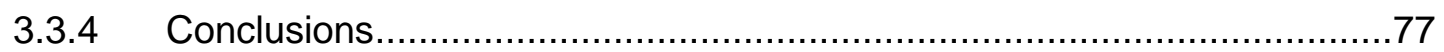

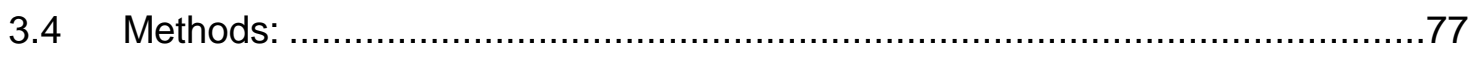

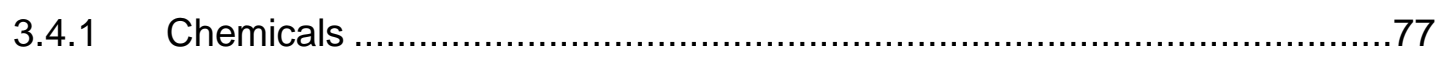

3.4.2 Capillary Electrophoresis ................................................................

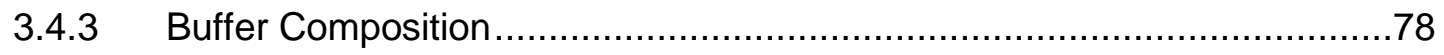

3.4.4 Fast Scan Cyclic Voltammetry and Data Collection ..................................79

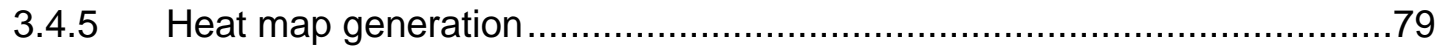

3.4.6 Drosophila melanogaster sample preparation …………………...........79

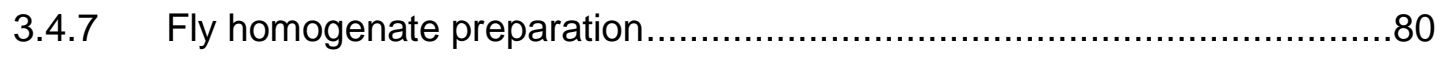

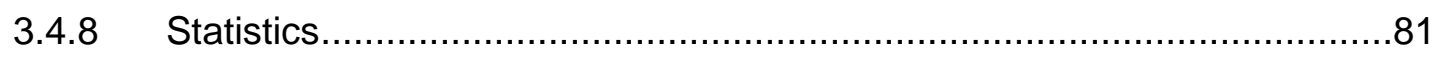

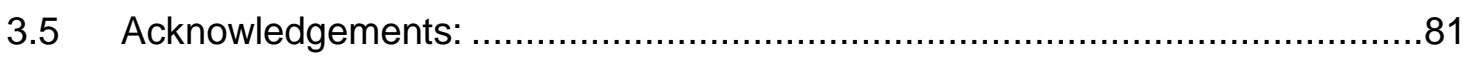

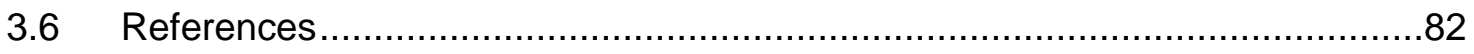

Chapter 4. Determination of Histamine and Carcinine Content in Drosophila

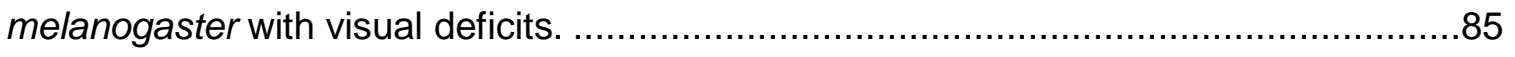

4.1 Abstract:

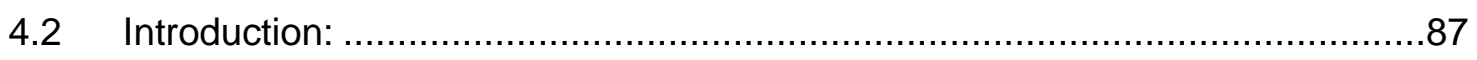

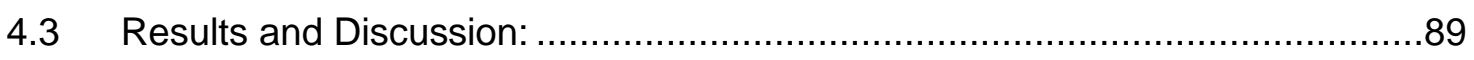

4.3.1 Drosophila melanogaster strains: Developmental Mutants and Eyesight..89 
4.3.2 Capillary electrophoresis for Histamine and Carcinine Detection. .90

4.3.3 Comparison of Tissue Content of Histamine and Carcinine in Different Tissues 92

4.3.4 Tissue Content of Histamine and Carcinine in Different Strains of Flies ...94

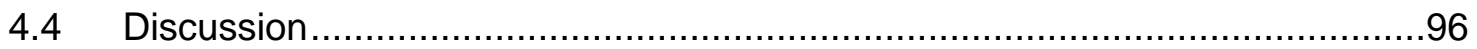

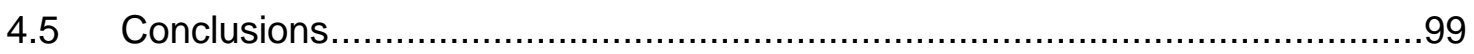

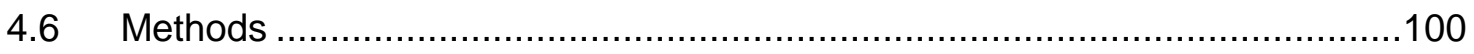

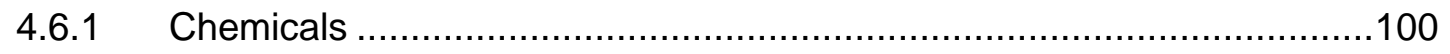

4.6.2 Capillary Electrophoresis ........................................................... 100

4.6.3 Buffer Composition for CE ......................................................... 101

4.6.4 Fast-Scan Cyclic Voltammetry and Data Collection.............................101

4.6.5 Drosophila melanogaster Tissue Sample Preparation ..........................102

4.6.6 Tissue Homogenate Preparation .....................................................102

4.6.7 Statistics............................................................................... 102

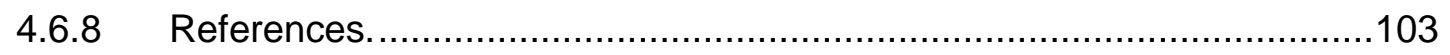

Chapter 5. Conclusions and Future Directions ..............................................106

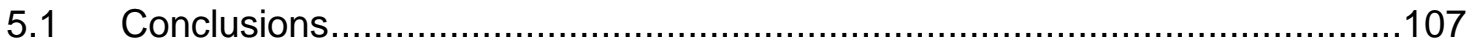

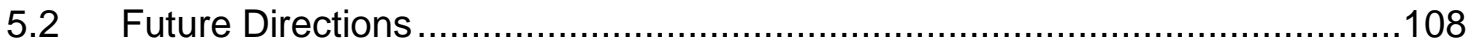

5.2.1 Tissue Content Monitoring in Aging Larvae and Adults .........................108

5.2.2 Analysis of $\beta$-alanine in Drosophila and Development of a CE-FSCV

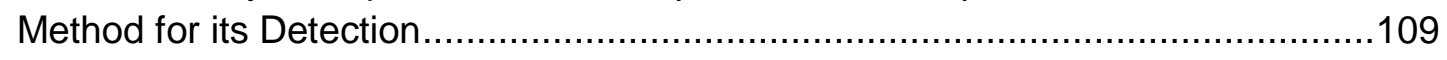

5.2.3 Histamine and Carcinine Content in Other Visual Mutants ....................110

5.2.4 Histamine and Carcinine Content in RNAi Suppressed Drosophila .........111

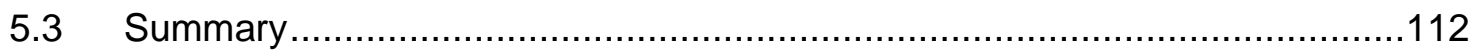

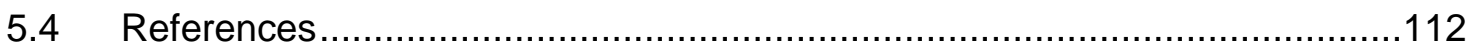




\section{Table of Figures}

Figure 1-1: Schematic of Neurotransmission ........................................................ 2

Figure 1-2: Schematic representation of synaptic and volume transmission involving dopamine in the central nervous system.............................................................. 3

Figure 1-4: The neuroanatomy of the adult fly brain.............................................. 5

Figure 1-3: Biogenic amines found in Drosophila melanogaster that have no known function in humans and are present at low concentrations........................................ 6

Figure 1-5: Optogenetic stimulation of Drosophila larval ventral nerve cord.................... 7

Figure 1-6: Characterization of dopamine signal evoked by ChR2 activation................. 9

Figure 1-7: Characterization of ATP evoked dopamine signal in P2X2 flies..................10

Figure 1-8: The principle of sample stacking in capillary electrophoresis.....................16

Figure 1-10: Decoupling methods for CE-EC:..........................................................19

Figure 1-11:Typical capillary-to-electrode alignments used for CE-EC without

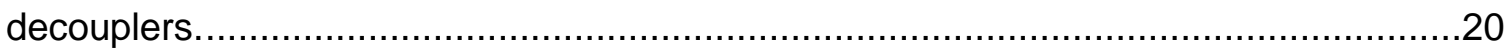

Figure 1-12: A triangular waveform as used in FSCV ...........................................23

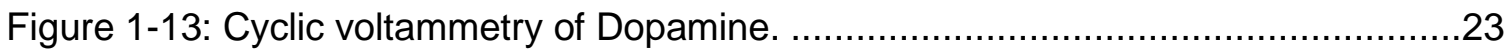

Figure 1-14: Capillary electrophoresis with Fast Scan Cyclic Voltammetry. ..................24

Figure 1-15: The larval Drosophila central nervous system. .......................................25

Figure 1-16: Separations of standards and fly tissue by CE-FSCV............................25

Figure 1-17:Separations of neurotransmitters found in rat brain tissue punches...........26

Figure 1-18: Life cycle of Drosophila melanogaster. ............................................27

Figure 1-19: The central nervous system of Drosophila melanogaster changes during

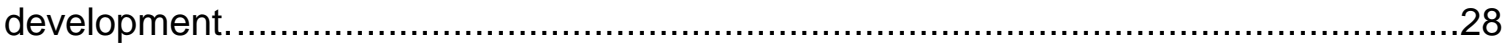


Figure 1-20: The role of Ebony and Tan in the metabolism of dopamine and histamine.

Figure 2-1: Cyclic voltammograms (CVs), electropherograms, and false color plots for separation of a $100 \mathrm{nM}$ standard mix.

Figure 2-2: Cyclic voltammograms (CVs), electropherograms, and false color plots for separations of various tissues.

Figure 2-3: Comparisons of average tissue content across the three tested life stages, larva $(n=8)$, pupa $(n=7-8)$, adult $(n=10)$. .52

Figure 2-4: Comparisons of tissue content in male $(n=5)$ and female $(n=5)$ adult Canton $S$ brains .54

Figure 3-1 Separations of a standard mixture of neurotransmitters: $100 \mathrm{nM}$ each of histamine (HA), carcinine (CA), dopamine (DA), and $\mathrm{N}$ - $\beta$-alanyl-dopamine (BADA) in 10 $\mu \mathrm{L}$ of $0.5 \mathrm{mM}$ perchloric acid. .66

Figure 3-2 Separations of histamine, carcinine, and dopamine from three tissue types. 67

Scheme 3-1 A simplified schematic of histamine metabolism. 69

Figure 3-3 Comparisons of tissue content in each tissue type for all strains. .71

Figure 3-4: Comparisons of ratios of histamine to carcinine ratios (HA:CA) for all three tissue types. 72

Figure 3-5: Separations of neurotransmitters in the eyes of a tan3 fly..... .75 Figure 3-6: Tissue content of $\mathrm{N}$ - $\beta$-alanyl-dopamine and ratios of dopamine to $\mathrm{N}$ - $\beta$-alanyldopamine in tan3. 76

Figure 4-1: Images of heads taken from adult A) Oregon R B) white ${ }^{1118}$ C) glass ${ }^{3}$ D) sine oculis $^{1} \mathrm{E}$ ) eyes absent ${ }^{2} \mathrm{~F}$ ) eyeless ${ }^{2}$ flies. .89 Scheme 4-1: A simplified schematic of the genetic regulatory network controlling eye formation in Drosophila. Genes highlighted in red represent the genes inactivated in mutants eye ${ }^{2}, \mathrm{so}^{1}$, and eya ${ }^{2}$ respectively. Double headed arrows indicate reciprocal 
regulation. Of these genes, toy, eye, eya, and dac alone can induce eye development. Based on a scheme presented by Wagner, $2007 .^{35}$ .90

Figure 4-2: Example separation of A) $100 \mathrm{nM}$ standards and B) $w^{1118}$ eye. In A) histamine (HA) can be seen on the color plot at $390 \mathrm{~s}$ and carcinine (CA) at $490 \mathrm{~s}$. In $\mathrm{B}$ ) histamine can be seen on the color plot at $390 \mathrm{~s}$ and carcinine can be seen at $490 \mathrm{~s}$. The cyclic voltammograms are shown on the right.

Figure 4-3: Comparison of tissue content of $A)$ histamine $(n=5-12)$ and B) carcinine $(n=$ 5-12) in tissues from all strains with eyes. .92

Figure 4-4: Comparison of tissue content of A) histamine $(n=11-12)$ and B) carcinine $(n=$ 11-12) in tissues from all strains without eyes. .93

Figure 4-5: Comparison of tissue content of histamine and carcinine in all of the different tissues tested. .94

\section{Table of Equations}

Equation 1: The Van Deemter equation.......................................................15

Equation 2: Variance and HETP in capillary electrophoresis. ................................15

\section{Table of Tables}

Table 2-1: Average tissue content values in larva, pupa and adult. .50 Table 3-1: Tissue content per milligram of tissue of histamine, carcinine, and dopamine in brain, eyes, and cuticle. .70

Table 4-1: Tissue content for all tissues tested in all strains of flies. .96 
Chapter 1. Introduction 


\subsection{Neurotransmission and Neurotransmitters}

\subsubsection{Rationale}

Understanding the functions of neurotransmitters and neurotransmission is a difficult, but important task due to the wide variety of neurological diseases and disorders. These can range from depression to Alzheimer's disease, and may be either mild or debilitating to the individual. These diseases generally take a large toll not only on the person diagnosed with them, but on their caregivers, family, and the economy at $\operatorname{large}^{1}$. While these diseases and disorders are increasingly well understood, there are still many questions about the fundamental functions of the brain and the ways that these diseases develop. This knowledge gap can limit the development of treatments. As such, further development of methods for understanding neurotransmission is required. These methods are generally tested first in animal models to determine their utility and suitability. The mechanisms of neurotransmission in these model organisms can be very similar to humans, and there may be homologous genes known to be involved in human diseases, even in very simple model organisms, such as D.melanogaster. Even though these model organisms may appear to be very different, the fundamentals of Figure 1-1: Schematic of Neurotransmission

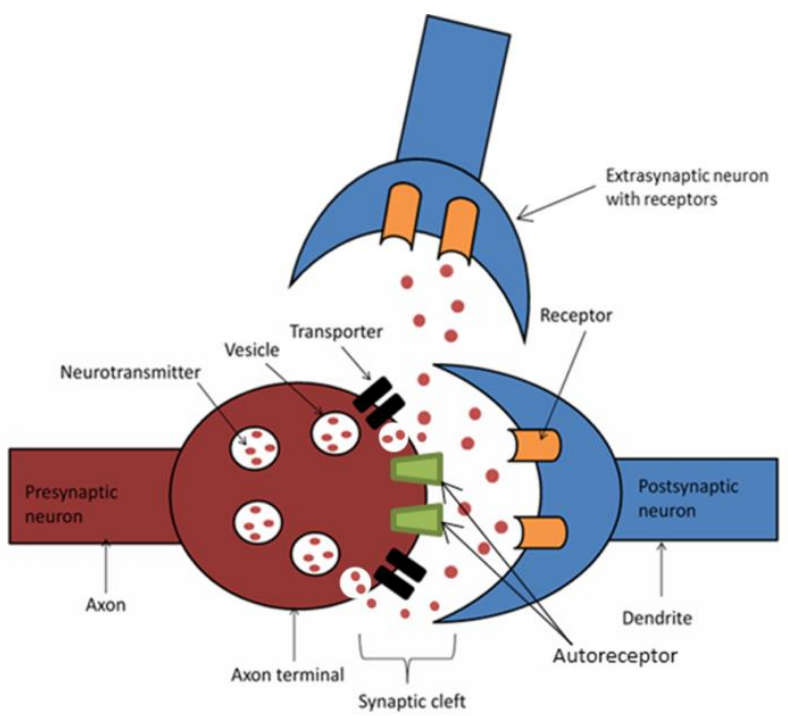

A simplified schematic depicting neurotransmission. On the left, the presynaptic neuron, releases neurotransmitters that have been packaged in vesicles into the synaptic cleft. These neurotransmitters then bind to the receptors on the postsynaptic neuron, activating it. Above, neurotransmitter diffuses away from the synaptic cleft, where it arrives at an extrasynaptic neuron, where it may bind to a receptor. 
neurotransmission remain the same.

During neurotransmission, a presynpatic neuron releases signaling molecules (neurotransmitters) into the synaptic cleft where they then can bind to receptors on the postsynaptic neuron, diffuse away, bind to auto-receptors on the pre-synaptic neuron, undergo enzymatic degradation, or undergo reuptake. If the released neurotransmitter

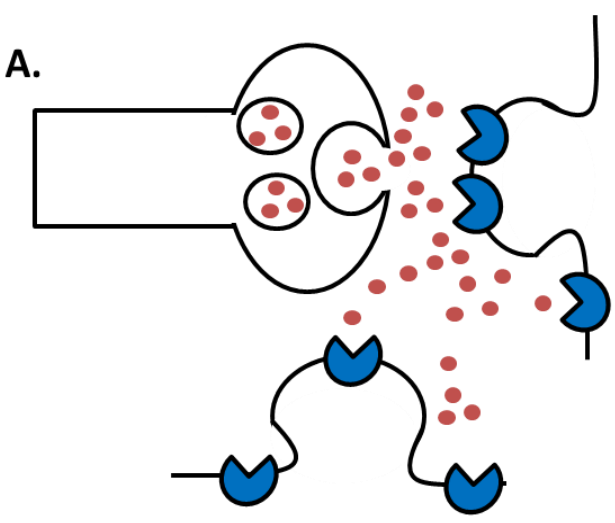

Intrasynaptic vesicular release (spillover from synapse)

B.

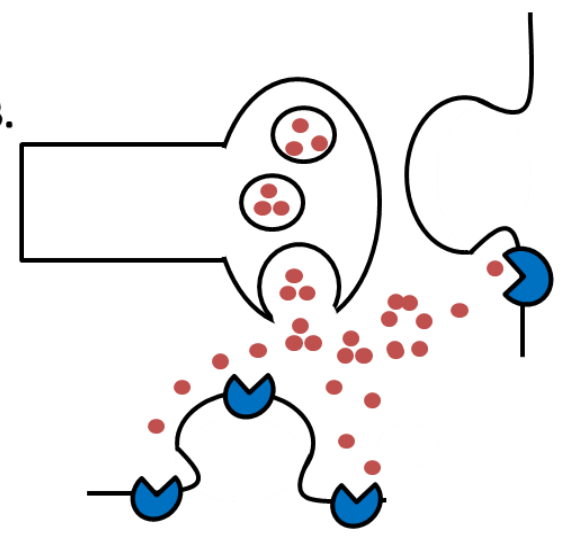

Extrasynaptic vesicular release

Figure 1-2: Schematic representation of synaptic and volume transmission involving dopamine in the central nervous system.

a) Intrasynaptic vesicular release of dopamine into the synaptic cleft; spillover of the released dopamine will allow dopamine to diffuse outside of the synaptic cleft. If the concentration is high enough, extrasynaptic receptors or receptors in neighboring synapses can be activated. b)Extrasynaptic vesicular release: both the release site and the receptor are outside of the synapse. binds to a receptor on the postsynaptic neuron, the postsynaptic neuron may either be influenced in an inhibitory or excitatory way, and may activate downstream signaling pathways.(Figure 1). If the neurotransmitter diffuses away, or if a large concentration of neurotransmitter is released and spills overfrom the synaptic cleft, it can then go on to activate extrasynaptic receptors. This is volume transmission, which functions similarly in that it activates target cells via extrasynaptic receptors (Figure $2 \mathrm{~A})^{2-4}$. Other forms of volume transmission exist, such as extrasynaptic vesicular release (Figure 2B) where the release of neurotransmitter and the target receptor are both extrasynaptic. 
Measurement of synaptic neurotransmission is extremely difficult due to the small size of the synapse and but some information can be gained by patch-clamp recordings of neurons done in cell cultures or more rarely, in vivo ${ }^{5}$. Patch clamp is generally performed on large neurons, and therefore can be limited in the types of neurons it can record from. However, patch clamp gives the firing rate of the patched neuron, and does so with high time resolution. ${ }^{6}$ In some cases, direct measurements of vesicular release from cultured or isolated cells are possible, but these measurements are limited to a small number of cell types, again primarily from larger neurons or clusters of neurons. ${ }^{7,8}$ In vivo studies of neurotransmission, such as those using microdialysis, detect and are able to monitor volume transmission. ${ }^{9}$ While measuring synaptic transmission is important, volume transmission is a major mode of action for neurotransmitters such as dopamine and serotonin.

In addition to information on the dynamics of neurotransmitter release, the neurotransmitter content of a tissue can be important. Neurotransmitter tissue content is dependent on releasable pools of neurotransmitter, tissue dependent expression of genes controlling neurotransmitter synthesis and metabolism, and the age or developmental status of the tissue. ${ }^{10,11}$ Tissue content on its own gives an incomplete picture of neurotransmission. For a complete picture of neurotransmission and brain function, tissue content must be understood alongside neurotransmitter kinetics, metabolism, and release. Basic studies on tissue content and neurotransmission have been performed in a wide variety of model organisms.

\subsubsection{Model Organisms}

Two of the most widely used model organisms are mice and rats. Both are fairly prolific reproducers with short generation time, can be kept in colonies, and have been bred to produce a high level of genetic homogeneity ${ }^{12}$. Mice are popular due to the wide 
availability of knock-out or

knock-in mice, transgenic

mice, and other genetic

modifications that are more

difficult to do in rats. However, despite their easy genetics, mice are less physiologically similar to humans than rats and have great difficulty learning to perform tasks. ${ }^{13}$

Furthermore, their smaller
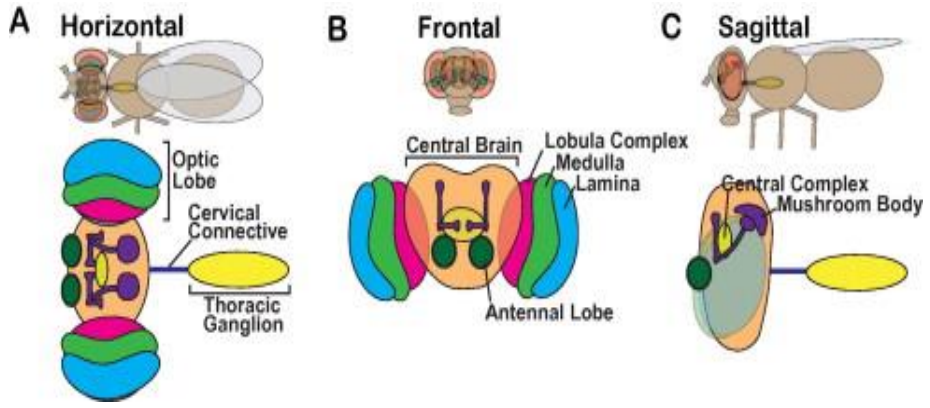

Figure 1-3: The neuroanatomy of the adult fly brain.

Cartoons depict A) Horizontal B) frontal and C) sagittal views of the fly and the central nervous system. Structures are labeled and color coded. Labeled structures are the central brain (orange), optic lobes, the thoracic ganglion (yellow), and the cervical connective (blue). The optic lobes are further broken down into lamina (aqua), medulla (green), and lobula complex (magenta). Major brain structures within the central brain are the mushroom bodies (purple) and the central complex (yellow circle). Reprinted (adapted) with permission, from Krantz et al. ${ }^{19}$

size makes it more difficult to determine how the brain is altered in response to stress and other influences. Due to these factors, rats are more popular for studying the brain and neurotransmission. ${ }^{12}$ Despite the advantages of using rodent models, maintaining breeding populations can be costly and time consuming. Additionally, there are complex regulations for research involving mice and rats.

Increasingly, Drosophila melanogaster has become popular as a model organism because it avoids some of these limitations. ${ }^{14,15}$ Flies have a rapid reproductive cycle, short generation time ( 2 weeks), easily manipulated genetics, fully sequenced genome ${ }^{16}$ and wide variety of available mutants. Some mutants are available that mimic human diseases. For example, Drosophila models have been made for Parkinson's disease, Alzheimer's, circadian rhythm disorders, and restless legs syndrome. ${ }^{17}$ It is estimated that approximately $60 \%$ of human disease genes have an ortholog in Drosophila. 
Despite its small size, the central nervous system of Drosophila is well characterized and mapped (Figure 3). ${ }^{18}$ Even though there are a number of apparent differences between Drosophila and mammals, Drosophila have dopaminergic and serotonergic signaling, and these systems operate similarly to those found in humans. ${ }^{19}$ Histamine is also found in Drosophila and is responsible for circadian rhythm, sleep, wakefulness and clock keeping, similar to humans. ${ }^{20,21}$ In addition to these shared neurotransmitters, Drosophila also has several other neurotransmitters and biogenic amines that are found only in trace amounts in humans, such as octopamine, tyramine, and carcinine (Figure 4). Despite the differences in structure of the neurotransmitters octopamine and tyramine, they are reported to have homologous functions to the human neurotransmitters norepinephrine and epinephrine. ${ }^{22}$<smiles>NCCc1cccc(O)c1</smiles>

Tyramine<smiles>NCC(O)c1ccc(O)cc1</smiles>

Octopamine<smiles>NCCC(=O)NCCc1cnc[nH]1</smiles>

Carcinine

Figure 1-4: Biogenic amines found in Drosophila melanogaster that have no known function in humans and are present at low concentrations.

Tyramine is a precursor for octopamine synthesis in the fly. Carcinine is a metabolite of histamine in the fly.

Some of these shared neurotransmitter systems may also perform other important roles in Drosophila. Histamine, for example, is also important for visual processes in Drosophila, but does not participate in the visual processes of humans. As such, careful study and monitoring of the neurotransmission and neurotransmitters in Drosophila are important to understand not only the ways in which Drosophila is an adequate model organism, but also to understand the ways in which it differs from humans. 


\subsubsection{In vivo monitoring of neurotransmission in Drosophila melanogaster}

Similar to stimulated release work done in rats, it is also possible to stimulate the release of a variety of different neurotransmitters in the brain and central nervous system (CNS) of Drosophila. These

may be obtained from larva

or from adult Drosophila.

There are structural

differences between the adult

and larval CNS due to the

metamorphosis that

Drosophila undergoes.

A.

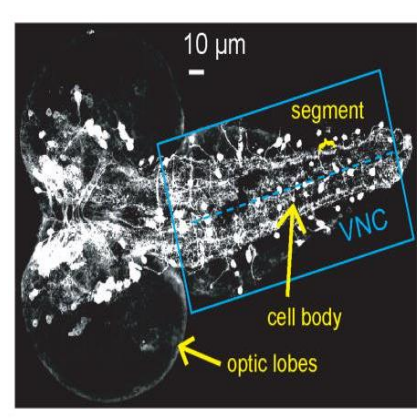

B.

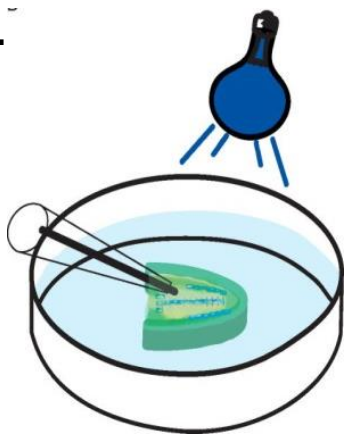

Figure 1-3: Optogenetic stimulation of Drosophila larval ventral nerve cord

A) Fluorescence microscopy image of GFP-labeled dopaminergic neurons in a 5-day-old, 3rd instar larva CNS. The blue box indicates the ventral nerve cord, and the dashed line marks the midline, where many cell bodies (white circles) are located. On either side of the midline is the neuropil region, which is rich in dopamine terminals. B) Schematic of microelecrode placement into the neuropil region of the ventral nerve cord with blue-light stimulation. The optic lobes have been removed from the CNS. Reprinted (adapted) with permission from Vickrey et al. 2009. ${ }^{25}$

addition to CNS preparations

of Drosophila, it is possible to obtain stimulated release of neurotransmitters in

preparations where the neuromuscular junction is exposed (larval fillet preparation), or in other tissues where neurotransmitters may be present (such as the gut). ${ }^{23}$ Stimulated release in Drosophila is not done by electrical stimulation, as it is often done rats and mice, but can be accomplished through a variety of different methods.

\subsection{Optogenetic stimulated release}

One of the most popular and versatile methods for stimulated release is optogenetics, which uses light sensitive channels (typically channelrhodopsin or a related protein) expressed in cells of interest. ${ }^{23,24}$ This expression is most commonly controlled by the UAS-Gal4 system, which allows expression of Channelrhodopsin in neurons that express certain genes which are related to the synthesis of the target 
neurotransmitter. ${ }^{19}$ For example, to target channelrhodopsin 2 (ChR2) expression to dopaminergic cells, a UAS-Gal4 TDC driver may be used. When these targeted cells are illuminated with a specific wavelength of light, the channels are activated and they open and allow cations into the cell. In neurons, this leads to depolarization and release of the targeted neurotransmitter.

Early work in our lab focused on the use of blue light stimulated channelrhodopsins for release of neurotransmitters in the larval ventral nerve cord (VNC). Channelrhodopsin 2 was expressed in dopaminergic and serotinergic neurons and the VNC was stimulated using blue light (Figure 5). Release of the targeted neurotransmitter was detected using fast scan cyclic voltammetry (FSCV). ${ }^{25-28}$ Detection of stimulated release by FSCV also allowed for determination of kinetics for uptake and clearance, determination of the releasable pool of a neurotransmitter, and the effects of drugs on these parameters. In comparison to "puff-on" work, where the clearance and uptake of exogenously applied neurotransmitter at high or higher than physiological concentration is determined, this allowed for more physiologically relevant determinations of receptor kinetics and the impact of drugs on these kinetic parameters. 

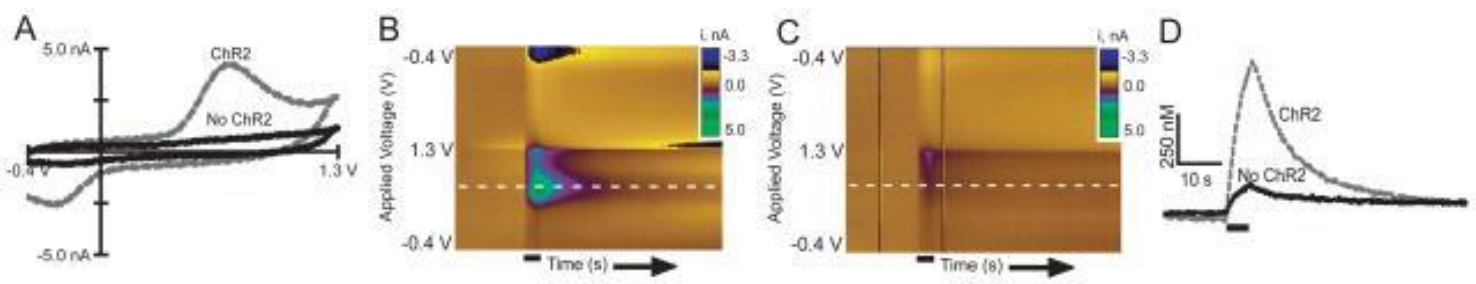

Figure 1-4: Characterization of dopamine signal evoked by ChR2 activation.

A) Background subtracted cyclic voltammograms from a ChR2 expressing larva (grey) and a control larva (black). B) Color plot of blue light stimulated dopamine release in ChR2 expressing larva. C) Color plot of blue light stimulated control larva- while there is no dopamine signal visible, there is clearly a change in current at the switching potential caused by blue light application. D) Current vs. time plot showing that the dopamine signal in ChR2 larva increases with blue light application and then decays after the light is turned off. The control larva shows and increase as well, due to ionic changes. Reprinted (adapted) with permission from from Vickrey et al. 2009. ${ }^{25}$

While these stimulations were a huge improvement over puff-on methods, blue light stimulation proved challenging when used in conjunction with FSCV: the high energy and high intensity blue light stimulation caused changes in the surface of the electrode, which made detection of other neurotransmitters difficult (Figure 6). With the emergence of newer channelrhodopsins that are activated by higher wavelength light (red and orange stimulated CsChrimson), further characterization of dopamine has been possible..$^{29,30}$ In addition, the reduced photoelectric effect from red light stimulations has made it possible to monitor the release and kinetics of compounds that oxidize at higher potentials. For example, octopamine's primary oxidation peak ( $1.3 \mathrm{~V}$ vs $\mathrm{Ag} / \mathrm{AgCl})$ occurrs close to the signal caused by blue light stimulation, which would make accurate identification and quantification difficult.

\subsection{Other methods of stimulated release}

Other methods of stimulated release in Drosophila have also become available recently. $P 2 X_{2}$ is a ligand-gated ion channel that opens in response to ATP, and strains of flies expressing $\mathrm{P}_{2} \mathrm{X}_{2}$ in a variety of neurons are available. These channels are not natively expressed or present in Drosophila, and as such, there is no concern of activation of non-selectively expressed $\mathrm{P} 2 \mathrm{X}_{2}$ These channels require the application of 
exogenous ATP to stimulate release of neurotransmitters (Figure 7A). ${ }^{31}$ While ATP is electroactive, the higher potentials needed to detect it by FSCV (1.45 V vs. Ag/AgCl) prevent interference by ATP during detection of neurotransmitters (Figure 7B). Dopamine release and the releasable pool of dopamine in larval VNCs were determined using this method, and this method was determined to be comparable to optogenetic methods for stimulated release. Other novel applications of this mode of stimulated release combines optically caged ATP with expression of $\mathrm{P}_{2} \mathrm{X}_{2}$, allowing for extremely short duration and specific stimulation of the tissue, and allowing for stimulation of behaving flies. ${ }^{24}$

(a) Larval VNC expressing $\mathrm{P}_{2} \mathrm{X}_{2}$

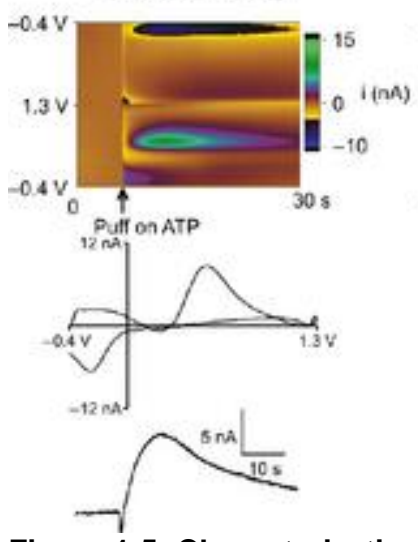

Figure 1-5: Characterization of ATP evoked dopamine signal in P2X2 flies.

Top row: false color plots with time on the x-axis, applied voltage on the $y$-axis, and Faradaic current in pseudocolor. Injection of ATP is denoted by the arrow under the figure. Middle row: background-subtracted cyclic voltammograms. Bottom row: signal traces show dopamine current changes over time. (a) 2 pmol ATP was injected into a larval ventral nerve cord (VNC) expressing P2X2 and the color plot and CV show that dopamine is released upon ATP stimulation. (b) 2 pmol ATP was injected into a control larval VNC without P2X2 expression and the color plot shows minor fluctuations upon ATP injection corresponding to pressure changes, but the CV does not show any characteristic dopamine peaks. Reprinted (adapted) with permission from Xiao et al $2015 .{ }^{31}$
These in situ and in vivo

methods are important for

understanding release and clearance of neurotransmitters in

Drosophila, as well as

understanding the impact of drugs

on these parameters. It also

provides information on local

receptor density and density of

release sites in the area where

measurements are being taken.

Furthermore, in situ

measurements of

neurotransmitters provide

information on releasable pools

and replenishment of these pools. 
However, they cannot account for the total amount of neurotransmitter present in the tissue, and due to their high spatial resolution, only give local information on releasable pools. In order to understand the total tissue content of neurotransmitters, differences in neurotransmitter storage in mutants, or differences in total tissue content between life stages, tissue content analysis must be performed. This is generally achieved through the use of separations, which are necessary due to the wide variety of compounds present in tissues.

\subsection{Separations for Neurotransmitters}

In all of the discussed model organisms, samples can be obtained from blood (or hemolymph in flies), urine, or brain tissue and are generally fairly complex, containing a variety of neurotransmitters or other compounds. Samples are often prepared for analysis through separatory methods such as gas chromatography (GC), high pressure liquid chromatography (HPLC), and capillary electrophoresis (CE).

Separations of complex mixtures exploit a differential displacement, which in order to separate components, must have selective movement of one component with respect to another component. Broadly, these displacements can be classified as bulk flow displacements and relative displacements. Flow displacement can arise by the movement of medium containing components of interest through or across a container or tubing, and can include flow over packed beds, such as a packed column used in liquid chromatography. Relative displacement is caused by the motion of a component through the medium. This movement can be caused by the application of a force or a gradient; an applied electrical field can drive charged species (as in capillary electrophoresis) or buffer gradients (in high pressure liquid chromatography). Both types 
of displacement may be combined to achieve separation. In both cases, equilibria drive separative processes, regardless if they are chromatographic or electrophoretic.

\subsubsection{Gas Chromatography for Neurotransmitter Detection}

Early on, gas chromatography (particularly with mass spectrometry detection (MS)) was used for detection and quantification of neurotransmitters in tissue, however analysis times were long for multiple samples and samples required derivatization to be made volatile, both for GC as well as MS detection ${ }^{32}$. Volatile samples are required for gas chromatography, as separation in GC relies on the interaction of analytes in the gas phase interacting with the stationary phase coating the inside of the capillary.

Temperature ramps and gradients are used to increase separation and improve peak resolution, and occasionally, carrier gases may be altered to increase sensitivity and column efficiency. In these early experiments, the expense of GC or GLC-MS made it difficult to use GC-MS for routine tissue content determination and other GC methods suffered from poor sensitivity ${ }^{33}$. Early GC-MS also frequently required the use of selected ion monitoring, which reduced the scope of experiments from detecting and quantifying what was present in the sample to what was expected in the sample ${ }^{34}$. GC methods are still in use today, but have been largely replaced by HPLC methods.

\subsubsection{High Pressure Liquid Chromatography for Neurotransmitter Detection}

High pressure liquid chromatography (HPLC) is popular due to its high resolving power, the wide variety of columns available, and the detectors available. HPLC uses packed columns, which contain a stationary phase which interacts with analytes. Separation is based on the difference in analyte retention time in the stationary phase. Stationary phases can be non-polar (reverse phase), polar (normal phase), or chiral, with the column packing selected to match the polarity of the analytes of interest. In addition to the stationary phase, the mobile phase is also tailored to the separation: in normal phase they are non-polar and in reverse phase they are polar. Mobile phases 
may also be altered by the use of gradients. Reverse phase HPLC is the most commonly employed mode of HPLC and is frequently used to determine neurotransmitter content in samples. ${ }^{35}$

HPLC systems for neurotransmitter detection commonly employ fluorescence and UV-Vis detection, which could require the derivatization of analytes, or electrochemical detectors, which do not. HPLC systems can also be coupled to mass spectrometry or MS-MS, typically with electrospray ionization ${ }^{36}$ or atmospheric pressure chemical ionization $^{37}$. Derivatization of analytes for LC-MS may improve sensitivity, and is therefore widely used. ${ }^{36,38}$ Electrochemistry is one of the most widely used methods of detection for HPLC separations involving neurotransmitters. Electrochemical detection is typically coulmetric ${ }^{39}$ or amperometic ${ }^{40,41}$ and rarely voltammetric ${ }^{42}$. Amperometric detectors for HPLC apply a constant potential sufficient to oxidize analytes of interest. The typical operating range for these detectors is $0.65-0.8 \mathrm{~V}$, and dual electrode systems may be used ${ }^{43,44}$ for better separation of peaks or increased sensitivity. Initially, HPLC-EC was applied to the analysis of neurotransmitters obtained from tissue samples $^{40,45}$ (such as mouse or rat brain homogenates or spinal cords). Total tissue content of neurotransmitters was analyzed, and when tissue samples were taken from discreet brain regions, regional variations and tissue content could be determined ${ }^{46}$. Urine $^{47}$, serum and cerebral spinal fluid ${ }^{48,49}$ collected from animals or humans were also tested for neurotransmitter content.

Sample collection was initially offline and analysis times (around 10 minutes at minimum) long enough that real time tracking of serum or CSF neurotransmitter concentration is extremely difficult. The use and development of microdialysis (a form of push pull perfusion) allowed for on-line sampling ${ }^{50-52}$ of CSF concentrations of neurotransmitters, but separation times for HPLC remained long compared to the 
timescale on which changes in neurotransmission occur. Shorter separations, on the 1-2 minute time scale are possible with recent advancements in ultra-high pressure liquid chromatography, but are not routinely performed. ${ }^{53,54}$ Furthermore, relatively large samples had to be collected for HPLC analysis, as sample volumes typically are in the 10-25 $\mu \mathrm{L}$ range. Despite these challenges, on-line microdialysis coupled to HPLC has become popular and widely used for analysis of neurotransmitters from CSF.

\subsubsection{Capillary Electrophoresis for Neurotransmitter Detection}

The development of capillary electrophoresis for separations in the 1980s introduced the possibility of faster separations. Capillary electrophoresis was first reported in 1959 by Hjerten ${ }^{55}$. Smaller capillary sizes ${ }^{56,57}$ and the wider availability of fused silica capillaries made capillary electrophoresis more attractive for wider use than earlier iterations. Early separations using capillary electrophoresis were comparable in length to shorter HPLC methods (with separations being completed in around 10 minutes), but CE was easily adapted to homebuilt systems, separations could be tailored by changing buffering systems rather than both column and buffer, and small sample volumes were easily analyzed. Several modes of capillary electrophoresis exist, such as capillary zone electrophoresis (CZE), micellar electrokinetic chromatography (MEKC), capillary isoelectric focusing (CIEF), and capillary gel electrophoresis (CGE) ${ }^{58,59}$. Of these methods, CZE is best suited to small molecule separations. In CZE, speed of separation is modified by changing the applied separation voltage and the length of the capillary. Shorter capillaries and higher applied separation voltages lead to shorter separation times. Separations on the microsecond time scale have been reported, but are generally not used for analysis of neurotransmitters. ${ }^{60}$ For neurotransmitters, separations as short as 12 seconds have been reported, with a field strength of 1.75 $\mathrm{kV} / \mathrm{cm}$ over a $4 \mathrm{~cm}$ separation capillary; however these parameters are not generally suitable due to Joule heating and other related phenomena. ${ }^{61}$ Selectivity and retention 
times can easily be modified through buffer selection ${ }^{57}$ and the addition of modifiers, such as organic solvents, complexants ${ }^{62}$, and surfactants ${ }^{63}$.

\subsubsection{Theoretical Considerations: HPLC and CE Separation Efficiency}

In HPLC, band broadening and separation efficiency are influenced by eddy

diffusion (A-term), longitudinal diffusion (B-term), and resistance to mass transfer (Cterm) (Equation 1). In CE, HETP is defined by different considerations. The total variance $\left(\sigma^{2}{ }_{\text {tot }}\right)$ is caused by Joule heating $\left(\sigma_{T}^{2}\right)$, diffusion $\left(\sigma_{\text {diff }}^{2}\right)$, wall interactions $\left(\sigma_{\text {wall }}^{2}\right)$, and sample introduction $\left(\sigma^{2}\right.$ int $)$ (Equation 2). In many cases, these factors can be easily minimized or eliminated altogether by controlling capillary temperature, careful loading of the capillary to avoid over- or underfilling, and pretreatment of the capillary wall or the use of certain buffers to avoid interactions between analytes and the side walls. These properties and developments made capillary electrophoresis an attractive method for separations of biological samples.

$$
\operatorname{HETP}=A+\frac{B}{u}+\left(C_{s}+C_{m}\right) * u
$$

\section{Equation 1: The Van Deemter equation.}

The height equivalent to a theoretical plate (HETP), is defined by several terms: $A$, the Eddy-diffusion parameter; B, the diffusion co-efficient of eluting species in the longitudinal direction, which causes dispersion; $C$, resistance to mass transfer of the analytes between the mobile $(\mathrm{m})$ and stationary $(\mathrm{s})$ phase; $\mathrm{u}$, the

$$
\begin{gathered}
\text { a) } \sigma^{2}=\sigma_{\text {diff }}^{2}+\sigma^{2}{ }_{T}+\sigma^{2}{ }_{\text {int }}+\sigma^{2}{ }_{\text {wall }} \\
\text { b) } \mathrm{HETP}=\frac{L}{N}=\sigma^{2}{ }_{\text {tot }} / L
\end{gathered}
$$

\section{Equation 2: Variance and HETP in capillary electrophoresis.}

a) Total variance in capillary electrophoresis separations $\left(\sigma_{\text {tot }}^{2}\right)$ arises from Joule heating $\left(\sigma^{2}\right)$, diffusion $\left(\sigma_{\text {diff }}^{2}\right)$, wall interactions $\left(\sigma^{2}\right.$ wall $)$, and sample introduction $\left(\sigma^{2}\right.$ int). This is used to determine HETP $(b)$, which is also dependent on the length of the capillary $(L)$. 
Even early on, CE found applications in the analysis of neurotransmitters ${ }^{63-65}$, especially from small, low concentration samples of neurotransmitters. For example, single cells were analyzed using capillary zone electrophoresis $(\mathrm{CZE})^{64}$, microdialysis samples obtained from animals were analyzed ${ }^{66,67}$, and tissue samples studied ${ }^{68,69}$. CE worked well for these applications as it can handle small sample volumes (less than 10 $\mu \mathrm{L})$.

\subsubsection{Other Factors Impacting Separation Efficiency: Capillary Electrophoresis with Sample Stacking}

Capillary electrophoresis can easily be coupled to sample enrichment techniques such as sample stacking $^{70}$, isotachophoresis ${ }^{62,71}$, or isoelectric focusing ${ }^{72}$. A combination of

A

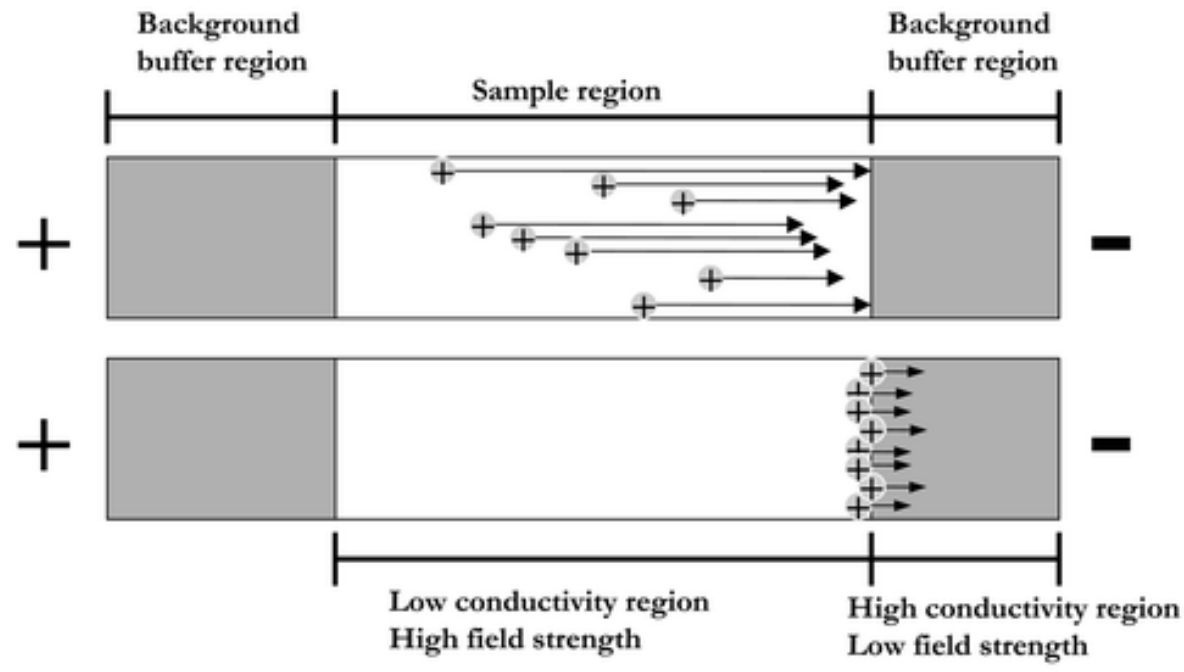

Figure 1-6: The principle of sample stacking in capillary electrophoresis.

A) A sample plug of cations is injected in a low conductivity buffer (such as de-ionised water). When voltage is applied, the electric field in the sample solution is higher than the rest of the capillary, cations migrate rapidly through the sample zone until they reach the low electric field in the separation buffer. B) The cations then slow down and stack at the boundary between the sample region and the buffer region. Reprinted (adapted) with permission from de Mello et al. ${ }^{73}$

sample stacking modes is also possible, with careful buffer design and selection.

Sample stacking arises from high electrophoretic mobility of analytes in low ionic strength backgrounds. ${ }^{73}$ After the application of voltage to the capillary, analytes in a low conductivity environment will accelerate to a boundary between the low ionic strength 
environment and a higher ionic strength environment (such as the separation buffer) (Figure 8A). After crossing the boundary, the mobility of analytes will be reduced in the higher ionic strength background; this causes "stacking" of analytes in the sample into a smaller zone than they were in when injected (Figure 8B). The ionic strength gradient dissipates shortly thereafter, and the charged analytes in the "stacked" zone migrate toward the cathode. This form of stacking can be incorporated easily and yield a 10-fold enhancement in sample concentration.

Isotachophoresis functions in a similar manner, exploiting differences in ionic mobility. Generally, higher ionic mobility and lower ionic mobility electrolytes are used to sandwich analytes of intermediate mobility between them after the application of an electrical field. This sandwiching causes concentration and focusing of analytes at the leading and terminating electrolyte interface. Isoelectric focusing is more popular for peptides and proteins, where distinct pls (isoelectric points) of the macromolecule allow them to be separated by $\mathrm{pH}$ gradients. The analytes will migrate rapidly through the capillary as voltage is applied through $\mathrm{pH}$ gradients until they reach a $\mathrm{pH}$ area that matches their pl, where they then migrate with that background electrolyte. Other varieties of preconcentration, such as filtration, electrocapture, or chromatographic preconcentration may also be used, but can greatly increase the complexity of the separation and sample handling.

\subsubsection{Detectors for Capillary Electrophoresis}

As with HPLC, a wide variety of detector types and detection schemes are available for CE. Laser induced fluorescence (LIF), laser induced native fluorescence (LINF), UV-Vis, mass spectrometry, and electrochemical detection have all been successfully used in conjunction with capillary electrophoresis. In commercially available instrumentation, detection is still overwhelmingly LIF and UV-Vis based, with MS 
detectors only recently becoming commercially available. Electrochemical detectors remain most popular in commercial HPLC instruments for neurotransmitter analysis, with most applications of electrochemical detection in conjunction with $\mathrm{CE}$ being done on homebuilt systems.

A.

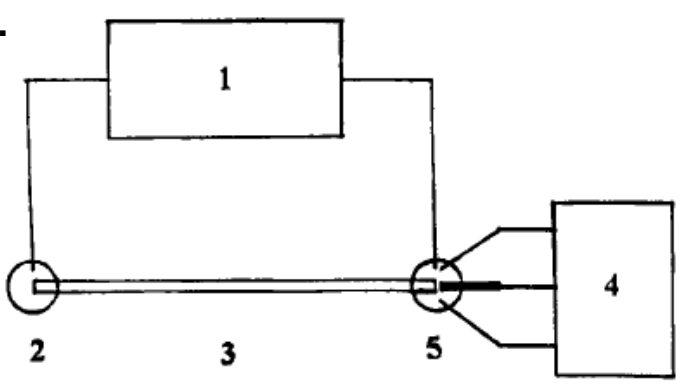

B.

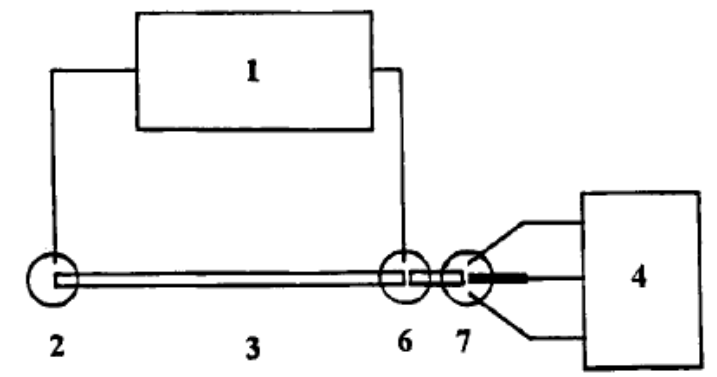

Figure 1-7: Common electrochemical detector formats for capillary electrophoresis. A. Endcolumn configuration B. off-column configuration. 1. High voltage power supply 2. Buffer reservoir 3. Fused silica capillary 4. Potentiostat 5 . Cell for end column electrochemical detection 6. CE anode reservoir for decoupled system 7 . Cell for decoupled electrochemical detection. Reprinted (adapted) with permission, from Voegel et al. ${ }^{79}$
A variety of factors make the use and installation of electrochemical detectors complicated. In HPLC systems, since flow is pressure driven (as opposed to electric field driven as in $\mathrm{CE})$, electrochemical detection is relatively simple; working electrode, auxiliary, and reference electrodes are placed at the end of the column. In capillary electrophoresis, the high applied separation voltage may interfere with the low detection voltage. ${ }^{74}$ This interference shifts the applied electrode potential to more positive potentials. ${ }^{75}$ As a result, decoupling of separation voltage to avoid interference with applied potentials at the surface of the electrode is desirable. ${ }^{76-78}$ Decoupling can either be done before the detector (oncapillary, which creates a separate detection capillary) or at the end of the capillary, which has led to two common detector configurations for CE-EC systems: end-column (Figure $9 \mathrm{~A}$ ) and off-column (Figure $9 \mathrm{~B}){ }^{79}$

\subsubsection{Decoupling for Electrochemical Detection in CE}


Decoupling can be accomplished in a variety of ways, with some methods being more complicated than others. Generally, decouplers can be classed as on-column or

A.

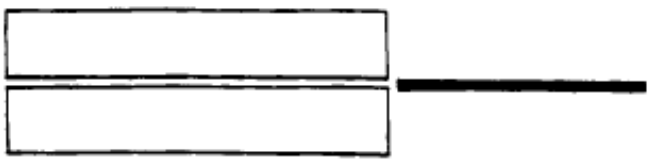

B.

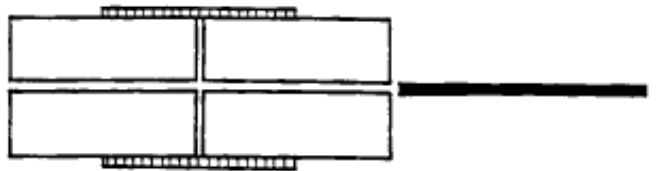

C.

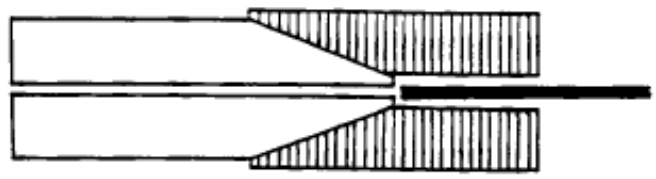

D.

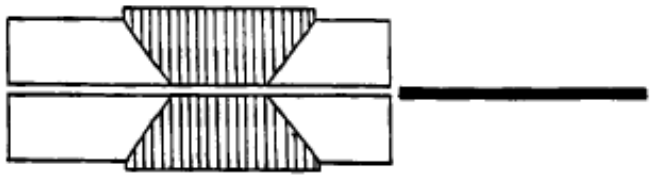

Figure 1-8: Decoupling methods for CE-EC:

A. End column (no decoupling), B. covered fracture (nafion or cellulose acetate), C. cast Nafion endcolumn decoupler, and $\mathrm{D}$. Long case cellulose acetate or Nafion decoupler. The shaded areas represent the decoupler. Adapted with permission, from Voegel et al. $^{79}$ end-column. End column decoupling can

be done using metal decouplers, which

are positioned between the end of the capillary and the detector, added Nafion endings (Figure $10 \mathrm{C}$ ) or etched sections of capillary near the end. ${ }^{78,80,81}$ End column applications way also use no decoupler in certain circumstances (Figure 10A). ${ }^{78}$ On-column decoupling is more complicated and can be performed by creating porous junctions or fractured sections of the capillary which are covered in sleeves (Nafion or cellulose acetate) (Figure $10 \mathrm{~B}, \mathrm{D}){ }^{82,83}$ On-column

decouplers are absolutely necessary for on-column/on-capillary detection. Fracture decouplers are immersed in buffer solution and grounded. The major drawback of this type of decoupler is the low reproducibility and increased diffusional band broadening, which results in decreased sensitivity. For all methods of decoupling, there is some sacrifice of robustness of the CE setup and possible band broadening. 
It is possible to avoid decoupling altogether under certain circumstances. As

previously mentioned, low inner

diameter capillaries are used to

increase the voltage drop across the

length of the capillary ${ }^{84}$. The high

voltage drop is due to the high

resistance of these small bore

capillaries. This greatly reduces the

separation voltage so that at the end

of the capillary, where the detector is

positioned, it no longer (or minimally)

interferes with detection. Alignment of

the working electrode with the capillary

end is critical with this approach. The

working electrode can be positioned

within a capillary diameter away from (a)

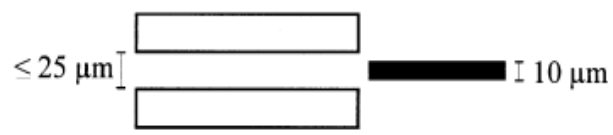

(b)

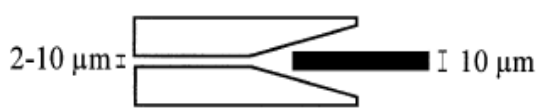

(c) $50-75 \mu \mathrm{m}$
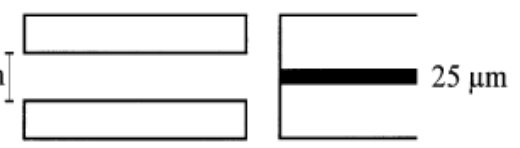

(d)

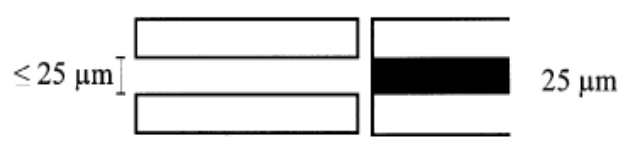

(e)

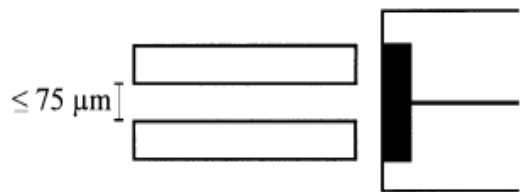

Figure 1-9:Typical capillary-to-electrode alignments used for CE-EC without decouplers. a) Carbon fiber or metal electrodes positioned close to the capillary outlet, b) fiber electrode placed in a conically etched capillary end, c-e) disk electrodes with diameters smaller, equal or larger than capillary diameter. Reprinted (adapted) with permission, from Matysik. ${ }^{78}$

the capillary outlet. ${ }^{81}$ Additionally, this approach is poorly suited to fiber (or cylinder) electrodes (Figure $10 \mathrm{~A}$ ), as the electroactive surface of the sensor does not come in complete contact with eluting solute zones. These electrodes are best used in etched ending capillaries where the electrode may be positioned inside of the etched end (Figure $10 \mathrm{~B}$ ), however there may be interference from the applied separation voltage. Disk electrodes are preferable in these arrangements due to the trapezoidal flow profile of elecroosmotically driven flow (Figure 10C-E). Disk electrodes should either have a working surface that is the same or greater diameter as the capillary inner diameter for similar reasons. Alignment is often done using specialized flow cells with guides, and 
micromanipulators to ensure alignment in $\mathrm{x}, \mathrm{y}$ and $\mathrm{z}$ planes, although other innovative approaches to alignment exist, such as customized flow cells. ${ }^{80}$

Some of the issues with decoupling have been either resolved or minimized with the rise of capillary electrophoresis on chip. ${ }^{85}$ Microfluidic platforms allow for integration of decouplers and can allow for simpler alignment of detector or the fabrication of detector arrays. ${ }^{86,87}$ The principles of electrophoresis on chip are similar to those of traditional CE, but the smaller form factor and materials used for the chips and detectors differ from those used in capillary electrophoresis coupled to electrochemical detection (CE-EC) setups. Instead of fused silica, channels in chips are made from polymers or glass, and can be assembled in a wide variety of ways. ${ }^{86,88,89} \mathrm{CE}$ on a chip also can handle small sample volumes just as CE, but uses smaller applied separation voltages, and can be built to accommodate a variety of sample injection formats. ${ }^{90,91}$ The small footprint leads to fast separations. For CE-EC on chip, amperometry is the most common detector due to its extremely high sensitivity, but other modes of electrochemical detection have also been developed such as contactless conductivity and voltammetric methods. ${ }^{88,92}$ Lab on a chip based systems for CE and neurotransmitter analysis is an active area of research. These systems would enable sample collection and analysis from awake and moving animals. ${ }^{93}$

\subsubsection{Electrochemical Detection in Capillary Electrophoresis}

\subsubsection{Amperometry}

For chip and CE separations using electrochemical detection, amperometry has been widely used. Amperometry provides high sensitivity and is widely documented for the detection of neurotransmitters ${ }^{64,65,81,92}$. This high sensitivity is due to the application of a constant potential sufficient to oxidize analytes of interest applied to the working electrode. A wide variety of working electrode materials are used, but carbon fiber 
microelectrodes are among the most popular due to their resistance to fouling and wide operating range ${ }^{94-96}$. Metal electrodes, such as gold, are used as well, but are limited by small potential ranges which increase the risk of interference from oxidation of water ${ }^{87}$. Amperometric sensors produce current over time data, with response times reaching the sub millisecond scale in certain cases, providing high time resolution ${ }^{42}$. This high time resolution in conjunction with separatory methods allows for quick separations of extremely dilute samples. However, as with many other detector types, amperometry provides little identifying information on the analyte and peaks must be identified based on separations of standards alone. Amperometry makes identification of unknown analytes in biological samples more challenging and can reduce confidence in peak identity when working with challenging samples. Peak misidentification using amperometry has been well documented by Hirsh and coworkers using $D$. melanogaster samples analyzed by HPLC coupled to amperometry. ${ }^{41}$ Despite the differences in the separation method, these lessons are still applicable to amperometry coupled to capillary electrophoresis.

CE-Amperometry was first reported by Ewing and co-workers for the detection of neurotransmitters. ${ }^{65,97}$ Initially, CE-amperometry was used for the analysis of cells from Planorbis corneus. ${ }^{98}$ Later applications focused on the determination of neurotransmitters in the adult brain of Drosophila, as well as determination of the presence of metabolites of neurotransmitters in these tissues. ${ }^{94,95,99}$ Ewing and coworkers also performed microdissections of the adult brain to determine region specificity of neurotransmitters. ${ }^{95}$ Some on-chip applications of amperometry for the detection of neurotransmitters have been reported by Lunte and co-workers. ${ }^{86,100}$

\subsubsection{Fast Scan Cyclic Voltammetry}


Fast scan cyclic voltammetry is another electrochemical method used for detection of neurotransmitters coupled to CE., In cyclic voltammery, the applied potential is varied over time. A typical waveform used in cyclic voltammetry (and fast scan cyclic voltammetry) is the triangular waveform (Figure 12).

In this waveform, the potential of the electrode is ramped linearly versus

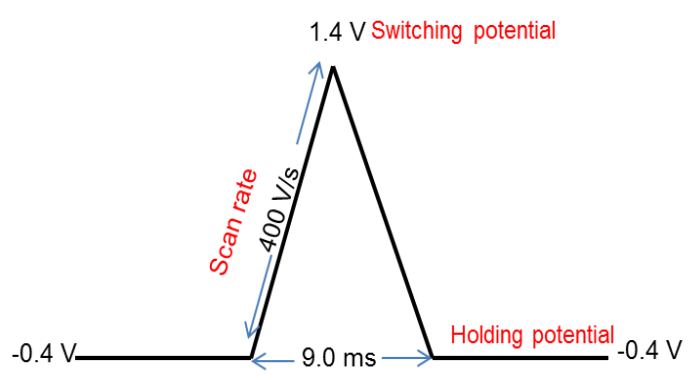

Figure 1-10: A triangular waveform as used in FSCV.

The holding potential $(-0.4 \mathrm{~V})$ is scanned up to the switching potential at a set scan rate (here, $400 \mathrm{~V} / \mathrm{s}$ ). After reaching the switching potential $(1.4 \mathrm{~V})$, the applied voltage is ramped back down to the holding potential. In the example above, this ramp up and down takes less than $100 \mathrm{~ms}$. In traditional CV, scan rates are much lower $(10 \mathrm{mV} / \mathrm{s})$ and as a result the scans take longer.

time from the holding potential until it reaches a switching potential. At the switching potential, the potential is then ramped down again back to the holding potential. Other

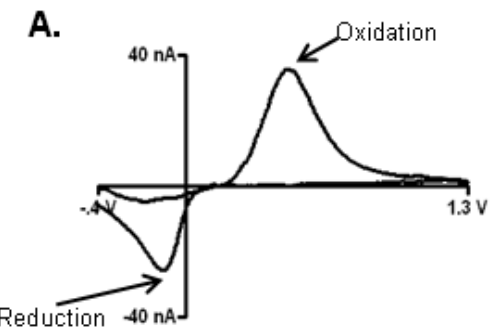

B. Dopamine Dopamine-o-quinone

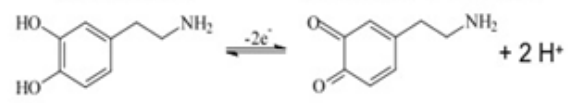

Figure 1-11: Cyclic voltammetry of Dopamine.

A. A cyclic voltammogram of dopamine obtained using fast scan cyclic voltammetry. The anodic (oxidation) and cathodic (reduction) peaks are visible at $\sim .6 \mathrm{~V}$ and $\sim-0.1 \mathrm{~V}$ respectively. This cyclic voltammogram can be used for quantitation and analyte identification, as the peaks are characteristic of the redox properties of the analyte. B. The oxidation and reduction reaction of dopamine: dopamine is oxidized to dopamine-oquinone, and dopamine-o-quninone is reduced back to dopamine. variations on these scanning parameters exist, but triangular waveforms are the most common.

During the scan, the current is measured and a current vs. voltage plot is generated, known as a cyclic voltammogram (Figure $13 \mathrm{~A}$ ). The first ramp, from holding to switching potential, causes oxidation of the analyte (if the applied oxidation potential is sufficient). This generates a characteristic oxidation peak (anodic peak) for the analyte. If the oxidiation of the analyte is reversible, then on the back scan a reduction peak (cathodic peak) will be produced (Figure $13 \mathrm{~B})$. The anodic and cathodic peaks provide 
information about the identity of the analyte as well as its redox properties. Due to scanning, time resolution and sensitivity can suffer, especially in traditional cyclic voltammetry, where scan rates are low (under $100 \mathrm{mV} / \mathrm{s}$ ). Fast scan cyclic voltammetry improves upon some of these limitations by increasing the scan rate to $100 \mathrm{~V} / \mathrm{s}$ or higher, with typical scan rates around $400 \mathrm{~V} / \mathrm{s}$.

High scan rates drastically increase both sensitivity and time response, with millisecond time response and low $\mathrm{nM}$ limits of detection possible for analytes like dopamine. Analytes that undergo irreversible oxidations may also be analyzed by cyclic voltammetry as well, permitting analysis of analytes such as adenosine. With FSCV the higher scan rates and scan frequency produce a series of cyclic voltammograms, which can be depicted as a false color plot (Figure 14). This false color plot shows changes in

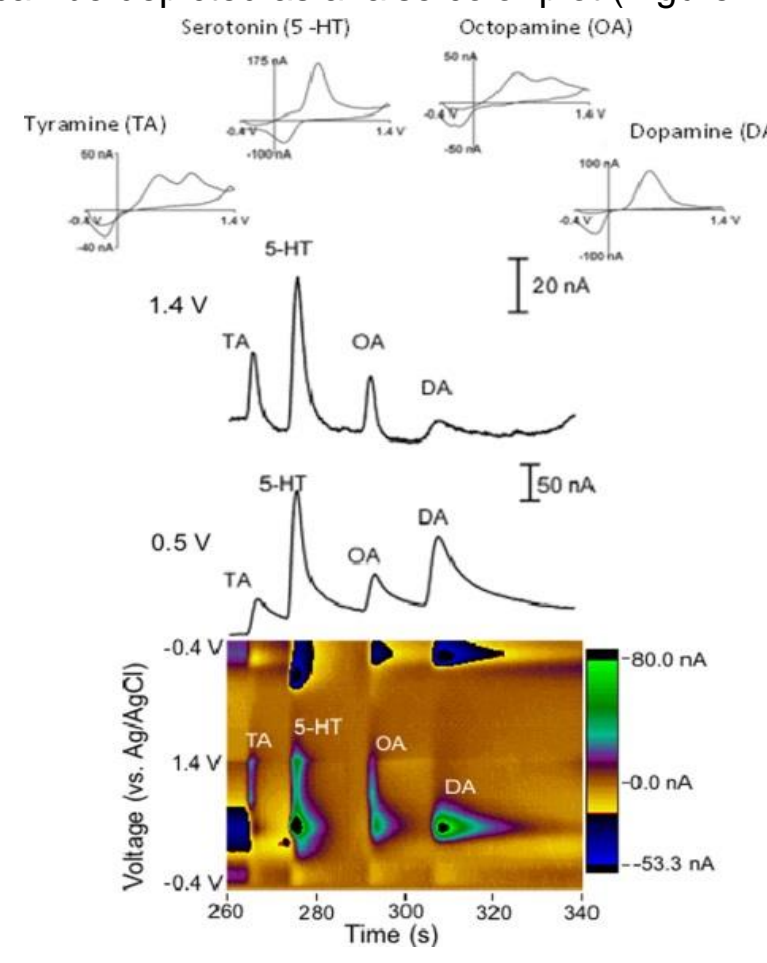

Figure 1-12: Capillary electrophoresis with Fast Scan Cyclic Voltammetry.

Data from a CE-FSCV set up showing cyclic voltammograms (top), current vs. time traces (middle), and false color plot (bottom). Reprinted (adapted) with permission, from Denno et. al. ${ }^{11}$ current (oxidation in green, reduction in

blue). A current vs. time plot can also be generated (at a given voltage) to show the change in current at an applied potential. In Figure 14, the limited utility of these current vs. time traces in a separation are seen as not all analytes are visible at all potentials. Furthermore, in contrast to amperometric methods, quantitation cannot be done from these peaks. However, in conjunction with the color plot and cyclic voltammograms, the current vs. time traces provide information on the separation and peak 
identity. With a separation method, these three pieces of information provide an electropherogram (the current vs. time trace at a given potential), a peak identification both by the electropherogram and cyclic voltammogram, and a "map" of all current vs. time traces at any potential (the color plot). The variety and amount of information available through this combination of CE and FSCV increases confidence in the separation.

\subsubsection{Application of CE-FSCV to Separation and Detection of Neurotransmitters In our lab, this combination has been} used to study the neurotransmitter content of tissue from the central nervous system of $D$. melanogaster. Early applications focused on the $3^{\text {rd }}$ instar larva, which our lab has investigated as
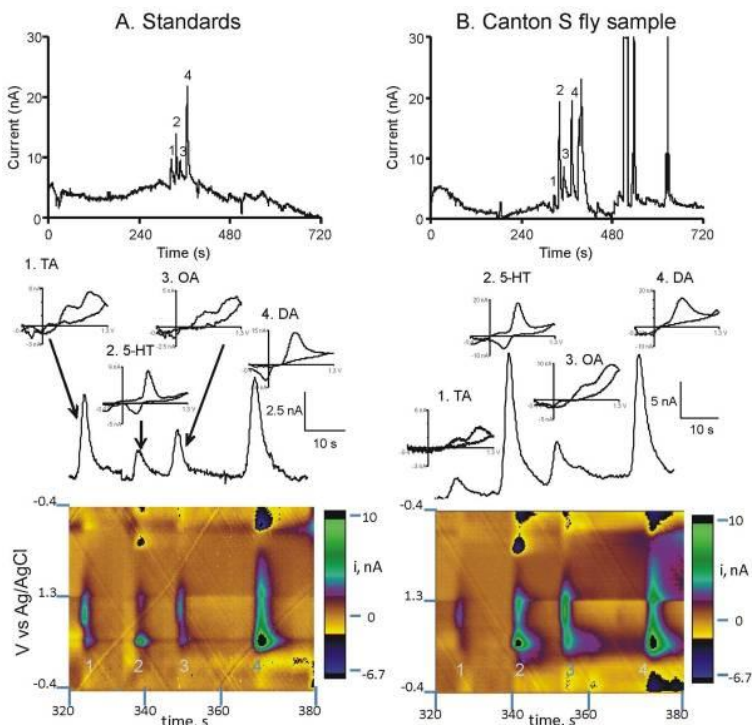

Figure 1-14: Separations of standards and fly tissue by CE-FSCV.

A. Separation of a standard sample $(50 \mathrm{nM}$ tyramine (TA), octopamine (OA), dopamine (DA) and $25 \mathrm{nM}$ serotonin (5-HT)). B. Separation of single Canton S VNC, diluted in acetonitrile and perchloric acid. (16 nM tyramine, $51 \mathrm{nM}$ serotonin, $111 \mathrm{nM}$ octopamine, and 64 nM dopamine.) Reprinted (adapted) with permission from Fang et al. 2011. ${ }^{101}$

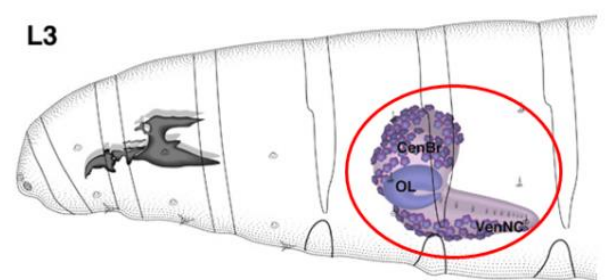

Figure 1-13: The larval Drosophila central nervous system.

The central nervous system is circled in red, and is approximate $8 \mathrm{~nL}$ in volume. Adapted with permission from Atlas of Drosophila Development. ${ }^{116}$

a model for kinetics of neurotransmitter clearance and metabolism. ${ }^{25}$ The intact central nervous system (ventral nerve cord (VNC)) of a $3^{\text {rd }}$ instar larva is estimated to be $8 \mathrm{~nL}$, and as such, analysis of single VNCs requires high sensitivity and the ability to handle small samples (Figure 15). VNC samples could be homogenized, diluted, and injected for analysis of four neurotransmitters: tyramine (TA), serotonin $(5-H T)$, octopamine $(\mathrm{OA})$, 
and dopamine (DA) (Figure 16). ${ }^{101}$ Multiple wild type flies were compared, as well as drug treated, and mutant flies. Tyramine quantitation was not possible in all samples due to its low concentration in the VNC. In several cases, these were the first recorded tissue content values for larva available in these strains. Additionally, CE-FSCV was able to detect and quantify neurotransmitters from a single VNC; other previously reported values could require as many as 25 flies. The ability to analyze single flies demonstrated the power of CE-FSCV for analysis of Drosophila samples that were traditionally done using HPLC.

In addition to application to Drosopila samples, CE-FSCV was also used for determination of tissue content of neurotransmitters from tissue punches obtained from rat brain slices. ${ }^{10}$ Tissue punches from various brain regions were homogenized and diluted in a small volume of perchloric acid, and then were injected. Using this method, dopamine, serotonin, and adenosine were detected from a variety of small $(400 \mu \mathrm{m}$ thick to $100 \mu \mathrm{m}$ thick) punches obtained

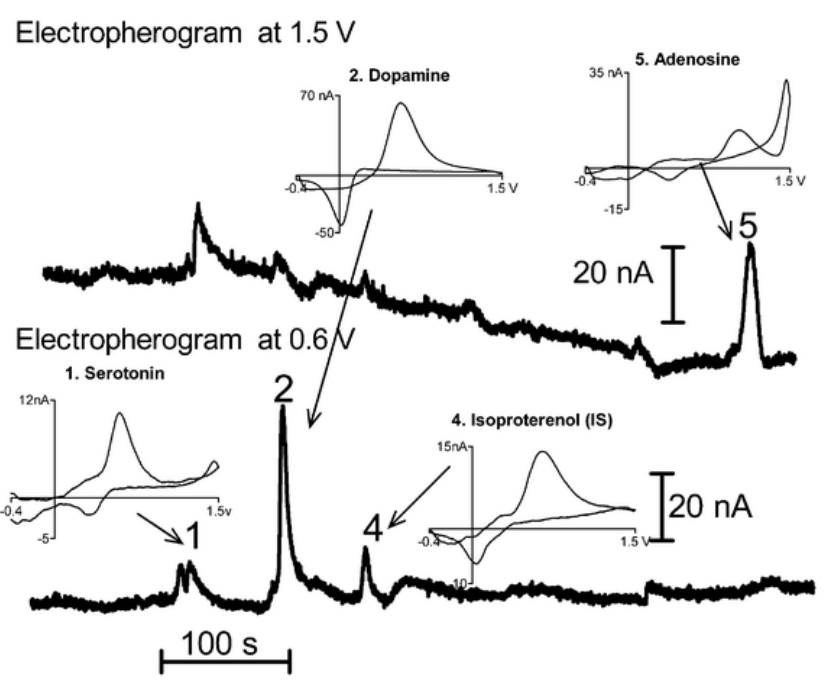

Figure 1-15:Separations of neurotransmitters found in rat brain tissue punches.

Tissue content of a $100 \mu \mathrm{m}$ thick slice (300 $\mu \mathrm{m}$ diameter) from the frontal cortex. Serotonin, dopamine, and adenosine are clearly detected in this small sample; isoproterenol is the internal standard. The amount of analyte in this tissue punch is $0.082 \mathrm{pmol}$ of serotonin, $0.12 \mathrm{pmol}$ of dopamine, and $4.3 \mathrm{pmol}$ of adenosine. Reprinted (adapted) with permission, from Fang et. Al. 2013. ${ }^{10}$

from the caudate putamen of the rat; all analytes of interest were quantifiable even in the smallest tissue punch obtained (Figure 17). Tissue punches from sequential slices were also examined to determine the varriation in tissue content from the front of the region 
to the back of the region. In addition to these tissue content determinations of a variety of different neurotransmitters, CE-FSCV was used to correlate tissue content to stimulated release. A brain slice was stimulated to release dopamine, the concentration of dopamine released was recorded by FSCV, and then that region was removed using a tissue punch. The tissue was then processed, and analyzed using CE-FSCV to determine its local concentration. There was a strong correlation between dopamine concentration released due to simulated release and tissue content $(R=0.7)$.

\subsection{Challenges for CE-FSCV Application to Drosophila}

\subsubsection{Drosophila as a Model Organism: Moving on from Larva, Tracking} Development

While we have previously used

CE-FSCV to determine the

neurotransmitter tissue content of larval ventral nerve cords and we typically work with larval ventral nerve cords to determine metabolism and kinetics of neurotransmitters, the vast majority of work using Drosophila as a model organism has focused on adults. As such, understanding the differences between the larval and the adult brain, as well as the impact of metamorphosis on the tissue content of the brain is

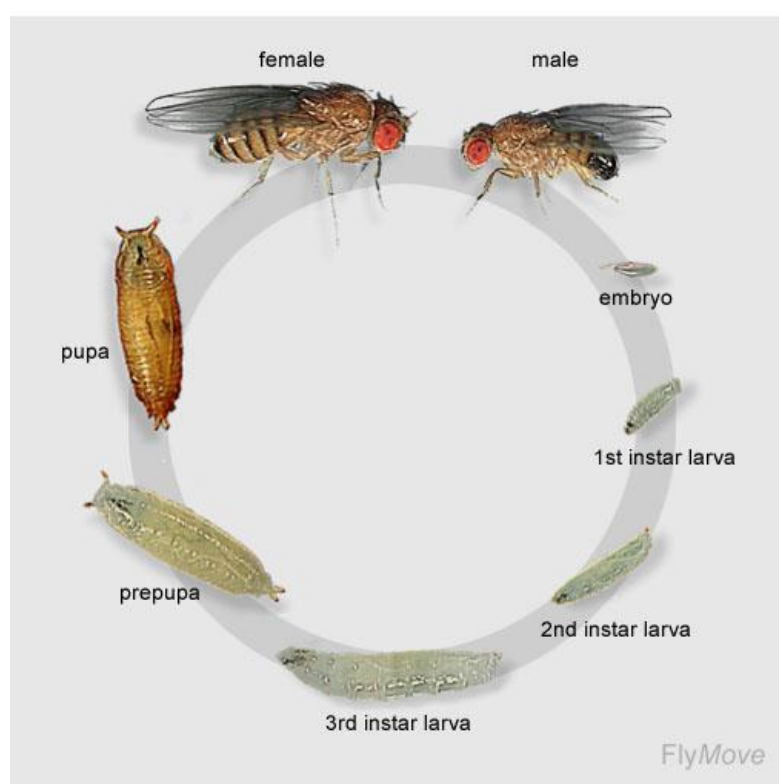

Figure 1-16: Life cycle of Drosophila melanogaster.

After egg laying, the embryo develops and hatches into a $1^{\text {st }}$ instar larva in a day. After that, the larva goes through two more instars, with the $3^{\text {rd }}$ instar being the largest and latest. The $3^{\text {rd }}$ instar larva then attached itself to a wall or elevated surface and ecloses within 3 days. Reprinted (adapted) with permission, from FlyMove. ${ }^{127}$ important.

\subsubsection{Drosophila development}


Drosophila is holometabolous, meaning that it undergoes metamorphosis between four distinct life stages (Figure 16): the embryo, the larva, the pupa, and the adult. The brain is present in a rudimentary form late in the embryonic stage (Figure 17) and develops throughout the larval stage, growing larger during each instar. After the third instar larva stage, the larva pupates. During pupation, significant arborization and

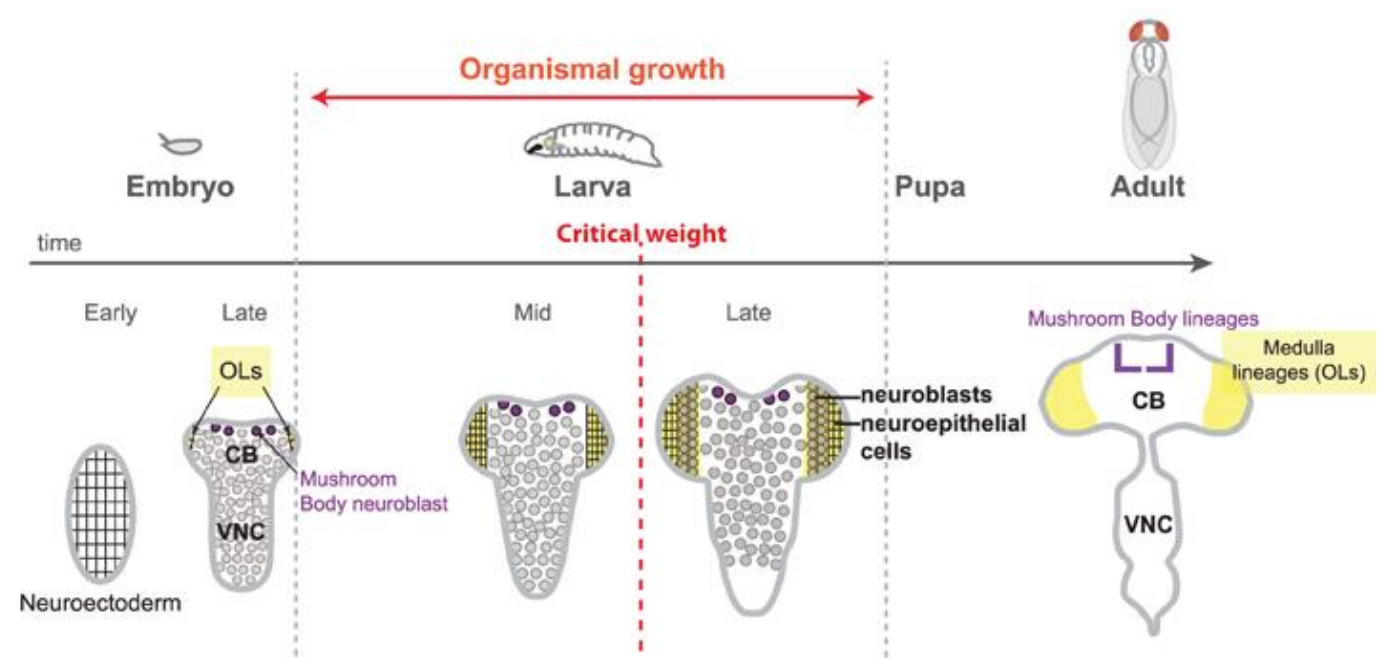

Figure 1-17: The central nervous system of Drosophila melanogaster changes during development.

As Drosophila undergoes metamorphisis, the central nervous system grows, develops and changes. The basic footprint of the brain emerges in the late embryonic stage, with further development in size and shape occurring during the larval period as the larva eats and grows. During pupation (not shown) there is significant reorganization. Post eclosion, the adult brain and its structures are formed and functional. Reprinted (adapted) with permission, from Lanet et al. ${ }^{128}$

rearrangement of the brain occurs, and the structures found in the adult brain (such as

the mushroom bodies) develop. Additionally, connections from the central nervous

system to different segments of the body are rewired, changing the way the brain

communicates with the muscles. During this stage, the pupa is immobile and remains so until the adult fly ecloses from the pupa. Post eclosion, the brain is in its adult form.

\subsubsection{Comparisons between Life Stages}

We have previously reported tissue content of single larval VNCs in wild type strains of Drosophila. ${ }^{101}$ Comparison of drug treated and untreated larval VNCs was also performed, which allowed for determination of the effects of drug consumption on the 
tissue content of larval brains. Adult brains have previously been analyzed by other groups, typically by HPLC using large pooled samples, and have been used to study aging and the impact of age on tissue neurotransmitter content. ${ }^{102}$ Analysis of adult heads and brains to determine the difference in neurotransmitter content between strains of mutant flies has also been done. ${ }^{41,103}$ While the central nervous systems of both stages are different, both are commonly used to understand neurotransmitter release and clearance.

While both the larval central nervous system and the adult brain have been studied in the past, the tissue content of the pupal brain for any strain of fly has not previously been reported. This is likely due to the fact that the pupa is relatively uninteresting: it is immobile, cannot perform tasks, and is more interesting for the understanding of limb development from imaginal discs. However, the large amount of development occurring in the central nervous system as well as the immobile nature of the pupa raises some interesting questions about neurotransmitter tissue content. The neurotransmitter octopamine play a large role in locomotion, feeding, and learning for Drosophila. Octopamine is important in the larva and the adult, but the role and presence of different neurotransmitters in pupa may be drastically different due to its development and sessile nature. Determining the neurotransmitter content of the pupa may be of interest in understanding what changes are occurring in gene expression in the brain, and how or if these changes persist in the adult or if the pupal brain is more similar to the larval brain.

Comparison of the adult and larval brain tissue content of a variety of neurotransmitters is important to help understand the differences that may arise in stimulated release work in larval central nervous system and the adult brain. If tissue content is comparable and stimulated release values are not, this may help to show that 
those differences are not due to releasable pools or total neurotransmitter present, but rather expression of an optogenetic channel or localization of neurotransmitter to a specific area in the tissue. Understanding the differences in tissue content between life stages can indicate changes to synthesis or metabolism of neurotransmitters. This may also provide better ways of analyzing fly models of neurodegenerative diseases. Models of neurodegenerative disease may currently analyzed throughout the larval stage or throughout their adult stage, but it is possible that there may be value in comparing larval and adult neurotransmitter tissue content to determine the progression of disease or the onset of disease. Additionally, these comparisons may elucidate if larva and adults may be used as complementary models for human disease.

Lastly, the majority of neurotransmitter tissue content analysis done in Drosophila by other groups has been done in adults, and as such, looking soley at larva limits the scope and usefulness of our technique for other researchers considerably. As Drosophila undergoes significant changes in morphology throughout its life cycle (Figure 18), there may be appreciable differences in the neurotransmitter content of adults and larva due to changes in gene expression and regulation as the fly develops. For a more complete understanding of neurotransmitters in the brain and central nervous system, multiple stages of development in Drosophila must be compared. Tissue content from adult flies would likely be more broadly useful, especially when coupled with studies of neurotransmitter clearance and uptake kinetics in adult flies.

\subsubsection{Separations of Non-Catecholamine and Phenolamine Neurotransmitters and their Metabolites \\ Previous work using CE-FSCV focused on the separation and detection of} catecholamine and phenolamine compounds. Capillary electrophoresis separation methods for catecholamines are widely available, and our previous experience with them gave separation conditions that were appropriate. As there are a variety of other 
important neurotransmitters and metabolites that are present in fly tissues, expansion of our separation conditions and methods to detect and quantify these compounds is important.

\subsubsection{Histamine, Carcinine, and $N$ - $\beta$-alanyl Dopamine}

Histamine, which is a

neurotransmitter found both in mammals

and in Drosophila, is therefore an

attractive target for investigation. Fast

scan cyclic voltammetric detection of

histamine has been reported for in vivo

measurements in rats and zebra fish,

another popular model organism. ${ }^{104-106} \mathrm{~A}$

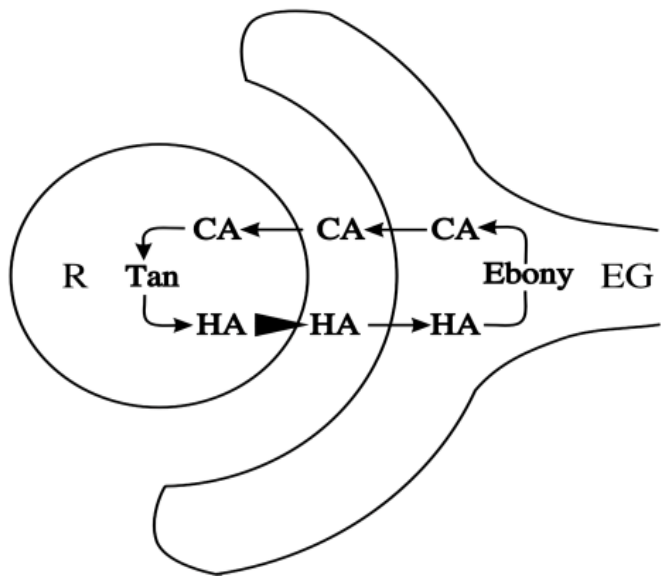

previously discussed, histamine plays an

important role in Drosophila vision,

peripheral nervous system sensing

through hairs and circadian rhythm

maintenance. ${ }^{21,107}$ Tracking histamine and

its metabolism in Drosophila is therefore

of value due to the critical nature of these

functions. Furthermore, better

understanding of histamine may lead to

improved understanding of the role of

histamine in human neurological diseases

and disorders. ${ }^{108}$

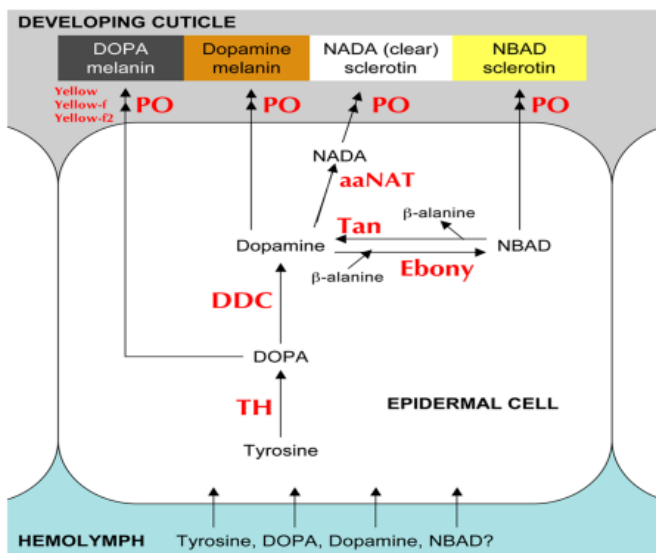

Figure 1-18: The role of Ebony and Tan in the metabolism of dopamine and histamine.

A) In the photoreceptor of Drosophila, Tan catalyzes the hydrolysis of $\mathrm{N}-\beta$-alanyl histamine (carcinine) to histamine so that it can undergo reuptake by the presynaptic photoreceptor cell. B) In adult epidermal cells, Tan catalyzes the production of dopamine from NBAD during pigmentation development. This is one of several parallel pathways through which dopamine and dopa derivatives are used as pigment precursors. Reprinted (adapted) with permission from Liou at al. 2005. ${ }^{121}$

Previous attempts to determine 
the tissue content of histamine in Drosophila were done using HPLC, and required large pooled samples of whole heads and pretreatment of the sample for amperometric detection. ${ }^{109,110}$ Furthermore, monitoring of the primary metabolite of histamine in Drosophila, carcinine, was not possible using this method. This is likely due to low abundance of carcinine and its electrochemical properties; $\beta$-alanylated compounds are less electroactive and are therefore difficult to detect. Monitoring of carcinine using radiolabeling was performed, but this loses important information on the tissue content of the metabolite. Additionally, increased tissue specificity is required to understand histamine and carcinine metabolism: whole head samples do not allow for determination of brain and eye tissue contents. As the eye contain high levels of these compounds, separation into cuticle, eye, and brain is necessary to understand the histamine cycle more accurately.

Histamine is metabolized to carcinine by $\mathrm{N}$ - $\beta$-alanyl dopamine synthetase (also called Ebony), and carcinine is recycled back to histamine by $\mathrm{N}$ - $\beta$-alanyl dopamine hydrolase (Tan) (Figure 18A). Mutants that have reduced or abolished activity of these enzymes are available, which allows comparison with wild type metabolism. Ebony and Tan are important for multiple processes. In the visual system, recycling of histamine to carcinine is required for normal vision and visual processing, and large amounts of both are present in the eyes. ${ }^{110,111}$ Changes in histamine metabolism in Drosophila appear to cause behavioral changes as well, some of which may not be directly attributable to visual defficits. ${ }^{112}$ There are only a small number of histamine neurons present in the adult Drosophila brain, but they appear to perform important functions. ${ }^{113}$

In addition to histamine, Ebony metabolizes dopamine to $\mathrm{N}$ - $\beta$-alanyl dopamine (BADA) and Tan metabolizes BADA to dopamine. As Drosophila lacks a monoamine oxidase, metabolism of dopamine in the brain is still poorly understood. This gap makes 
the study of the dopamine and BADA cycle in selected tissues valuable. BADA has been documented to be important for pigmentation of cuticle and the cross linking of cuticular tissues in Drosophila, but has no known role in the brain (Figure 18 B). ${ }^{112,114,115}$ While BADA's primary importance appears to be in the development and coloration of tissue, Ebony and Tan are expressed in the brain. Expression of these genes in the brain may be linked not only to histamine metabolism, but also dopamine metabolism, and previous studies have not been able to determine if this occurs.

\subsubsection{The Role of Vision, Eye Coloration, and Development on Histamine and Carcinine}

Changes to other aspects of the visual system, while not necessarily caused by mutations in the histamine metabolic cycle, may also alter the tissue content of histamine in the brain and eyes. Mutants without eyes, or with abnormal eye function or formation may have reduced histamine content, and therefore reduced carcinine content. ${ }^{109}$ Previous attempts to quantify histamine in the heads of sine oculis flies showed a reduced content of histamine in whole heads relative to wild type flies. Fly heads with non-wild type eye colors (white, brown, and scarlet) have also previously been shown to have reduced histamine content. ${ }^{103}$ Given the large number of other genes involved in the development of eyes (such as eyeless and eyes absent) and the proper formation of the compound eye surface (such as glass), it is of importance to understand if there is a knock-on effect of defective eye development on brain and eye tissue content of histamine and carcinine.

While the impact of these mutations is obvious in adults, there is little understanding of if these mutations impact the histamine and carcinine content of the larval central nervous system. While the larva lacks compound eyes and instead "sees" using the Bolwig organ, tissues that will develop into the adult eyes are present in the form of imaginal discs. ${ }^{19,116}$ In addition to this, it is unclear what kind of histamine content 
the larval brain may have and how its content of histamine compares to that of the adult. Determining the histamine content of the larva in both wild type and mutant flies can complement adult tissue content information as well as provide information on if the metabolic profile of histamine and carcinine change with development, and if these eye development mutations already impact the brain before they are obvious.

Overall, expansion of CE-FSCV to the study of histamine, carcinine, and BADA would enable direct study of histamine and carcinine without the need for isotopic tagging and allow for greater tissue specificity in the analysis of Drosophila. In addition, the ability to detect BADA would provide answers on if this metabolite is important for the metabolism of dopamine in the brain. The expansion of histamine determination to a wide variety of eye and eye development mutants allows for better understanding of the brain histamine circuit and the impact of visual input on these circuits. Lastly, tracking of neurotransmitters over the course of development can help to see when these mutations begin to alter the metabolism or production of histamine and carcinine. 


\subsection{References}

(1) Findley, L., Aujla, M., Bain, P. G., Baker, M., Beech, C., Bowman, C., Holmes, J., Kingdom, W. K., MacMahon, D. G., Peto, V., and Playfer, J. R. Direct economic impact of Parkinson's disease: A research survey in the United Kingdom. Mov. Disord. Wiley Subscription Services, Inc., A Wiley Company.

(2) Mitkovski, M., Padovan-Neto, F. E., Raisman-Vozari, R., Ginestet, L., da-Silva, C. A., and Del-Bel, E. A. (2012) Investigations into Potential Extrasynaptic Communication between the Dopaminergic and Nitrergic Systems. Front. Physiol. 3, 372.

(3) Zoli, M. (1999) Volume transmission in the CNS and its relevance for neuropsychopharmacology. Trends Pharmacol. Sci. 20, 142-150.

(4) Syková, E. (2004) Extrasynaptic volume transmission and diffusion parameters of the extracellular space. Neuroscience 129, 861-76.

(5) Kato, G., Yasaka, T., Katafuchi, T., Furue, H., Mizuno, M., Iwamoto, Y., and Yoshimura, M. (2006) Direct GABAergic and glycinergic inhibition of the substantia gelatinosa from the rostral ventromedial medulla revealed by in vivo patch-clamp analysis in rats. J. Neurosci. 26, 1787-94.

(6) Mansor, M. A., and Ahmad, M. R. (2015) Single Cell Electrical Characterization Techniques. Int. J. Mol. Sci. 16, 12686-12712.

(7) Colliver, T. L., Pyott, S. J., Achalabun, M., and Ewing, A. G. (2000) VMAT-Mediated Changes in Quantal Size and Vesicular Volume. J. Neurosci. 20, 5276-5282.

(8) Omiatek, D. M., Dong, Y., Heien, M. L., and Ewing, A. G. (2010) Only a Fraction of Quantal Content is Released During Exocytosis as Revealed by Electrochemical Cytometry of Secretory Vesicles. ACS Chem. Neurosci. 1, 234-245.

(9) Watson, C. J., Venton, B. J., and Kennedy, R. T. (2006) In vivo measurements of neurotransmitters by microdialysis sampling. Anal. Chem. 78, 1391-9.

(10) Fang, H., Pajski, M. L., Ross, A. E., and Venton, B. J. (2013) Quantitation of dopamine, serotonin and adenosine content in a tissue punch from a brain slice using capillary electrophoresis with fast-scan cyclic voltammetry detection. Anal. Methods. The Royal Society of Chemistry.

(11) Denno, M. E., Privman, E., and Venton, B. J. (2015) Analysis of neurotransmitter tissue content of Drosophila melanogaster in different life stages. ACS Chem. Neurosci. $6,117-23$.

(12) lannaccone, P. M., and Jacob, H. J. Rats! Dis. Model. Mech. 2, 206-10.

(13) Abbott, A. (2004) Laboratory animals: the Renaissance rat. Nature 428, 464-6.

(14) Baier, A., Wittek, B., and Brembs, B. (2002) Drosophila as a new model organism for the neurobiology of aggression? J. Exp. Biol. 205, 1233-1240.

(15) Reiter, L. T., Potocki, L., Chien, S., Gribskov, M., and Bier, E. (2001) A systematic analysis of human disease-associated gene sequences in Drosophila melanogaster. Genome Res. 11, 1114-25.

(16) Adams, M. D. (2000) The Genome Sequence of Drosophila melanogaster. Science (80-. ). 287, 2185-2195. 
(17) Reiter, L. T., Potocki, L., Chien, S., Gribskov, M., and Bier, E. (2001) A systematic analysis of human disease-associated gene sequences in Drosophila melanogaster. Genome Res. 11, 1114-25.

(18) Nichols, C. D. (2006) Drosophila melanogaster neurobiology, neuropharmacology, and how the fly can inform central nervous system drug discovery. Pharmacol. Ther. 112, 677-700.

(19) Martin, C. A., and Krantz, D. E. (2014) Drosophila melanogaster as a genetic model system to study neurotransmitter transporters. Neurochem. Int. 73, 71-88.

(20) Haas, H. L., Sergeeva, O. A., and Selbach, O. (2008) Histamine in the nervous system. Physiol. Rev. 88, 1183-241.

(21) Nitabach, M. N., and Taghert, P. H. (2008) Organization of the Drosophila circadian control circuit. Curr. Biol. 18, R84-93.

(22) Roeder, T. (2005) Tyramine and Octopamine: Ruling Behavior and Metabolism. Annu. Rev. Entomol. 50, 447-477.

(23) Fenno, L., Yizhar, O., and Deisseroth, K. (2011) The development and application of optogenetics. Annu. Rev. Neurosci. 34, 389-412.

(24) Kravitz, A. V, and Kreitzer, A. C. (2011) Optogenetic manipulation of neural circuitry in vivo. Curr. Opin. Neurobiol. 21, 433-9.

(25) Vickrey, T. L., Condron, B., and Venton, B. J. J. (2009) Detection of Endogenous Dopamine Changes in Drosophila melanogaster Using Fast-Scan Cyclic Voltammetry. Anal. Chem. 81, 9306-13.

(26) Vickrey, T. L., Xiao, N., and Venton, B. J. Kinetics of the Dopamine Transporter in Drosophila Larva. ACS Chem. Neurosci. American Chemical Society.

(27) Xiao, N., and Venton, B. (2012) Rapid, Sensitive Detection of Neurotransmitters at Microelectrodes Modified with Self-Assembled SWCNT Forests. Anal. Chem.

(28) Borue, X., Condron, B., and Venton, B. J. (2010) Both synthesis and reuptake are critical for replenishing the releasable serotonin pool in Drosophila. J. Neurochem. 113, 188-99.

(29) Privman, E., and Venton, B. J. (2015) Comparison of dopamine kinetics in the larval Drosophila ventral nerve cord and protocerebrum with improved optogenetic stimulation. J. Neurochem. 135, 695-704.

(30) Rees, H. R., Anderson, S. E., Privman, E., Bau, H. H., and Venton, B. J. (2015) Carbon nanopipette electrodes for dopamine detection in Drosophila. Anal. Chem. 87, 3849-55.

(31) Xiao, N., and Venton, B. J. (2015) Characterization of dopamine releasable and reserve pools in Drosophila larvae using ATP/P2X2 -mediated stimulation. J. Neurochem. 134, 445-54.

(32) Koslow, S. H. (1974) Application of mass fragmentography to the quantitation of endogenous catecholamines. Biochem. Pharmacol. 23, 901-906.

(33) Arnold, E. L., and Ford, R. (1973) Determination of catechol-containing compounds in tissue samples by gas-liquid chromatography. Anal. Chem. 45, 85-89.

(34) Bergquist, J., Ściubisz, A., Kaczor, A., and Silberring, J. (2002) Catecholamines and methods for their identification and quantitation in biological tissues and fluids. $J$. Neurosci. Methods 113, 1-13. 
(35) Zapata, A., Chefer, V. I., Shippenberg, T. S., and Denoroy, L. (2009) Detection and quantification of neurotransmitters in dialysates. Curr. Protoc. Neurosci. Chapter 7, Unit 7.4.1-30.

(36) Zhao, X.-E., and Suo, Y.-R. (2008) Simultaneous determination of monoamine and amino acid neurotransmitters in rat endbrain tissues by pre-column derivatization with high-performance liquid chromatographic fluorescence detection and mass spectrometric identification. Talanta 76, 690-7.

(37) Gosetti, F., Mazzucco, E., Gennaro, M. C., and Marengo, E. (2013) Simultaneous determination of sixteen underivatized biogenic amines in human urine by HPLCMS/MS. Anal. Bioanal. Chem. 405, 907-16.

(38) Song, Y., Quan, Z., Evans, J. L., Byrd, E. A., and Liu, Y.-M. (2004) Enhancing capillary liquid chromatography/tandem mass spectrometry of biogenic amines by precolumn derivatization with 7-fluoro-4-nitrobenzoxadiazole. Rapid Commun. Mass Spectrom. 18, 989-94.

(39) Kim, J. H., Lee, J.-Y., Jin, J.-H., Park, C. W., Lee, C. J., and Min, N. K. (2012) A fully microfabricated carbon nanotube three-electrode system on glass substrate for miniaturized electrochemical biosensors. Biomed. Microdevices 14, 613-24.

(40) Sasa, S., and Blank, C. L. (1977) Determination of serotonin and dopamine in mouse brain tissue by high performance liquid chromatography with electrochemical detection. Anal. Chem. 49, 354-9.

(41) Hardie, S. L., and Hirsh, J. An improved method for the separation and detection of biogenic amines in adult Drosophila brain extracts by high performance liquid chromatography. J. Neurosci. Methods.

(42) Kennedy, R. T., and Jorgenson, J. W. (1989) Quantitative analysis of individual neurons by open tubular liquid chromatography with voltammetric detection. Anal. Chem. 61, 436-441.

(43) Cheng, F. C., Shih, Y., Liang, Y. J., Yang, L. L., and Yang, C. S. (1996) New dual electrochemical detector for microbore liquid chromatography. Determination of dopamine and serotonin in rat striatum dialysates. J. Chromatogr. B. Biomed. Appl. 682, 195-200.

(44) Cheng, F.-C., Lin, N.-N., Kuo, J.-S., Cheng, L.-J., Chang, F.-M., and Chia, L.-G. (1994) Simultaneous measurement of plasma serotonin, catecholamines, and their metabolites by anin vitro microdialysis-microbore hplc and a dual amperometric detector. Electroanalysis 6, 871-877.

(45) Ishikawa, K., and McGaugh, J. L. (1982) Simultaneous determination of monoamine transmitters, precursors and metabolites in a single mouse brain. J. Chromatogr. 229, 35-46.

(46) Donzanti, B. A., and Yamamoto, B. K. (1988) An improved and rapid HPLC-EC method for the isocratic separation of amino acid neurotransmitters from brain tissue and microdialysis perfusates. Life Sci. 43, 913-922.

(47) Seegal, R. F., Brosch, K. O., and Bush, B. (1986) High-performance liquid chromatography of biogenic amines and metabolites in brain, cerebrospinal fluid, urine and plasma. J. Chromatogr. 377, 131-44.

(48) Honegger, C. G., Burri, R., Langemann, H., and Kempf, A. (1984) Determination of neurotransmitter systems in human cerebrospinal fluid and rat nervous tissue by highperformance liquid chromatography with on-line data evaluation. J. Chromatogr. $B$ 
Biomed. Sci. Appl. 309, 53-61.

(49) Mark Wightman, R., Plotsky, P. M., Strope, E., Delcore, R., and Adams, R. N.

(1977) Liquid chromatographic monitoring of CSF metabolites. Brain Res. 131, 345-349.

(50) Church, W. H., Justice, J. B., and Neill, D. B. (1987) Detecting behaviorally relevant changes in extracellular dopamine with microdialysis. Brain Res. 412, 397-9.

(51) Chaurasia, C. S., Chen, C.-E., and Ashby, C. R. (1999) In vivo on-line HPLCmicrodialysis: simultaneous detection of monoamines and their metabolites in awake freely-moving rats. J. Pharm. Biomed. Anal. 19, 413-422.

(52) Wages, S. A., Church, W. H., and Justice, J. B. (1986) Sampling considerations for on-line microbore liquid chromatography of brain dialysate. Anal. Chem. 58, 1649-56.

(53) Parrot, S., Neuzeret, P.-C., and Denoroy, L. (2011) A rapid and sensitive method for the analysis of brain monoamine neurotransmitters using ultra-fast liquid chromatography coupled to electrochemical detection. J. Chromatogr. B. Analyt. Technol. Biomed. Life Sci. 879, 3871-8.

(54) Reinhoud, N. J., Brouwer, H.-J., van Heerwaarden, L. M., and Korte-Bouws, G. A. $\mathrm{H}$. (2013) Analysis of glutamate, GABA, noradrenaline, dopamine, serotonin, and metabolites using microbore UHPLC with electrochemical detection. ACS Chem. Neurosci. 4, 888-94.

(55) Hjertén, S. (1967) Free zone electrophoresis. Chromatogr. Rev. 9, 122-219.

(56) Jorgenson, J. W., and Lukacs, K. D. (1981) Zone electrophoresis in open-tubular glass capillaries. Anal. Chem. 53, 1298-1302.

(57) VanOrman, B. B., Liversidge, G. G., Mclntire, G. L., Olefirowicz, T. M., and Ewing, A. G. (1990) Effects of buffer composition on electroosmotic flow in capillary electrophoresis. J. Microcolumn Sep. 2, 176-180.

(58) Weinberger, R. (2000) Practical Capillary Electrophoresis. Elsevier Science.

(59) Landers, J. P. (2007) Handbook of Capillary and Microchip Electrophoresis and Associated Microtechniques, Third Edition. CRC Press.

(60) Plenert, M. L., and Shear, J. B. (2003) Microsecond electrophoresis. Proc. Natl. Acad. Sci. U. S. A. 100, 3853-7.

(61) Monnig, C. A., and Jorgenson, J. W. (1991) On-column sample gating for highspeed capillary zone electrophoresis. Anal. Chem. 63, 802-807.

(62) Tanaka, S., Kaneta, T., Taga, M., Yoshida, H., and Ohtaka, H. (1991) Capillary tube isotachophoretic separation of catecholamines using cyclodextrin in the leading electrolyte. J. Chromatogr. A 587, 364-367.

(63) Ewing, A. G., Wallingford, R. A., and Olefirowicz, T. M. (1989) Capillary electrophoresis. Anal. Chem. 61, 292A-303A.

(64) Wallingford, R. A., and Ewing, A. G. (1988) Capillary zone electrophoresis with electrochemical detection in 12.7 .mu.m diameter columns. Anal. Chem. 60, 1972-1975.

(65) Wallingford, R. A., and Ewing, A. G. (1989) Separation of serotonin from catechols by capillary zone electrophoresis with electrochemical detection. Anal. Chem. 61, 98100.

(66) Rocher, C., Bert, L., Robert, F., Trouvin, J.-H., Renaud, B., Jacquot, C., and Gardier, A. M. (1996) Microdialysis monitoring of variations in extracellular levels of serotonin, GABA and excitatory amino acids in the frontal cortex of awake rats in 
response to a single peripheral or central administration of dexfenfluramine. Brain Res. 737, 221-230.

(67) Zhou, S. Y., Zuo, H., Stobaugh, J. F., Lunte, C. E., and Lunte, S. M. (1995) Continuous in Vivo Monitoring of Amino Acid Neurotransmitters by Microdialysis Sampling with Online Derivatization and Capillary Electrophoresis Separation. Anal. Chem. 67, 594-599.

(68) Jäverfalk-Hoyes, E. M., Bondesson, U., Westerlund, D., and Andrén, P. E. (1999) Simultaneous analysis of endogenous neurotransmitters and neuropeptides in brain tissue using capillary electrophoresis - microelectrospray-tandem mass spectrometry. Electrophoresis 20, 1527-1532.

(69) Weber, P. L., O'Shea, T. J., and Lunte, S. M. (1994) Separation and quantitation of the amino acid neurotransmitters in rat brain by capillary electrophoresis. J. Pharm. Biomed. Anal. 12, 319-324.

(70) Zhang, C. X., and Thormann, W. Head-Column Field-Amplified Sample Stacking in Binary System Capillary Electrophoresis: A Robust Approach Providing over 1000-Fold Sensitivity Enhancement. Anal. Chem. American Chemical Society.

(71) Shihabi, Z. K. (2002) Transient pseudo-isotachophoresis for sample concentration in capillary electrophoresis. Electrophoresis 23, 1612-7.

(72) Mohan, D., and Lee, C. S. (2002) On-line coupling of capillary isoelectric focusing with transient isotachophoresis-zone electrophoresis: a two-dimensional separation system for proteomics. Electrophoresis 23, 3160-7.

(73) de Mello, A. J., and Beard, N. (2003) Focus. Dealing with ?real? samples: sample pre-treatment in microfluidic systems. Lab Chip 3, 11N.

(74) Kubán, P., and Hauser, P. C. (2009) Fundamentals of electrochemical detection techniques for CE and MCE. Electrophoresis 30, 3305-14.

(75) Wallenborg, S. R., Nyholm, L., and Lunte, C. E. (1999) End-column amperometric detection in capillary electrophoresis: influence of separation-related parameters on the observed half-wave potential for dopamine and catechol. Anal. Chem. 71, 544-9.

(76) Vandaveer, W. R., Pasas-Farmer, S. A., Fischer, D. J., Frankenfeld, C. N., and Lunte, S. M. (2004) Recent developments in electrochemical detection for microchip capillary electrophoresis. Electrophoresis 25, 3528-49.

(77) Voegel, P. D., and Baldwin, R. P. Electrochemical detection in capillary electrophoresis. Electrophoresis. Wiley Subscription Services, Inc., A Wiley Company.

(78) Matysik, F.-M. (2000) End-Column Electrochemical Detection for Capillary Electrophoresis. Electroanalysis 12, 1349-1355.

(79) Voegel, P. D., and Baldwin, R. P. (1997) Electrochemical detection in capillary electrophoresis. Electrophoresis 18, 2267-78.

(80) Qian, J., Wu, Y., Yang, H., and Michael, A. C. (1999) An Integrated Decoupler for Capillary Electrophoresis with Electrochemical Detection: Application to Analysis of Brain Microdialysate. Anal. Chem. 71, 4486-4492.

(81) Sloss, S., and Ewing, A. G. (1993) Improved method for end-column amperometric detection for capillary electrophoresis. Anal. Chem. 65, 577-581.

(82) Osboum, D. M., and Lunte, C. E. (2001) Cellulose acetate decoupler for on-column electrochemical detection in capillary electrophoresis. Anal. Chem. 73, 5961-4. 
(83) Park, S., and Lunte, C. E. (1995) A perfluorosulfonated ionomer end-column electrical decoupler for capillary electrophoresis/electrochemical detection. Anal. Chem. 67, 4366-4370.

(84) Huang, X., Zare, R. N., Sloss, S., and Ewing, A. G. (1991) End-column detection for capillary zone electrophoresis. Anal. Chem. 63, 189-192.

(85) Dolník, V., Liu, S., and Jovanovich, S. (2000) Capillary electrophoresis on microchip. Electrophoresis 21, 41-54.

(86) Lacher, N. A., Lunte, S. M., and Martin, R. S. (2004) Development of a microfabricated palladium decoupler/electrochemical detector for microchip capillary electrophoresis using a hybrid glass/poly(dimethylsiloxane) device. Anal. Chem. 76, 2482-91.

(87) Martin, R. S., Gawron, A. J., Lunte, S. M., and Henry, C. S. (2000) Dual-Electrode Electrochemical Detection for Poly(dimethylsiloxane)-Fabricated Capillary Electrophoresis Microchips. Anal. Chem. 72, 3196-3202.

(88) Wang, J., Polsky, R., Tian, B., and Chatrathi, M. P. (2000) Voltammetry on Microfluidic Chip Platforms. Anal. Chem. 72, 5285-5289.

(89) Henry, C. S., Zhong, M., Lunte, S. M., Kim, M., Bau, H., and Santiago, J. J. (1999) Ceramic microchips for capillary electrophoresis-electrochemistry. Anal. Commun. 36, 305-307.

(90) Luo, Y., Wu, D., Zeng, S., Gai, H., Long, Z., Shen, Z., Dai, Z., Qin, J., and Lin, B. (2006) Double-cross hydrostatic pressure sample injection for chip CE: variable sample plug volume and minimum number of electrodes. Anal. Chem. 78, 6074-80.

(91) Backofen, U., Matysik, F.-M., and Lunte, C. E. (2002) A Chip-Based Electrophoresis System with Electrochemical Detection and Hydrodynamic Injection. Anal. Chem. 74, 4054-4059.

(92) Matysik, F.-M. (2007) Advances in amperometric and conductometric detection in capillary and chip-based electrophoresis. Microchim. Acta 160, 1-14.

(93) Wang, M., Hershey, N. D., Mabrouk, O. S., and Kennedy, R. T. (2011) Collection, storage, and electrophoretic analysis of nanoliter microdialysis samples collected from awake animals in vivo. Anal. Bioanal. Chem. 400, 2013-2023.

(94) Kuklinski, N. J., Berglund, E. C., Engelbreksson, J., and Ewing, A. G. Determination of salsolinol, norsalsolinol, and twenty-one biogenic amines using micellar electrokinetic capillary chromatography-electrochemical detection. Electrophoresis. WILEY-VCH Verlag.

(95) Kuklinski, N. J., Berglund, E. C., Engelbrektsson, J., and Ewing, A. G. Biogenic Amines in Microdissected Brain Regions of Drosophila melanogaster Measured with Micellar Electrokinetic Capillary Chromatography-Electrochemical Detection. Anal. Chem. American Chemical Society.

(96) Fischer, D. J., Vandaveer, W. R., Grigsby, R. J., and Lunte, S. M. (2005) Pyrolyzed Photoresist Carbon Electrodes for Microchip Electrophoresis with Dual-Electrode Amperometric Detection. Electroanalysis 17, 1153-1159.

(97) Wallingford, R. A., and Ewing, A. G. (1988) Amperometric detection of catechols in capillary zone electrophoresis with normal and micellar solutions. Anal. Chem. 60, 258263.

(98) Chien, J. B., Wallingford, R. A., and Ewing, A. G. (1990) Estimation of Free 
Dopamine in the Cytoplasm of the Giant Dopamine Cell of Planorbis corneus by Voltammetry and Capillary Electrophoresis. J. Neurochem. 54, 633-638.

(99) Berglund, E. C., Kuklinski, N. J., Karagündüz, E., Ucar, K., Hanrieder, J. ., and Ewing, A. G. Freeze-Drying as Sample Preparation for Micellar Electrokinetic Capillary Chromatography-Electrochemical Separations of Neurochemicals in Drosophila Brains. Anal. Chem. American Chemical Society.

(100) Saylor, R. A., Reid, E. A., and Lunte, S. M. (2015) Microchip electrophoresis with electrochemical detection for the determination of analytes in the dopamine metabolic pathway. Electrophoresis 36, 1912-9.

(101) Fang, H., Vickrey, T. L., and Venton, B. J. (2011) Analysis of Biogenic Amines in a Single Drosophila Larva Brain by Capillary Electrophoresis with Fast-Scan Cyclic Voltammetry Detection. Anal. Chem. 83, 2258-2264.

(102) Neckameyer, W. S., Woodrome, S., Holt, B., and Mayer, A. Dopamine and senescence in Drosophila melanogaster. Neurobiol. Aging.

(103) Borycz, J., Borycz, J. A., Kubów, A., Lloyd, V., and Meinertzhagen, I. A. (2008) Drosophila $A B C$ transporter mutants white, brown and scarlet have altered contents and distribution of biogenic amines in the brain. J. Exp. Biol. 211, 3454-66.

(104) Hashemi, P., Dankoski, E. C., Wood, K. M., Ambrose, R. E., and Wightman, R. M. (2011) In vivo electrochemical evidence for simultaneous 5-HT and histamine release in the rat substantia nigra pars reticulata following medial forebrain bundle stimulation. $J$. Neurochem. 118, 749-59.

(105) Samaranayake, S., Abdalla, A., Robke, R., Wood, K. M., Zeqja, A., and Hashemi, $P$. (2015) In vivo histamine voltammetry in the mouse premammillary nucleus. Analyst $140,3759-65$.

(106) Jones, L. J., McCutcheon, J. E., Young, A. M. J., and Norton, W. H. J. (2015) Neurochemical measurements in the zebrafish brain. Front. Behav. Neurosci. 9, 246.

(107) Stuart, A. E. (1999) From Fruit Flies to Barnacles, Histamine Is the Neurotransmitter of Arthropod Photoreceptors. Neuron 22, 431-433.

(108) Benarroch, E. E. (2010) Histamine in the CNS: Multiple functions and potential neurologic implications. Neurology 75, 1472-1479.

(109) Borycz, J., Vohra, M., Tokarczyk, G., and Meinertzhagen, I. A. (2000) The determination of histamine in the Drosophila head. J. Neurosci. Methods 101, 141-148.

(110) Borycz, J., Borycz, J. A., Loubani, M., and Meinertzhagen, I. A. (2002) tan and ebony Genes Regulate a Novel Pathway for Transmitter Metabolism at Fly Photoreceptor Terminals. J. Neurosci. 22, 10549-10557.

(111) Gavin, B. A., Arruda, S. E., and Dolph, P. J. (2007) The role of carcinine in signaling at the Drosophila photoreceptor synapse. PLoS Genet. 3, e206.

(112) Takahashi, A. (2013) Pigmentation and behavior: potential association through pleiotropic genes in Drosophila. Genes Genet. Syst. 88, 165-74.

(113) Monastirioti, M. (1999) Biogenic amine systems in the fruit fly Drosophila melanogaster. Microsc. Res. Tech. 45, 106-21.

(114) Aust, S., Brüsselbach, F., Pütz, S., and Hovemann, B. T. (2010) Alternative Tasks of Drosophila Tan in Neurotransmitter Recycling Versus Cuticle Sclerotization Disclosed by Kinetic Properties. J. Biol. Chem. 285, 20740-20747. 
(115) Wittkopp, P. J., Williams, B. L., Selegue, J. E., and Carroll, S. B. (2003) Drosophila pigmentation evolution: divergent genotypes underlying convergent phenotypes. Proc.

Natl. Acad. Sci. U. S. A. 100, 1808-13.

(116) Brody, T. (1999) The Interactive Fly: gene networks, development and the Internet. Trends Genet. 15, 333-4.

(117) Melzig, J., Burg, M., Gruhn, M., Pak, W. L., and Buchner, E. (1998) Selective Histamine Uptake Rescues Photo- and Mechanoreceptor Function of Histidine Decarboxylase-Deficient Drosophila Mutant. J. Neurosci. 18, 7160-7166.

(118) Chaturvedi, R., Reddig, K., and Li, H.-S. (2014) Long-distance mechanism of neurotransmitter recycling mediated by glial network facilitates visual function in Drosophila. Proc. Natl. Acad. Sci. 111, 2812-2817.

(119) Pérez, M., Schachter, J., and Quesada-Allué, L. A. (2004) Constitutive activity of $\mathrm{N}$-beta-alanyl-catecholamine ligase in insect brain. Neurosci. Lett. 368, 186-91.

(120) Ziegler, A. B., Brüsselbach, F., and Hovemann, B. T. (2013) Activity and coexpression of Drosophila black with ebony in fly optic lobes reveals putative cooperative tasks in vision that evade electroretinographic detection. J. Comp. Neurol. $521,1207-24$.

(121) True, J. R., Yeh, S.-D., Hovemann, B. T., Kemme, T., Meinertzhagen, I. A., Edwards, T. N., Liou, S.-R., Han, Q., and Li, J. (2005) Drosophila tan encodes a novel hydrolase required in pigmentation and vision. PLoS Genet. 1, e63.

(122) Chang, S.-Y., Jay, T., Muñoz, J., Kim, I., and Lee, K. H. (2012) Wireless fast-scan cyclic voltammetry measurement of histamine using WINCS--a proof-of-principle study. Analyst 137, 2158-65.

(123) Pihel, K., Hsieh, S., Jorgenson, J. W., and Wightman, R. M. (1995)

Electrochemical detection of histamine and 5-hydroxytryptamine at isolated mast cells. Anal. Chem. 67, 4514-4521.

(124) Bullock, J. A., and Yuan, L.-C. (1991) Free solution capillary electrophoresis of basic proteins in uncoated fused silica capillary tubing. J. Microcolumn Sep. 3, 241-248.

(125) Dos Santos, G., Schroeder, A. J., Goodman, J. L., Strelets, V. B., Crosby, M. A., Thurmond, J., Emmert, D. B., and Gelbart, W. M. (2015) FlyBase: introduction of the Drosophila melanogaster Release 6 reference genome assembly and large-scale migration of genome annotations. Nucleic Acids Res. 43, D690-7.

(126) Wright, T. R. (1987) The genetics of biogenic amine metabolism, sclerotization, and melanization in Drosophila melanogaster. Adv. Genet. 24, 127-222.

(127) Weigmann, K., Klapper, R., Strasser, T., Rickert, C., Technau, G., Jäckle, H., Janning, W., and Klämbt, C. (2003) FlyMove--a new way to look at development of Drosophila. Trends Genet. 19, 310-1.

(128) Lanet, E., and Maurange, C. (2014) Building a brain under nutritional restriction: insights on sparing and plasticity from Drosophila studies. Front. Physiol. 5, 117. 
Chapter 2. Analysis of Neurotransmitter Tissue Content of Drosophila melanogaster in different life stages 


\subsection{Abstract}

Drosophila melanogaster is a widely used model organism for studying neurological diseases with similar neurotransmission to mammals. While both larva and adult Drosophila have central nervous systems, not much is known about how neurotransmitter tissue content changes through development. In this chapter, we quantified tyramine, serotonin, octopamine, and dopamine in larval, pupal, and adult fly brains using capillary electrophoresis coupled to fast-scan cyclic voltammetry. Tyramine and octopamine content varied between life stages, with almost no octopamine being present in the pupa, while tyramine levels in the pupa were very high. Adult females had significantly higher dopamine content than males, but no other neurotransmitters were dependent on sex in the adult. Understanding the tissue content of different life stages will be beneficial for future work comparing the effects of diseases on tissue content throughout development. This was published in ACS Chem. Neurosci., 2015, 6 (1), pp $117-123 .^{1}$ 


\subsection{Introduction}

Drosophila melanogaster has four developmental stages: embryo, larva, pupa, and adult. The larval stage is divided into three instars, during which larva feed and move. ${ }^{2}$ During pupation, Drosophila attaches to a hard surface and becomes immobile, and larval structures are replaced by adult structures. The pupa then ecloses and emerges as an adult. The central nervous system of Drosophila changes during development. In late embryonic development, the primary neurons of the larval brain are developed and the basic structure of the adult brain is laid out. ${ }^{3,4}$ As the larvae grow, neurons that will persist into the adulthood develop and embryonic stage neurons are pruned. ${ }^{5}$ During pupation, larval neuroblasts become adult neurons and there is proliferation and growth of neurons in the brain. ${ }^{6}$ Similar to mammals, Drosophila have dopamine and serotonin neurotransmission, which is regulated by transporters that are homologous to human transporters. ${ }^{7,8}$ Drosophila also have tyramine and octopamine, present in humans only as trace amines, that have homologous functions to epinephrine and norepinephrine in mammals. ${ }^{9}$ Despite much research into Drosophila development, changes in neurotransmitter tissue content over its life cycle have not been characterized.

Neurotransmission has been studied in Drosophila by measuring stimulated release of dopamine or serotonin, but tissue content is required to understand how neurotransmitter release relates to the releasable pool of neurotransmitter. ${ }^{10,11}$ Separation techniques such as high-performance liquid chromatography (HPLC) and capillary electrophoresis (CE) have been used for tissue content analysis. HPLC requires pooled samples (3-4 heads) because of high mass detection limits. ${ }^{12,13}$ With CE, a single head or brain can be analyzed. ${ }^{14,15}$ Studying brains is preferred due to the presence of neurotransmitters in the cuticle and the eyes of Drosophila. Previous capillary electropohresis studies used amperometric electrochemical detection, which is 
sensitive but provides little chemical information about the analyte observed. Our lab has coupled CE to fast-scan cyclic voltammetry detection (CE-FSCV), which produces characteristic cyclic voltammograms that aid in analyte identification. ${ }^{16,17}$ Previous studies focused on detection of serotonin, octopamine, and dopamine in larval Drosophila but no comparisons have been made to other life stages. ${ }^{17}$

The goal of this study was to compare neurotransmitter tissue content in larval, pupal, and adult wild type (Canton S) brains using CE-FSCV. Tyramine, serotonin, octopamine, and dopamine were quantified and the limits of detection in the 5-16 pg range were sufficient for analysis of single brains. Tyramine and octopamine varied significantly throughout the development of Drosophila; in the pupa, tyramine content was high while octopamine content was undetectable. The amount of serotonin or dopamine did not significantly change at any life stage. Adult females had significantly more dopamine than males. Thus, neurotransmitter content can vary by life stage and sex.

\subsection{Results and Discussion}

\subsubsection{Capillary electrophoresis and fast scan cyclic voltammetry}

Capillary electrophoresis with fast scan voltammetry (CE-FSCV) separates

analytes quickly and allows small samples to be detected with identification provided by the cyclic voltammograms. The electrode was scanned from $-0.4 \mathrm{~V}$ to $1.4 \mathrm{~V}$ and back at $400 \mathrm{~V} / \mathrm{s}$ every $100 \mathrm{~ms}$ for high sensitivity detection of tyramine and octopamine. In order to maximize sensitivity, field amplified sample injection was used by having a high conductivity separation buffer and a low conductivity sample matrix. ${ }^{18,19}$ Analytes in this low conductivity solution experience high electric fields and migrate rapidly towards the inlet of the capillary which pre-concentrates analytes and decreases peak widths. For 
the sample matrix, we used deionized water with perchloric acid, which prevents degradation of monoamine neurotransmitters. ${ }^{20}$

\subsubsection{Separations of tyramine, serotonin, octopamine and dopamine}

Figure 1 shows the

separation and detection of

$100 \mathrm{nM}$ of tyramine,

serotonin, octopamine, and

dopamine in $10 \mu \mathrm{L} 0.5 \mathrm{mM}$

perchloric acid, which takes

under six minutes. At the top

of Figure 1, the

characteristic cyclic

voltammogram for each

analyte is shown in

migration order: tyramine

(TA), serotonin (5-HT),

octopamine (OA), and

dopamine (DA). Tyramine

and octopamine each have

two peaks, with the primary

oxidation peak at $1.3 \mathrm{~V}$ and

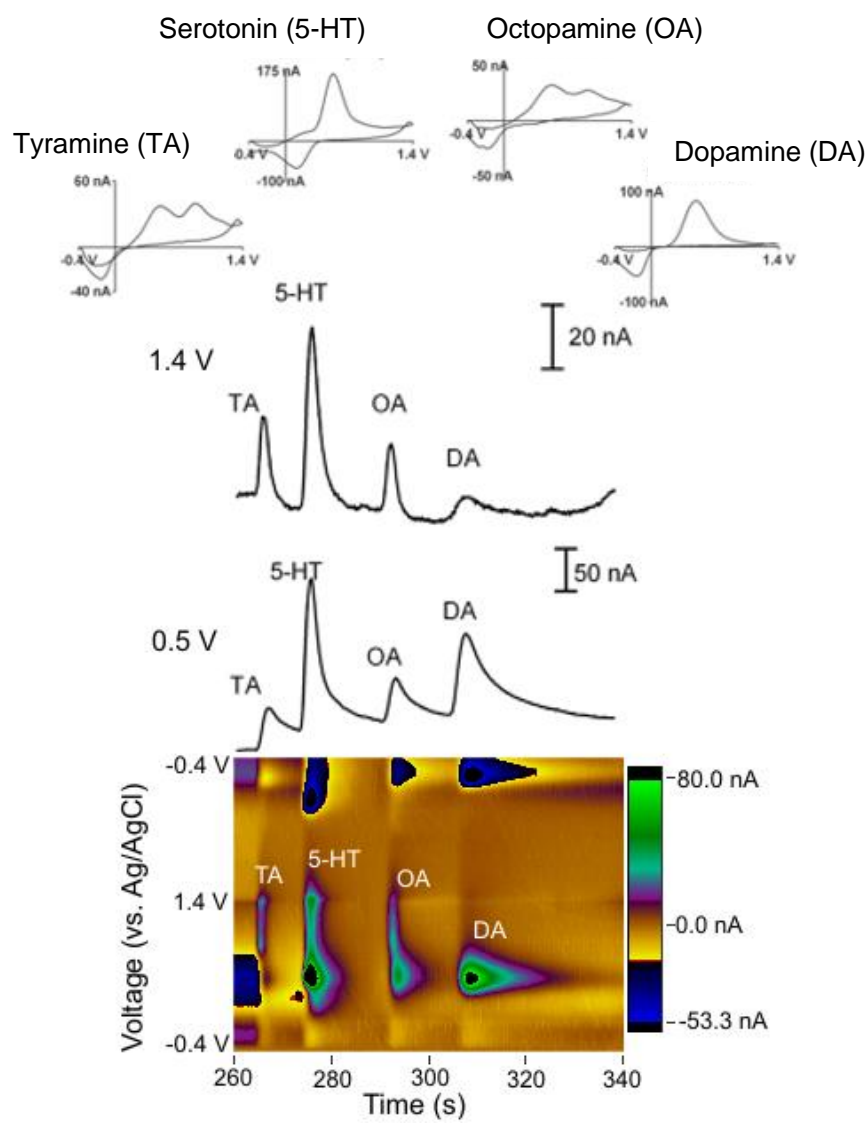

Figure 2-1: Cyclic voltammograms (CVs), electropherograms, and false color plots for separation of a $100 \mathrm{nM}$ standard mix.

Electropherograms at two different potentials are shown so that all oxidation potential peaks can be seen. Neurotransmitters are labeled on the color plot: tyramine (TA), serotonin (5-HT), octopamine (OA), and dopamine (DA). Adapted with permission from Denno et. al 2015.

a secondary peak around $0.5 \mathrm{~V}$. Both are well separated which avoids confusion of analyte identity. Serotonin has a single, sharp peak at $0.5 \mathrm{~V}$. Dopamine has a wider oxidation peak around $0.6 \mathrm{~V}$ and a reduction peak at $-0.2 \mathrm{~V}$. Below the cyclic voltammograms are two electropherogram traces at different voltages: $1.4 \mathrm{~V}$ and $0.5 \mathrm{~V}$. 
At $1.4 \mathrm{~V}$, tyramine, serotonin, and octopamine are easily visible and at $0.5 \mathrm{~V}$ all analytes are visible. The peaks for tyramine and octopamine at $0.5 \mathrm{~V}$ are secondary peaks and thus they last longer at the electrode. For quantitation, primary peaks from the cyclic voltammograms are used.

The current vs. time electropherogram (Figure 1) traces differ from those obtained using amperometry, as with amperometry all analytes oxidizing at or below the applied potential would be present. For cyclic voltammetry, only compounds with a current at that given potential are present; thus not every compound is seen on every trace. In order to view all potentials at the same time, a false color plot is used (Figure 1). The color plot at the bottom of Figure 1 plots applied potential on the y-axis, time on the $x$ axis, and current in false color. The green areas show positive current (oxidation) and the blue areas show negative current (reduction). In the color plots from real samples (Figures $2 \mathrm{~A}-\mathrm{C}$ ), other areas of color appear due to unknown compounds present in the fly. The color plot helps identify the analytes of interest and discriminate them from interferents.

Using $100 \mathrm{nM}$ standards, limits of detection were calculated for the analytes. For tissue content studies, the results are often reported in absolute amount of neurotransmitter and the LODs in pg were $16 \pm 2$ for tyramine, $5.3 \pm 0.9$ for serotonin, 12 \pm 3 for octopamine, and $5 \pm 2$ for dopamine $(n=3)$. These limits of detection are comparable to previously published work using our CE-FSCV system. ${ }^{17}$

\subsubsection{Separations of neurotransmitters in different life stages of Drosophila}


Figure $2 A-C$ show separations of neurotransmitters in $3^{\text {rd }}$ instar wandering larva,

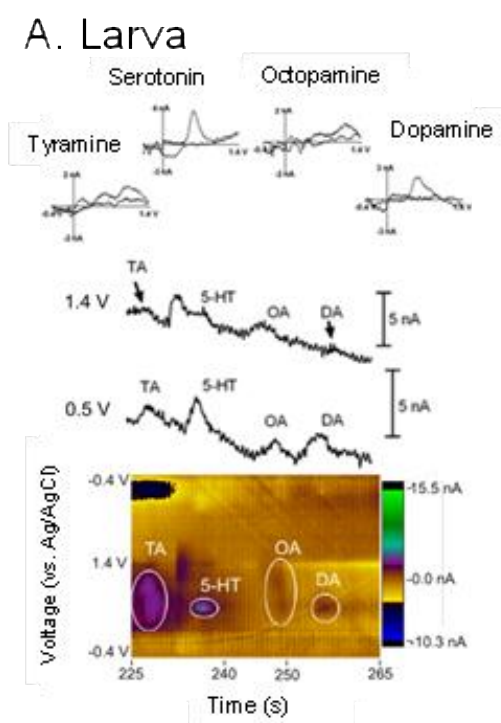

B. Pupa

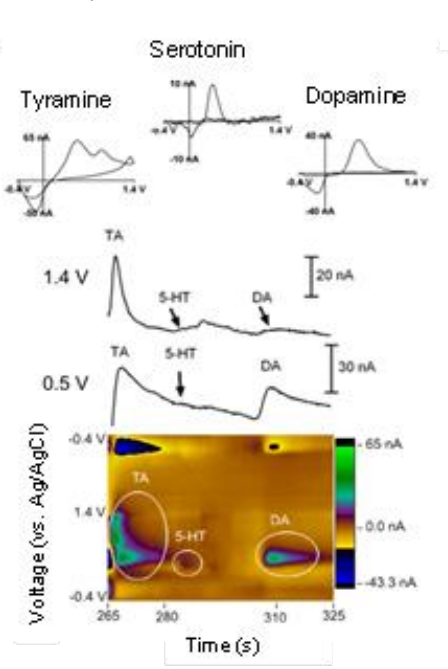

C. Adult

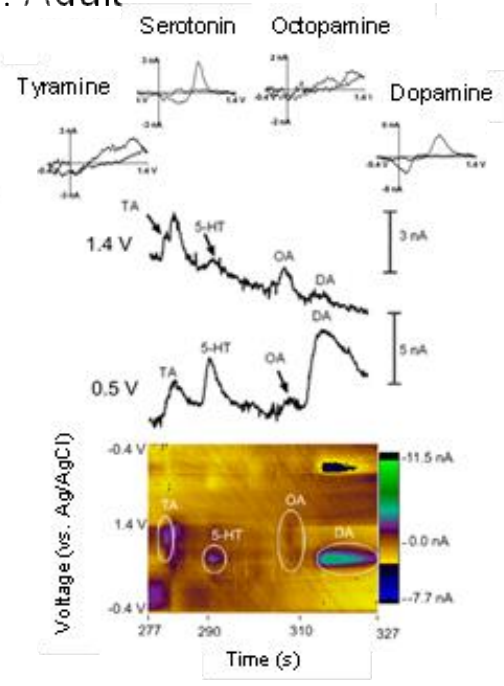

Figure 2-2: Cyclic voltammograms (CVs), electropherograms, and false color plots for separations of various tissues. A. 3rd instar larva central nervous system B. Late stage pupa brain and C. $72 \mathrm{hr}$ adult male brain. Neurotransmitters are labeled on the color plot: tyramine (TA), serotonin (5-HT), octopamine (OA), and dopamine (DA). Adapted with permission from Denno et. al 2015.

late pupa, and 72 hour old adult Canton S. In the larva (Figure 2A), tyramine, serotonin, octopamine, and dopamine were all present in low amounts. The amount of neurotransmitter in Figure 2A was 18.2 pg tyramine, 12.9 pg serotonin, $16.7 \mathrm{pg}$ octopamine, and $16.7 \mathrm{pg}$ dopamine. In the pupa (Figure 2B), only three peaks were present: tyramine, serotonin, and dopamine. There were no peaks for octopamine where it should migrate. The concentrations were higher in the pupa than the larva, especially for tyramine (451 pg) and dopamine (103 pg). In the adult (Figure 2C), all four neurotransmitters were present. The amount of neurotransmitter in this adult was 56.8 pg tyramine, $22.5 \mathrm{pg}$ serotonin, $41.0 \mathrm{pg}$ octopamine, and $69.2 \mathrm{pg}$ dopamine. While migration times slightly varied due to different capillaries or differences in EOF, migration order is the same for all separations. 
Table 2-1: Average tissue content values in larva, pupa and adult.

\begin{tabular}{lcrrr} 
Life Stage & \multicolumn{1}{l}{$\begin{array}{l}\text { Tyramine } \\
\text { (TA) }\end{array}$} & \multicolumn{1}{l}{$\begin{array}{l}\text { Serotonin (5- } \\
\text { HT) }\end{array}$} & $\begin{array}{l}\text { Octopamine } \\
\text { (OA) }\end{array}$ & \multicolumn{1}{l}{$\begin{array}{l}\text { Dopamine } \\
\text { (DA) }\end{array}$} \\
Larva $(\mathbf{n}=8)$ & $66 \pm 18 \mathrm{pg}$ & $18 \pm 3 \mathrm{pg}$ & $58 \pm 19 \mathrm{pg}$ & $41 \pm 15 \mathrm{pg}$ \\
Pupa $(\mathbf{n}=7-8)$ & $272 \pm 57 \mathrm{pg}$ & $21 \pm 3 \mathrm{pg}$ & Not detected & $137 \pm 57 \mathrm{pg}$ \\
Adult $(\mathbf{n}=10)$ & $67 \pm 8 \mathrm{pg}$ & $22 \pm 5 \mathrm{pg}$ & $45 \pm 7 \mathrm{pg}$ & $85 \pm 13 \mathrm{pg}$
\end{tabular}

Errors are standard error of the mean (SEM). Values were tested for outliers using ROUT $(\mathrm{Q}=0.1 \%)$, and definitive outliers were removed.

Our tissue content values (Table 1) are similar to those previously reported. However, no previous reports of any pupal values are available. Tissue content of dopamine in larva (41 pg/brain) was higher than previously reported (19 $\pm 2 \mathrm{pg} / \mathrm{brain}) .{ }^{17}$ In adult brains, tissue content (85 pg/brain) agreed with some previously published values $(80.7 \mathrm{pg} / \mathrm{brain})^{21}$ but not others $(10 \pm 2 \mathrm{pg} / \mathrm{brain}) .{ }^{22}$ Differences in values here may be due to sample preparation or the inclusion of small parts of the cuticle in our samples. Whole head content was much higher than our value $(398 \pm 137 \mathrm{fmol} / \mathrm{brain})^{15}$ which is due to the inclusion of cuticle in the sample. ${ }^{23}$

Serotonin tissue content values for larvae (18 pg/brain) were similar to our previous work (23 $\pm 2 \mathrm{pg} / \mathrm{brain})^{17}$ and those previously reported elsewhere (13 pg/brain). ${ }^{24}$ In adult brain samples, there was good agreement between our serotonin values (22 pg/brain) and previously published work (25 pg/brain).${ }^{25}$ Serotonin content in brains was lower than previously reported in whole head samples (1867 \pm 317 $\mathrm{pg} /$ head). ${ }^{15}$

Our tyramine value for adults was higher (67 pg/brain) than a previously reported value $(7.6 \mathrm{pg} / \mathrm{brain})^{21}$, but was lower than values for tyramine from whole heads $(109 \pm$ 
$69 \mathrm{pg}) .{ }^{15}$ No previous average values for tyramine have been reported in lavae.

Differences in tissue content may be due to age of flies collected. In the same paper with the low tyramine value in adults, a high value for octopamine (263 pg/brain) was reported. ${ }^{21}$ Since tyramine is the synthetic precursor to octopamine, high synthesis of octopamine could lead to smaller amounts of tyramine. Octopamine tissue content in larva (58 pg/brain) was not significantly different from our previously published work (38 $\pm 3 \mathrm{pg} / \mathrm{brain}) .{ }^{17}$ Other labs have reported higher values for adults $(263 \mathrm{pg} / \mathrm{brain})^{21}$ than our whole brain values (45 pg/brain) but Hirsh et al. ${ }^{23}$ have shown evidence of an unidentified analyte that coelutes with octopamine, causing overestimation of octopamine tissue content. By using FSCV, we were certain of our octopamine peak identification. Our octopamine values were also lower than those of whole head samples $(260 \pm 61 \mathrm{pg} / \mathrm{head})^{15}$, but this could be due to the inclusion of Type II terminals in head samples, which are octopaminergic terminals to muscles that occur outside the brain.

\subsubsection{Comparisons of neurotransmitters in different life stages of Drosophila}

Figure 3 shows the average amount of tyramine $(A)$, serotonin $(B)$, octopamine (C), and dopamine (D) across the three life stages studied. The average values from each life stage were compared using one-way ANOVA with Bonferonni post test. Dopamine $(F(2,22)=2.373, p=0.1166)$ and serotonin $(F(2,23)=0.2022, p=0.8184)$ tissue content do not significantly change over the course of development. Tyramine significantly varied by development stage $(F(2,23)=13.01, p=0.0002)$ and content was significantly higher in the pupa than either the larva $(p=0.0007)$ or the adult $(p=$ $0.0004)$. Octopamine also varied by life stage $(F(2,23)=7.173, p=0.0038)$, with the pupa having significantly less octopamine than either the larva $(p=0.0046)$ or the adult $(p=0.0232)$. 
The largest differences over development are observed in tyramine and octopamine, where pupae have large amounts of tyramine but almost no octopamine. Octopamine is responsible for locomotion, the fight or flight response, aggression, and feeding in Drosophila and other arthopods. ${ }^{9,26}$ Tyramine may inhibit locomotion and other octopamine

mediated behaviors,

especially in larvae. ${ }^{27}$

During pupation, there is

no movement or feeding;

thus, many of the

behaviors modulated by

octopamine do not occur.

In addition, high levels of

tyramine might serve to

inhibit movement in the

pupa.

Tyramine

synthesis is controlled by

tyrosine decarboxylase
A.

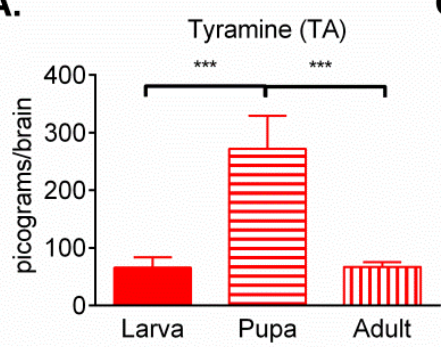

C.

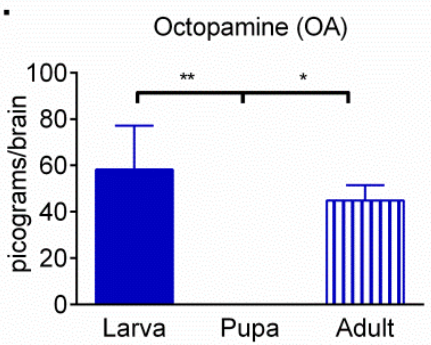

B.

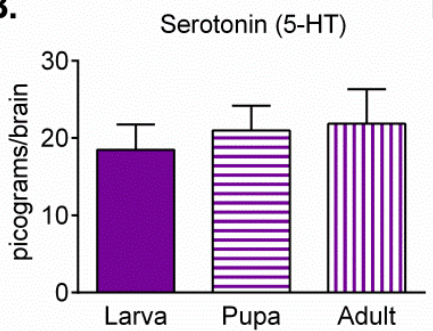

D.

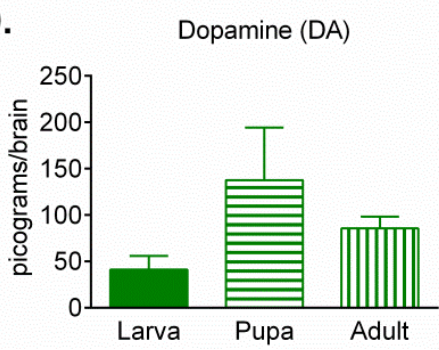

Figure 2-3: Comparisons of average tissue content across the three tested life stages, larva $(n=8)$, pupa $(n=7-8)$, adult $(n=10)$ A. Tyramine B. Serotonin C. Octopamine and D. Dopamine. One way ANOVA with Bonferroni's posttest were used to compare the tissue content of each life stage. In $A(F(2,23)=13.01, p=0.0002)$, there is significantly more tyramine in the pupa than the larva $(p=0.0007)$ and the adult $(p=0.0004)$. In $B(F(2,23)=0.2022, p=0.8184)$, no difference between anylife stages was observed. In $C(F(2,23)=$ $7.173, p=0.0038)$, both the larva $(p=0.0046)$ and the adult $(p=$ $0.0232)$ had significantly more octopamine than the pupa, $\ln D(F(2$, $22)=2.373, p=0.1166$ ), no significant difference was observed. Adapted with permission from Denno et. al 2015.

(DmellTdc2), which is expressed at low levels throughout development. ${ }^{28}$ However, the enzymatic activity of Tdc2 is not known and may be high despite low levels of expression. Tyramine is the synthetic precursor to octopamine, therefore if octopamine is not synthesized, more tyramine may be present. However, the large difference in tyramine concentration is likely not due solely to reduced synthesis of octopamine, as the amount of tyramine in pupa is greater than the combined amount of octopamine and tyramine in the larva. Octopamine is synthesized from tyramine via the enzyme 
tyramine- $\beta$-hydoxylase (DmellTbh). Larva and adults have similar gene expression for Tbh and pupa at stage 8 (P8) have moderate expression. ${ }^{28}$ However, we measured content at P10, where expression is not known and the enzyme activity might not follow the gene expression. Thus, the observed differences in tissue content are not easily predicted by changes in synthesis enzyme gene expression.

Receptor expression can also vary by developmental stage. Increases in the expression of tyramine receptors (Amtyr1, similar to DmellTyrR) in the pupal stage have been observed in Apis mellifera (the honey bee). ${ }^{29}$ The expression pattern of TyrR in Drosophila closely follow those of Apis mellifera. ${ }^{29}$ While receptor expression does not necessarily mean increased content, it may be a good indicator of increased effects of tyramine in pupae. Drosophila has several $\beta$-adrenergic receptors for octopamine $(\mathrm{Oct} \beta r)$ that have different expression patterns throughout the central nervous system. For all three of these receptors $(\operatorname{Oct} \beta 1 R, O \operatorname{ct} \beta 2 R, O \operatorname{ct} \beta 3 R)$, gene expression in the $P 10$ stage was barely detectable and was significantly higher in the adult. ${ }^{30}$ Furthermore, the expression of Oct $\beta 2 R$ was much higher than in the $3^{\text {rd }}$ instar larva than any pupal stage. ${ }^{30}$ Thus, the levels of receptor expression for Oct $\beta 2 R$ match with the tissue content studies, suggesting little octopaminergic signaling occurs in pupae.

\subsubsection{Comparisons of neurotransmitters in male and female adult Drosophila}

Figure 4 shows comparisons of tyramine, serotonin, octopamine, and dopamine tissue content in male and female adult Canton $\mathrm{S}$ flies. Only dopamine differs significantly, with more in females than males $(p=0.0383$, unpaired t-test). Higher dopamine content in females has also previously been observed in rats ${ }^{31}$ and Manduca sexta (Tobacco Hornworm) ${ }^{32}$. In Drosophila, increased dopamine in males alters courtship behavior. ${ }^{33}$ Dopamine depletion in females is linked to reduced sexual receptivity ${ }^{34}$ and dopamine is required for female flies to produce pheromones ${ }^{35}$. 
Therefore, higher dopamine levels in female Canton S flies are likely linked to reproduction.

Tissue content

provides a snapshot of

neurotransmitter

availability and relates to

synthesis and tissue

stores in the brain. In

humans and rats ${ }^{36,37}$, sex

related differences are

related to neuroanatomy,

kinetics of clearance and

re-uptake, and

metabolism of

neurotransmitters. ${ }^{38}$
A.

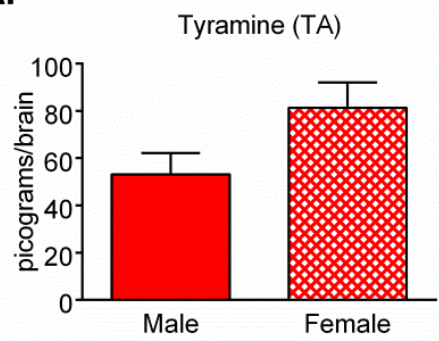

B.

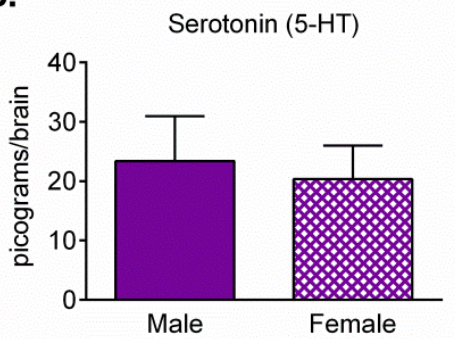

C.

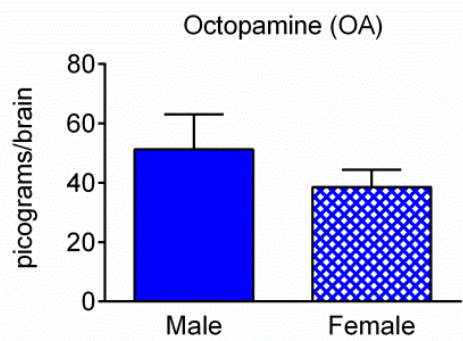

D.

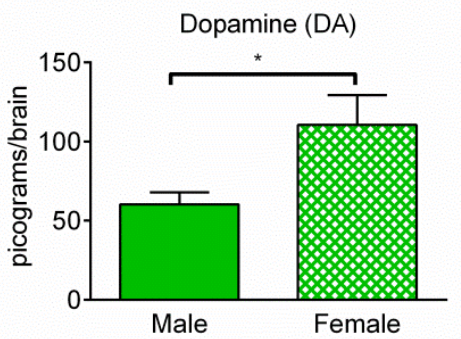

Figure 2-4: Comparisons of tissue content in male $(n=5)$ and female $(n=5)$ adult Canton $S$ brains for $A$. tyramine, B. serotonin, C. octopamine, and D. dopamine. Unpaired t-tests were used to determine if the average content of a given neurotransmitter was significantly different in male or female. For tyramine ( $P=0.0793)$, octopamine $(P=0.3671)$, and serotonin $(P=0.7566)$, there was no significant difference in tissue content between the two sexes. For dopamine, females had significantly more dopamine than males $(\mathrm{P}=$ 0.0383). Adapted with permission from Denno et. al 2015.

Future work with

metabolic and transporter Drosophila mutants to determine the impact of these mutations on neurotransmitter tissue content is important. In addition, behavioral phenotypes in mutants, such as sex-atypical courting ${ }^{33,39}$, and aggressive behaviors ${ }^{27,40-}$ ${ }^{42}$, could signify differences in neurotransmitter content. These mutants could be examined for sex differences and combined with studies of in situ monitoring of reuptake and release kinetics. Understanding the tissue content of wild-type flies can facilitate studies of age related changes in neurotransmitter content or disease-mediated changes in content, such as in Parkinson's disease. 


\subsection{Conclusions}

Tissue content analysis of single larva, pupa, and adult brains by CE-FSCV provides sensitive and accurate determination tyramine, serotonin, octopamine, and dopamine. Furthermore, identification of these neurotransmitters via characteristic cyclic voltammograms provides confidence in peak assignments. Tyramine and octopamine vary significantly over the development of Drosophila, with high levels tyramine levels but low octopamine levels in pupae. In adult Drosophila, dopamine tissue content is higher in females than males. In the future, CE-FSCV could be used to understand the long term effects of aging or disease, such as Parkinson's disease, on tissue content.

\subsection{Methods}

\subsubsection{Chemicals}

Tyramine, octopamine, serotonin, dopamine, and perchloric acid were purchased from Sigma (St. Louis, MO). $10 \mathrm{mM}$ stock solutions of neurotransmitters were prepared in $0.1 \mathrm{M}$ perchloric acid. The separation buffer was $200 \mathrm{mM} \mathrm{NaH}{ }_{2} \mathrm{PO}_{4}(\mathrm{pH}$ 3.0). The detection cell buffer was $100 \mathrm{mM} \mathrm{NaH}_{2} \mathrm{PO}_{4}(\mathrm{pH}$ 7.45). Buffer solutions for $\mathrm{CE}$ were filtered with a 0.2- $\mu$ m nylon filter (Alltech, Deerfield, IL).

\subsubsection{Fly Homogenate Preparation}

Phosphate buffered saline solution ( $\mathrm{pH} 7.4)$, with trehalose and glucose was used for dissections. Canton S fly stocks were supplied by the Drosophila Stock Center (Bloomington, IN). For larvae, the entire central nervous system (CNS) of a 5-day old, 3rd instar Drosophila larva was removed and placed in a Petri dish containing $4 \mathrm{~mL}$ dissection buffer. For pupa, the pupal case was opened. The head was then removed from the body and any cuticle and visible glial sheath was removed from the brain. For 
adults, the head was removed from the body, and trachea, cuticle, eyes, and any visible glial sheath was removed.

Sample vials were made by rotating the trimmed end of a gel-loading pipette tip (Eppendorf $20 \mu \mathrm{L}$ Microloader tips) in a flame to seal the end. A new vial was used for each sample. The CNS was transferred from the Petri dish via pipette, with minimal dissection buffer, to a sample vial containing $10 \mu \mathrm{L}$ of $0.5 \mathrm{mM}$ perchloric acid. The sample vial was centrifuged at 9,000 rpm for 2 min at room temperature. A silver wire was used as a pestle to break up the CNS. The sample vial was then sonicated in a bath sonicator (Fisher Scientific FS30 ) for 15 minutes and then inverted over a centrifugal filter (Ultrafree Centrifugal Filters, $0.22 \mu \mathrm{m}$, Millipore, Billerica, MA). The sample was centrifuged at 13,200 rpm for 2 min, the sample vial was removed, and sample centrifuged again for another 2 minutes. The filtrate was transferred to a $500 \mu \mathrm{L}$ microcentrifuge tube and electrokinetic injections were made by placing the end of the separation capillary into this tube. Fly samples and standard samples made in the water and perchloric acid sample matrix were stored on ice in the dark until use to minimize reduce sample degradation.

\subsubsection{Instrumentation and Data Analysis}

The CE with end column FSCV was built in house and was performed as previously described (see Supporting Information). ${ }^{17}$

Fast-scan cyclic voltammetry was performed as previously described. ${ }^{17}$ Quantitation of analytes in fly samples was performed via post calibration using a $10 \mu \mathrm{L}$ (0.5 mM perchloric acid) solution with $100 \mathrm{nM}$ of each standard mixture of dopamine, tyramine, octopamine, and serotonin. The response (in $n A$ ) of the electrode to the known concentration of the standard mix was used to determine the concentration of analytes in the fly sample. All CVs were filtered at $20 \mathrm{kHz}$ to remove noise. 


\subsubsection{Statistics}

Error bars are shown as standard error of the mean (SEM). Statistics were performed in GraphPad Prism 6 (La Jolla, CA). Unpaired t-tests were used to compare means from two groups. One-way ANOVA with Bonferroni post-tests were used to compare three or more groups. Outlier testing was performed on all data sets using $\operatorname{ROUT}(\mathrm{Q}=0.1 \%)$, and definitive outliers were removed.

\subsubsection{Acknowledgments.}

This work was funded by NIH (MH1R01NS076875) to BJV.

\subsubsection{References}

1. Denno, M. E., Privman, E., and Venton, B. J. (2015) Analysis of Neurotransmitter Tissue Content of Drosophila melanogaster in Different Life Stages. ACS Chem. Neurosci. 6, 117-123.

2. Shaver, S. A., Riedl, C. A. L., Parkes, T. L., Sokolowski, M. B., and Hilliker, A. J. (2000) Isolation of Larval Behavioral Mutants in Drosophila Melanogaster. Journal of Neurogenetics 14, 193-205.

3. Urbach, R., Schnabel, R., and Technau, G. M. (2003) The pattern of neuroblast formation, mitotic domains and proneural gene expression during early brain development in Drosophila. Development 130, 3589-3606.

4. Lichtneckert, R., Bello, B., and Reichert, H. (2007) Cell lineage-specific expression and function of the empty spiracles gene in adult brain development of Drosophila melanogaster. Development 134, 1291-1300.

5. Robinow, S., Talbot, W. S., Hogness, D. S., and Truman, J. W. (1993) Programmed cell death in the Drosophila CNS is ecdysone-regulated and coupled with a specific ecdysone receptor isoform. Development 119, 1251-1259.

6. Truman, J. W., Bate, M. (1988) Spatial and temporal patterns of neurogenesis in the central nervous system of Drosophila melanogaster. Dev. Biol. 125, 145-157.

7. Corey, J. L., Quick, M. W., Davidson, N., Lester, H. A., and Guastella, J. (1994) A cocaine-sensitive Drosophila serotonin transporter: cloning, expression, and electrophysiological characterization. PNAS 91, 1188-1192. 
8. Pörzgen, P., Park, S. K., Hirsh, J., Sonders, M. S., and Amara, S. G. (2001) The Antidepressant-Sensitive Dopamine Transporter in Drosophila melanogaster: A Primordial Carrier for Catecholamines. Mol. Pharmacol. 59, 83-95.

9. Roeder, T. (2004) Tyramine and Octopamine: Ruling Behavior and Metabolism. Annu. Rev. Entomol. 50, 447-477.

10. Vickrey, T. L., Condron, B., and Venton, B. J. (2009) Detection of Endogenous Dopamine Changes in Drosophila melanogaster Using Fast-Scan Cyclic Voltammetry. Anal. Chem. 81, 9306-9313.

11. Borue, X., Condron, B., and Venton, B. J. (2010) Both synthesis and reuptake are critical for replenishing the releasable serotonin pool in Drosophila. $J$. Neurochem. 113, 188-199.

12. Chang, H. Y., Grygoruk, A., Brooks, E. S., Ackerson, L. C., Maidment, N. T., Bainton, R. J., and Krantz, D. E. (2005) Overexpression of the Drosophila vesicular monoamine transporter increases motor activity and courtship but decreases the behavioral response to cocaine. Mol Psychiatry 11, 99-113.

13. Chen, A., Ng, F., Lebestky, T., Grygoruk, A., Djapri, C., Lawal, H. O., Zaveri, H. A., Mehanzel, F., Najibi, R., Seidman, G., Murphy, N. P., Kelly, R. L., Ackerson, L. C., Maidment, N. T., Jackson, F. R., and Krantz, D. E. (2013) Dispensable, Redundant, Complementary, and Cooperative Roles of Dopamine, Octopamine, and Serotonin in Drosophila melanogaster. Genetics 193, 159-176.

14. Kuklinski, N. J., Berglund, E. C., Engelbreksson, J., and Ewing, A. G. (2010) Determination of salsolinol, norsalsolinol, and twenty-one biogenic amines using micellar electrokinetic capillary chromatography-electrochemical detection. ELECTROPHORESIS 31, 1886-1893.

15. Powell, P. R., Paxon, T. L., Han, K. A., and Ewing, A. G. (2005) Analysis of Biogenic Amine Variability among Individual Fly Heads with Micellar Electrokinetic Capillary Chromatography-Electrochemical Detection. Anal. Chem. 77, 6902-6908.

16. Fang, H., Pajski, M. L., Ross, A. E., and Venton, B. J. (2013) Quantitation of dopamine, serotonin and adenosine content in a tissue punch from a brain slice using capillary electrophoresis with fast-scan cyclic voltammetry detection. Anal. Methods 5, 2704-2711.

17. Fang, H., Vickrey, T. L., and Venton, B. J. (2011) Analysis of Biogenic Amines in a Single Drosophila Larva Brain by Capillary Electrophoresis with Fast-Scan Cyclic Voltammetry Detection. Anal. Chem. 83, 2258-2264.

18. Zhang, C. X., Thormann, W. (1996) Head-Column Field-Amplified Sample Stacking in Binary System Capillary Electrophoresis: A Robust Approach Providing over 1000-Fold Sensitivity Enhancement. Anal. Chem. 68, 2523-2532. 
19. Weng, Q., Xu, G., Yuan, K., and Tang, P. (2006) Determination of monoamines in urine by capillary electrophoresis with field-amplified sample stacking and amperometric detection. J. Chromatogr. B 835, 55-61.

20. Sánchez-Rivera, A. E., Corona-Avendaño, S., Alarcón-Angeles, G., RojasHernández, A., Ramírez-Silva, M. T., and Romero-Romo, M. A. (2003) Spectrophotometric study on the stability of dopamine and the determination of its acidity constants. Spectrochim. Acta A 59, 3193-3203.

21. Monastirioti, M., Linn, J., and White, K. (1996) Characterization of Drosophila Tyramine b-Hydroxylase Gene and Isolation of Mutant Flies Lacking Octopamine. The Journal of Neuroscience 16, 3900-3911.

22. Kuklinski, N. J., Berglund, E. C., Engelbrektsson, J., and Ewing, A. G. (2010) Biogenic Amines in Microdissected Brain Regions of Drosophila melanogaster Measured with Micellar Electrokinetic Capillary Chromatography-Electrochemical Detection. Anal. Chem. 82, 7729-7735.

23. Hardie, S. L., Hirsh, J. (2006) An improved method for the separation and detection of biogenic amines in adult Drosophila brain extracts by high performance liquid chromatography. J. Neurosci. Methods 153, 243-249.

24. Dasari, S., Viele, K., Turner, A. C., and Cooper, R. L. (2007) Influence of PCPA and MDMA (ecstasy) on physiology, development and behavior in Drosophila melanogaster. Eur. J. Neurosci. 26, 424-438.

25. Yarali, A., Krischke, M., Michels, B., Saumweber, T., Mueller, M. J., and Gerber, B. (2009) Genetic Distortion of the Balance between Punishment and Relief Learning in Drosophila. J. Neurogenet. 23, 235-247.

26. Monastirioti, M., Gorczyca, M., Rapus, J., Eckert, M., White, K., and Budnik, V. (1995) Octopamine immunoreactivity in the fruit fly Drosophila melanogaster. J. Comp. Neurol. 356, 275-287.

27. Saraswati, S., Fox, L. E., Soll, D. R., and Wu, C. F. (2004) Tyramine and octopamine have opposite effects on the locomotion of Drosophila larvae. J. Neurobiol. 58, 425-441.

28. St.Pierre, S. E., Ponting, L., Stefancsik, R., McQuilton, P., and the FlyBase Consortium (2014) FlyBase102- advanced approaches to interrogating FlyBase. Nucleic Acids Res. 42, D780-D788.

29. Mustard, J. A., Kurshan, P. T., Hamilton, I. S., Blenau, W., and Mercer, A. R. (2005) Developmental expression of a tyramine receptor gene in the brain of the honey bee, Apis mellifera. J. Comp. Neurol. 483, 66-75.

30. Ohhara, Y., Kayashima, Y., Hayashi, Y., Kobayashi, S., and YamakawaKobayashi, K. (2012) Expression of b-adrenergic-like Octopamine Receptors during Drosophila Development. Zool. Sci. 29, 83-89. 
31. Walker, Q. D., Rooney, M. B., Wightman, R. M., and Kuhn, C. M. (1999) Dopamine release and uptake are greater in female than male rat striatum as measured by fast cyclic voltammetry. Neuroscience 95, 1061-1070.

32. Geng, C., Sparks, T. C., Skomp, J. R., and Gajewski, R. P. (1993) Biogenic amines in the brain of Manduca sexta during larval-pupal metamorphosis. Comp. Biochem. Physiol. , Part C: Pharmacol. , Toxicol. Endocrinol. 106, 275-284.

33. Liu, T., Dartevelle, L., Yuan, C., Wei, H., Wang, Y., Ferveur, J. F., and Guo, A. (2008) Increased Dopamine Level Enhances Male-Male Courtship in Drosophila. The Journal of Neuroscience 28, 5539-5546.

34. Neckameyer, W. S. (1998) Dopamine Modulates Female Sexual Receptivity in Drosophila Melanogaster. J. Neurogenet. 12, 101-114.

35. Wicker-Thomas, C., Hamann, M. (2008) Interaction of dopamine, female pheromones, locomotion and sex behavior in Drosophila melanogaster. $J$. Insect Physiol. 54, 1423-1431.

36. Drossopoulou, G., Antoniou, K., Kitraki, E., Papathanasiou, G., Papalexi, E., Dalla, C., and Papadopoulou-Daifoti, Z. (2004) Sex differences in behavioral, neurochemical and neuroendocrine effects induced by the forced swim test in rats. Neuroscience 126, 849-857.

37. Kennett, G. A., Chaouloff, F., Marcou, M., and Curzon, G. (1986) Female rats are more vulnerable than males in an animal model of depression: the possible role of serotonin. Brain Res. 382, 416-421.

38. Cosgrove, K. P., Mazure, C. M., and Staley, J. K. (2007) Evolving Knowledge of Sex Differences in Brain Structure, Function, and Chemistry. Biol. Psychiatry 62, 847-855.

39. Svetec, N., Ferveur, J. F. (2005) Social experience and pheromonal perception can change male-male interactions in Drosophila melanogaster. J. Exp. Biol. $208,891-898$.

40. Zhou, C., Rao, Y., and Rao, Y. (2008) A subset of octopaminergic neurons are important for Drosophila aggression. Nat Neurosci 11, 1059-1067.

41. Hoyer, S. C., Eckart, A., Herrel, A., Zars, T., Fischer, S. A., Hardie, S. L., and Heisenberg, M. (2008) Octopamine in Male Aggression of Drosophila. Curr. Biol. 18, 159-167.

42. Dierick, H. A., Greenspan, R. J. (2007) Serotonin and neuropeptide F have opposite modulatory effects on fly aggression. Nat Genet 39, 678-682. 
Chapter 3. Quantification of histamine and carcinine in Drosophila melanogaster tissues 


\subsection{Abstract:}

Histamine is a neurotransmitter crucial to the visual processing of Drosophila melanogaster. It is inactivated by metabolism to carcinine, a $\beta$-alanyl derivative, and the same enzyme that controls that process also converts dopamine to $\mathrm{N}$ - $\beta$-alanyl dopamine. Direct detection of histamine and carcinine has not been reported in single Drosophila brains. In this chapter we quantify histamine, carcinine, dopamine, and N- $\beta$ alanyl dopamine in Drosophila tissues by capillary electrophoresis coupled to fast-scan cyclic voltammetry (CE-FSCV). Limits of detection were low, $4 \pm 1 \mathrm{pg}$ for histamine, $10 \pm$ $4 \mathrm{pg}$ for carcinine, $2.8 \pm 0.3 \mathrm{pg}$ for dopamine, and $9 \pm 3 \mathrm{pg}$ for $\mathrm{N}$ - $\beta$-alanyl-dopamine. Tissue content was compared in the brain, eyes, and cuticle from wild type (Canton S) and mutant $\left(\tan ^{3}\right.$ and ebony $\left.{ }^{1}\right)$ strains. In $\tan ^{3}$ mutants, the enzyme that produces histamine from carcinine is non-functional while in ebony ${ }^{1}$ mutants, the enzyme that produces carcinine from histamine is non-functional. In all fly strains, the neurotransmitter content was highest in the eyes and there were no strain differences for tissue content in the cuticle. The main finding was that carcinine levels changed significantly in the mutant flies while histamine levels did not. In particular, $\tan ^{3}$ flies had significantly higher carcinine levels in the eyes and brain than Canton S or ebony ${ }^{1}$ flies. $\mathrm{N}$ - $\beta$-alanyl-dopamine was detected in $\tan ^{3}$ mutants, but not in other strains. These results show the utility of CE-FSCV for sensitive detection of histamine and carcinine which allows a better understanding of their content and metabolism in different types of tissues. This work was published in ACS Chem. Neurosci., 2016, 7 (3), pp 407-414. ${ }^{1}$ 


\subsection{Introduction:}

Histamine is an important neurotransmitter in both Drosophila and humans. In mammals and flies, it functions to promote wakefulness and circadian rhythm regulation. ${ }^{2,3}$ Histamine, and not glutamate, is the major photoreceptor transmitter in Drosophila. ${ }^{4,5}$ Histamine is synthesized from histidine by histidine decarboxylase (hdc). When this enzyme is non-functional $(h d c)$, flies are blind and behaviorally abnormal ${ }^{6}$, demonstrating the necessity of histamine for normal functioning of the visual system. In the brain and visual system of Drosophila, tight control of histamine content is necessary for the visual transduction process, and part of this control occurs through the metabolism of histamine to carcinine, which is "inactive". ${ }^{7}$ Glial cells typically uptake histamine and then convert it to carcinine, which is $\beta$-alanyl histamine. ${ }^{8}$ The enzyme for this conversion, $\mathrm{N}$ - $\beta$-alanyl-dopamine synthase (also called Ebony), also converts dopamine, and several other biogenic amines, into a $\beta$-alanyl derivative. ${ }^{9}$ Conversion of dopamine to $\mathrm{N}$ - $\beta$-alanyl dopamine is important for cuticle scleritization and pigmentation; mutants deficient in $\mathrm{N}$ - $\beta$-alanyl-dopamine synthase appear dark (ebony) and also exhibit an altered visual processing phenotype. ${ }^{10}$ Conversion of carcinine back to histamine is also important for regulating the amount of histamine available for neurotransmission, as the rate of histamine synthesis is slow. The enzyme $\beta$-alanyl-dopamine hydrolase (also called Tan) converts carcinine to histamine and N- $\beta$-alanyl dopamine to dopamine. Deficiency in $\beta$-alanyl-dopamine hydrolase also produces a phenotype that is visible in the cuticle (a light tan color) in addition to visual defects. ${ }^{11}$ Furthermore, these mutations also influence the behavior of the flies, ${ }^{12}$ causing abnormal locomotor activity rhythms. We hypothesize that tan and ebony mutants will have altered content of histamine, carcinine, and N- $\beta$-alanyl-dopamine, but levels of these neurotransmitters have not been explored in different tissues because of a lack of good analytical technique 
Tissue content of histamine in Drosophila has typically been measured using high performance liquid chromatography (HPLC). Borycz and co-workers reported tissue content determination from pooled whole heads using HPLC coupled to electrochemical detection (amperometry) in samples treated with o-phthaldialdehyde and mercaptoethanol to increase sensitivity; histamine is also natively electroactive, and does not require treatment to be detected using electrochemical detectors. ${ }^{13-15}$ However, they reported difficulty detecting carcinine using this method, and later carcinine quantification was performed by titrating $\left[{ }^{3} \mathrm{H}\right]$ histamine. ${ }^{16}$ These papers used samples of pooled whole heads, ${ }^{11}$ likely due to the low concentrations of histamine present in the fly samples. Given the presence of histamine and carcinine in the eye and the brain, and its possible presence in cuticle (along with dopamine and $\mathrm{N}$ - $\beta$-alanyl-dopamine), quantitation in specific tissues is necessary to better understand the localization of these neurotransmitters and their metabolites in Drosophila. We have demonstrated the utility of capillary electrophoresis coupled to fast scan cyclic voltammetry (CE-FSCV) for the separation and quantification of the monoamines dopamine, serotonin, octopamine and tyramine from single ventral nerve cords from larvae ${ }^{17}$ and brains from adult Drosophila. ${ }^{18}$ Capillary electrophoresis with field amplified sample stacking provides high sensitivity and uses small sample volumes, reducing the need for large pooled samples.

In this study, we optimized parameters for CE-FSCV to separate and quantify histamine, carcinine, dopamine, and $\beta$-alanyl-dopamine in tissue from Drosophila melanogaster. With this technique, we can measure neurotransmitters separately from the brain, eyes, or cuticle of a single fly. The content of all three tissue types was analyzed in a wild type fly, Canton S, and in two mutants, $\tan ^{3}$ and ebony ${ }^{1}$, who are expected to have altered tissue content of histamine and carcinine. Eyes contained the highest levels of histamine and carcinine, followed by the cuticle and then the brain. Both of the mutants showed differences in tissue content of carcinine in the brain and eyes 
but not histamine. Ratios of histamine to carcinine were significantly altered in the brain and in the eyes in both the ebony ${ }^{1}$ and $\tan ^{3}$ mutants. $\beta$-alanyl-dopamine was only detected in the cuticle and eyes of $\tan ^{3}$. Thus, Ebony and Tan are important for the metabolic production and inactivation of histamine, and mutating their function produces different ratios of histamine to car. In addition, Tan is important for the metabolism of dopamine outside of the brain.

\subsection{Results and Discussion}

\subsubsection{Capillary Electrophoresis with Fast Scan Cyclic Voltammetry for histamine and carcinine detection}

Capillary electrophoresis coupled to fast-scan cyclic voltammetry (CE-FSCV) can be used to analyze single brains from Drosophila melanogaster. ${ }^{18,17}$ Many neurotransmitters, including histamine, and their metabolites are electroactive and are detected by FSCV using carbon-fiber microelectrodes. ${ }^{13,14,19}$ In this study, we expanded the scope of CE-FSCV to include three new analytes: histamine (HA), carcinine (CA), and $\mathrm{N}$ - $\beta$-alanyl- dopamine (BADA). Histamine and carcinine were chosen because of their importance in the visual process of Drosophila ${ }^{4,7}$, and N- $\beta$-alanyl-dopamine was analyzed because it shares a synthesis pathway with carcinine. ${ }^{20,11}$ These biogenic amines are found in a variety of tissues, so brains, eyes, and cuticle were studied. In order to detect both histamine and carcinine, the electrode was scanned from $-0.4 \mathrm{~V}$ to $1.4 \mathrm{~V}$ at a rate of $400 \mathrm{~V} / \mathrm{s}(10 \mathrm{~Hz}$ repetition rate); this waveform provides high sensitivity for a wide variety of analytes ${ }^{18}$ and the higher switching potential is required for sensitive detection of both histamine and carcinine..$^{21,22}$ Using this waveform, limits of detection for the analytes in $10 \mu \mathrm{L}$ of $0.5 \mathrm{mM}$ perchloric acid were $4 \pm 1 \mathrm{pg}$ for histamine, $10 \pm 4 \mathrm{pg}$ for carcinine, $2.8 \pm 0.3 \mathrm{pg}$ for dopamine and $9 \pm 3 \mathrm{pg}$ for $\mathrm{N}$ - $\beta$-alanyl-dopamine. 
The separation buffer used for histamine, carcinine, dopamine, and N- $\beta$-alanyldopamine was modified from the buffer previously used for larval samples. ${ }^{17} \mathrm{~A} \mathrm{pH} 2,200$

A.

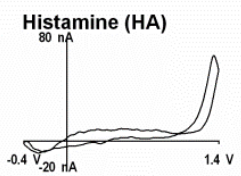

$\underset{40 \mathrm{nA}}{\operatorname{Carcinine}}(\mathrm{CA})$

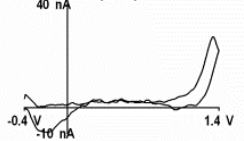

Dopamine (DA)

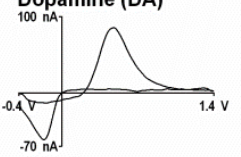

B.

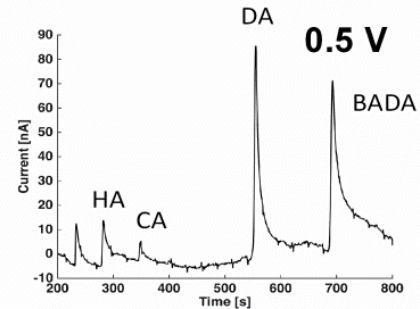

C.

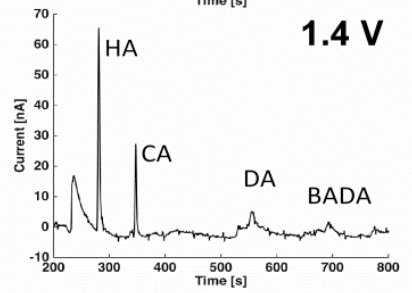

D.

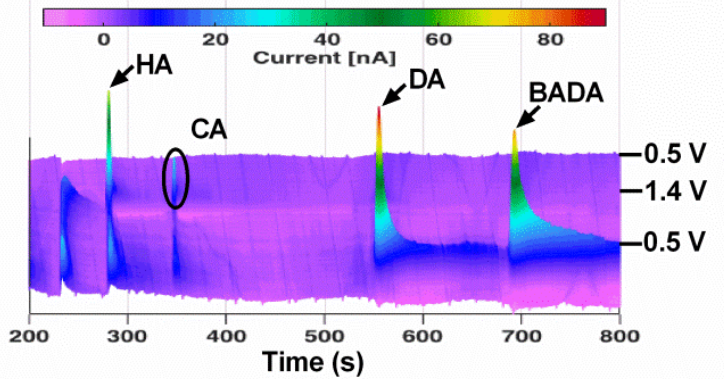

Figure 3-1 Separations of a standard mixture of neurotransmitters: $100 \mathrm{nM}$ each of histamine (HA), carcinine (CA), dopamine (DA), and $N$ - $\beta$-alanyldopamine (BADA) in $10 \mu \mathrm{L}$ of $0.5 \mathrm{mM}$ perchloric acid. A. Cyclic voltammograms of the analytes. $B$.

Electropherogram at $0.5 \mathrm{~V}$; DA and BADA are most visible at this potential. C. Electropherogram at $1.4 \mathrm{~V}$ for the same separation as above; $\mathrm{HA}$ and $\mathrm{CA}$ are most visible at this potential. D. A heat map showing the migrations of histamine, carcinine (circled, in black), dopamine, and N$\beta$-alanyl-dopamine. Adapted (with permission) from Denno et al. 2016.
mM phosphate buffer was used.

Preliminary experiments showed

that this lower $\mathrm{pH}$ was important, as

migration times for histamine and

carcinine were irreproducible at $\mathrm{pH}$

4.0, likely due to wall interactions. At

$\mathrm{pH}<3.0$, wall interactions are

greatly reduced as silanol groups

are fully protonated and do not react

with protonated amines. Fewer

silanol groups means that

electroosmotic flow is largely

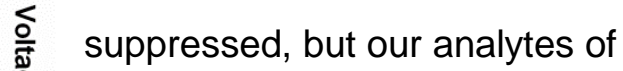

interest are all protonated and

migrate towards the anode. Due to

EOF suppression, wall coatings or

added buffer modifiers are not

needed, thus simplifying the

separation. ${ }^{23}$ All analytes appeared

in 800 seconds and (approximately

13.3 minutes) were well resolved

from one another (Figure 1). 
With CE-FSCV, characteristic cyclic voltammograms are obtained for electroactive species present in the sample (Figure $1 \mathrm{~A}$ ); these voltammograms are used to confirm the identity of analytes and for quantification of analytes. ${ }^{17}$ Due to the high sampling frequency, a large number of cyclic voltammograms are collected during a separation, and these can be represented in a heat map. The heat map plots applied potential on the $y$ axis, time on the $\mathrm{x}$ axis, and the current as a peak of color, which allows for easy visualization of all electroactive species in the separation (Figure 1B). The heat map only displays the oxidation peaks for the sake of clarity, however the reduction peaks are there and can be seen on the cyclic voltammograms (Figure $1 \mathrm{~A}$ ). From this heat map, one can visualize that histamine and carcinine migrate first and have higher oxidation

potentials than dopamine and $\mathrm{N}$ - $\beta$-alanyl dopamine. In

contrast, current vs. time
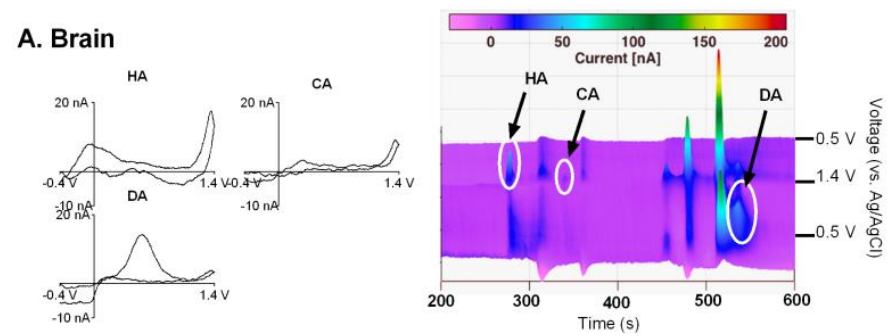

traces (Figure $1 \mathrm{C}$ and $\mathrm{D}$ ) only show the current at a given potential, which means not every analyte is visible on every trace. Histamine and carcinine are visible at higher potentials (1.4 V, Figure 1C), and dopamine and $\mathrm{N}-\beta$-alanyl-
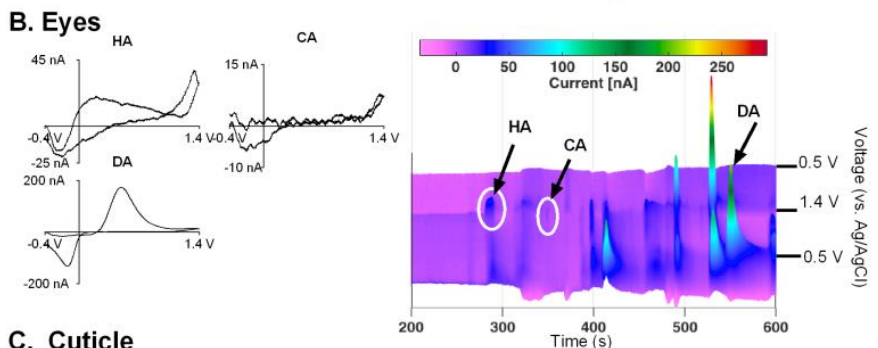

C. Cuticle

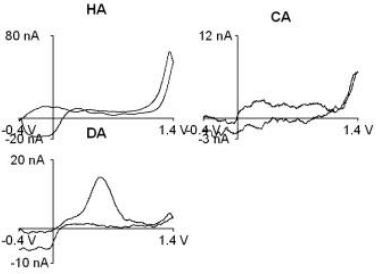

dopamine are visible at lower

potentials (0.5 V, Figure 1D).

Figure 3-2 Separations of histamine, carcinine, and dopamine from three tissue types. A. Brain B. Eyes and C. Cuticle of a single Canton $\mathrm{S}$ fly. The cyclic voltammograms shown above confirm the identity of the analytes. The heat maps below show the separation of these analytes in different tissues. The peaks are circled and labeled to show their position. Adapted (with permission) from Denno et al. 2016. 
Separations were performed of biogenic amines in the three different tissue types taken from a wild type fly (Canton S). In each case, samples from a single adult fly were analyzed; for eye samples, both eyes from a single fly were combined. Histamine, carcinine, and dopamine were found in all of the tissue types tested (Figure 2). However, no N- $\beta$-alanyl-dopamine was detected in any of the Canton S samples; thus, the amount of $\mathrm{N}$ - $\beta$-alanyl-dopamine present in these tissues is below our limit of detection. The heat maps of the brain (Figure 2A), the eyes (Figure 2B), and the cuticle (Figure 2C) show that these tissues contain different amounts of histamine, carcinine, and dopamine. In the heat map for brain tissue (Fig. 2A), histamine and carcinine are clearly visible. While dopamine is harder to see in the heat map because of an unknown electroactive peak, it is separated from the interferent and can be distinguished by looking at the cyclic voltammograms (Fig. $2 \mathrm{~A}, \mathrm{C}$ ). In the eye (Fig. 2B), there is more carcinine than in the brain and more dopamine than in either the cuticle or the brain. In the cuticle (Fig. 2C), the heat map shows a high amount of histamine and a smaller amount of carcinine and dopamine. While there are other unidentified electroactive compounds in these tissue samples that appear on the heat maps, they do not interfere with detection of our analytes of interest (Figure 2A, Figure 2C). Migration times for these separations are consistent, even though they were obtained on different days.

\subsubsection{Quantification of Histamine, Carcinine, and Dopamine in the Brain, Eyes, and Cuticle of Drosophila \\ Using the CE-FSCV method, adult brains, eyes, and cuticle of three strains of} Drosophila were compared to determine the content of histamine, 
carcinine, and dopamine.

Canton S, a widely studied wild-type strain with normal metabolism of histamine, carcinine, and dopamine was used as a control. Two strains of flies with non-functional enzymes for the synthesis of carcinine $\left(e b o n y^{1}\right)$ and the metabolism of carcinine $\left(\tan ^{3}\right)$ (Scheme 1) were selected to study the impact of

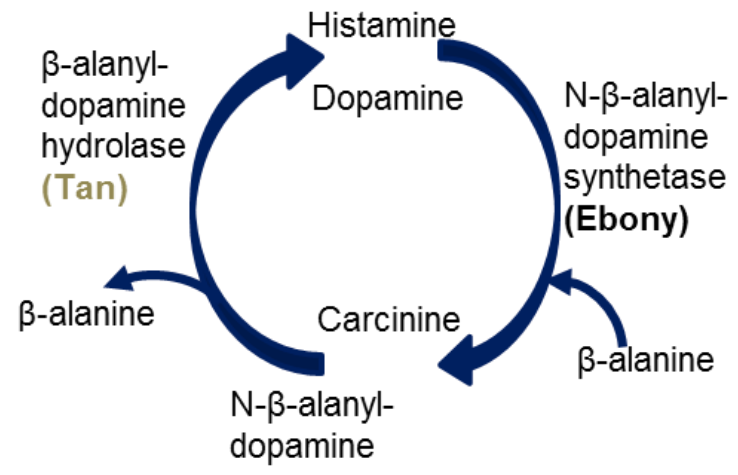

Scheme 3-1 A simplified schematic of histamine metabolism. Histamine and dopamine are metabolized by $\mathrm{N}$ - $\beta$-alanyl-dopamine synthetase (Ebony) into their $\beta$ alanyl derivatives, carcinine and $\mathrm{N}$ - $\beta$-alanyl-dopamine. These metabolites can then be returned to histamine and carcinine by $\beta$-alanyl-dopamine hydrolase (Tan). Adapted (with permission) from Denno et al. 2016.

mutations in enzymes on tissue content of histamine and carcinine. Ebony ${ }^{1}$ mutants lack $\mathrm{N}$ - $\beta$-alanyl dopamine synthetase (also called Ebony) which is responsible for the metabolism of histamine and dopamine to carcinine and $\mathrm{N}$ - $\beta$-alanyl dopamine, respectively. ${ }^{16,7} \operatorname{Tan}^{3}$ mutants lack $\beta$-alanyl-dopamine hydrolase (also called Tan), which metabolizes carcinine and $\mathrm{N}$ - $\beta$-alanyl dopamine to histamine and dopamine, respectively (Scheme 1). ${ }^{16,11}$

Tissue content was first compared in the different tissue types from single flies. Because different tissue samples had different masses, the amount of neurotransmitter was divided by the average weight of the tissue type to give pg neurotransmitter/mg tissue (Table 1). Overall, the tissue content of neurotransmitters was highest in the eyes, followed by the brain and then the cuticle. For histamine, there was a main effect of tissue type (two-way ANOVA, $p<0.0001$ ) but no effect of genotype. Histamine content was significantly higher in the eyes than either the brain or the cuticle (Bonferonni post test $p=0.0009$ and $p=0.0003$, respectively) in Canton $S$ and higher in the eye than the cuticle $(p=0.0372)$ in ebony ${ }^{1}$. There were no differences in histamine content among tissue types for $\tan ^{3}$ ( $p>0.05$ for all tissues). Carcinine had a much different pattern than 
histamine, with large

differences especially in

the $\tan ^{3}$ flies. For carcinine,

there was a main effect of

genotype (two-way

ANOVA, $p=0.0004)$ and

tissue type $(p=0.0012)$,

with a significant

interaction between these

factors $(p=0.0006)$. In

Canton S or ebony ${ }^{1}$ flies,

tissue content of carcinine

did not significantly differ in
Table 3-1: Tissue content per milligram of tissue of histamine, carcinine, and dopamine in brain, eyes, and cuticle.

\begin{tabular}{|l|l|l|l|}
\hline \multicolumn{5}{|c|}{ Canton S } \\
\hline Tissue type & Histamine & Carcinine & Dopamine \\
\hline Brain $(n=7)$ & $1250 \pm 400$ & $1650 \pm 400$ & $1100 \pm 200$ \\
\hline Eyes $(n=7)$ & $12400 \pm 3600$ & $5460 \pm 1600$ & $4300 \pm 2000$ \\
\hline Cuticle $(n=7)$ & $390 \pm 150$ & $660 \pm 300$ & $83 \pm 30$ \\
\hline \multicolumn{5}{|c|}{ tan $^{3}$} \\
\hline Tissue type & Histamine & Carcinine & Dopamine \\
\hline Brain $(n=5)$ & $750 \pm 50$ & $9350 \pm 2700$ & $4960 \pm 1900$ \\
\hline Eyes $(n=7)$ & $7700 \pm 4000$ & $58700 \pm 9000$ & $23600 \pm 1000$ \\
\hline $\begin{array}{l}\text { Cuticle }(n= \\
5)\end{array}$ & $290 \pm 100$ & $920 \pm 400$ & $690 \pm 300$ \\
\hline \multicolumn{5}{|c|}{ ebony } \\
\hline Tissue type & Histamine & Carcinine & Dopamine \\
\hline Brain $(n=5)$ & $1010 \pm 200$ & $1150 \pm 400$ & $1760 \pm 1000$ \\
\hline Eyes $(n=8)$ & $7720 \pm 2000$ & $1200 \pm 300$ & $1490 \pm 400$ \\
\hline $\begin{array}{l}\text { Cuticle }(n= \\
6)\end{array}$ & $230 \pm 90$ & $190 \pm 90$ & $60 \pm 20$ \\
\hline
\end{tabular}

All tissue contents are pg neurotransmitter/mg tissue.

any of the tissue types. However, in $\tan ^{3}$ flies carcinine content in the eyes was significantly higher than the brain or the cuticle respectively (Bonferoni post-test, $p<$ 0.0001 for both). Dopamine also varied by tissue type and genotype (two-way ANOVA, $p=0.0251$ and $p=0.0311$ respectively), but there was no significant interaction between these variables. There were no significant differences among tissue content of dopamine in any of the Canton S or ebony ${ }^{1}$ tissues. For $\tan ^{3}$, there was significantly more dopamine in the eye than in the cuticle or the brain ( $p=0.0019$ and 0.0136 respectively). 
Differences in neurotransmitter distribution among tissue types are likely due to differing gene expression levels for the enzymes controlling the synthesis of these

neurotransmitters and

metabolites. For example, the

expression of Ebony is highest in

the eye, followed by the brain. ${ }^{24}$

Similarly, the expression of Tan is

highest in the eye, followed by the

brain. ${ }^{24}$ In both cases, there is

also evidence of expression in the

cuticle, due to the phenotypic

effect of mutations to either gene.

The eyes have the highest

content of neurotransmitter for all

three neurotransmitters,

indicating large pools that are

used for visual processing. In

contrast, the cuticle has the

smallest amounts, which is not

surprising since this is not where

neuronal communication is taking

place.

Another way to compare

the data is to look at each tissue

type and compare the effects of
A. Brain

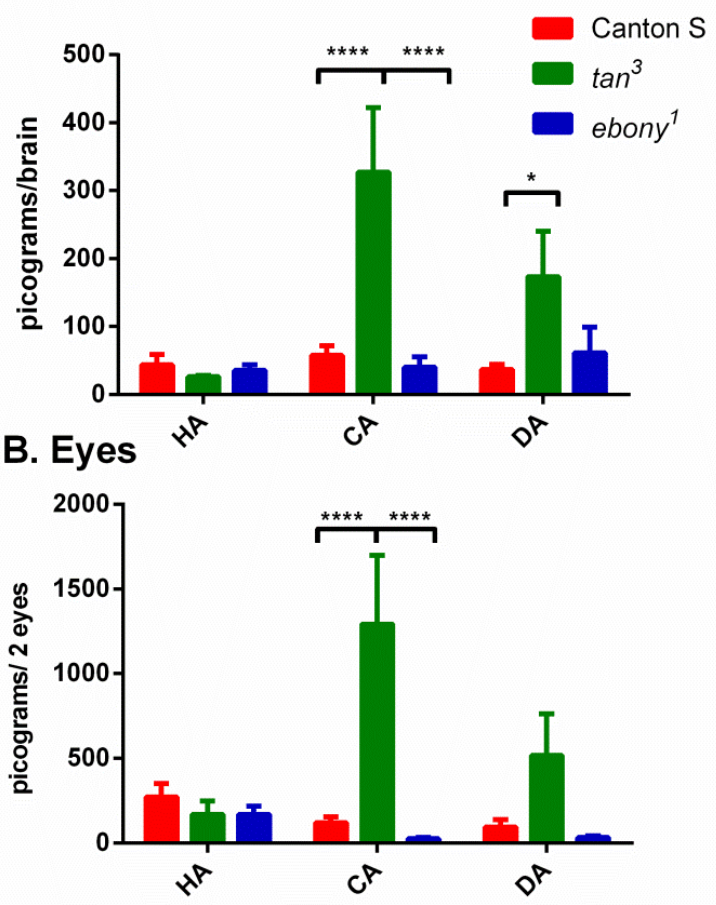

C. Cuticle

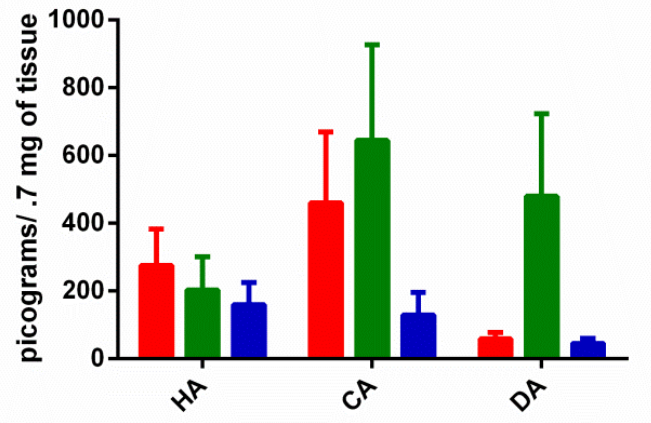

Figure 3-3 Comparisons of tissue content in each tissue type for all strains. A. Brain ( $n=5-7)$ B. Eyes $(n=7-8)$ C.

Cuticle ( $n=5-7)$. Two-way ANOVA and Bonferroni post tests were used to compare the tissue content of each neurotransmitter. A. In brain, there was a significant effect of genotype $(p=0.0025)$ and neurotransmitter $(p=0.0048)$ and a significant interaction (two-way ANOVA, $p=0.0025)$. B. In eyes, there was a significant effect of genotype $(p<0.0001)$ and a significant interaction between neurotransmitter and genotype $(p=0.0009)$. C. In cuticle, there is a significant effect of genotype (two way ANOVA, $p=0.0347$ ) but not neurotransmitter. ${ }^{*} p<0.05,{ }^{* * * *} p<0.0001$. Adapted (with permission) from Denno et al. 2016. 
genotype and type of neurotransmitter. Because the mass would be the same for each sample, we compared pg of

neurotransmitter instead of $\mathrm{pg} / \mathrm{mg}$ (Fig.

3). Overall, the main finding is that

histamine does not vary much with the

different genotypes but carcinine varies

dramatically. In the brain (Figure 3A),

there was a significant main effect of

genotype (two-way ANOVA, $p=0.0001$ )

and neurotransmitter $(p=0.0048)$ and a

significant interaction between these

variables $(p=0.0025)$. While there was

no difference for histamine between any

of the genotypes in the brain, carcinine

was significantly higher in the $\tan ^{3}$ brain

than in the Canton S or ebony ${ }^{1}$ brains

(Bonferroni post test, $p<0.0001$ for

both). $\operatorname{Tan}^{3}$ also had increased dopamine

compared to Canton S brains

$(p=0.0333)$. In the eyes (Figure 3B), the

greatest effect of genotype was again on

carcinine content and not histamine

content; $\tan ^{3}$ flies again showed the

A. Brain

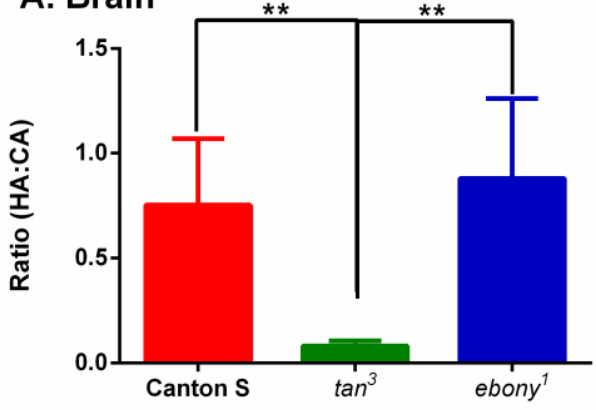

B. Eyes

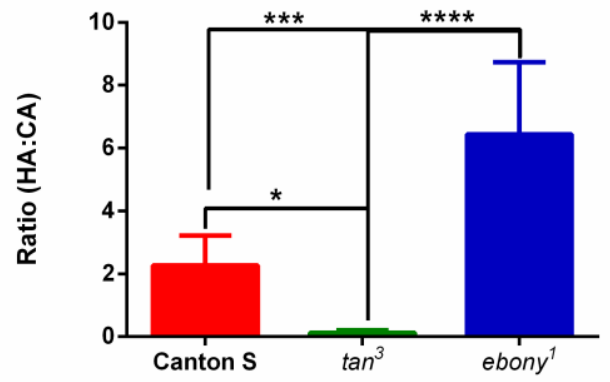

C. Cuticle

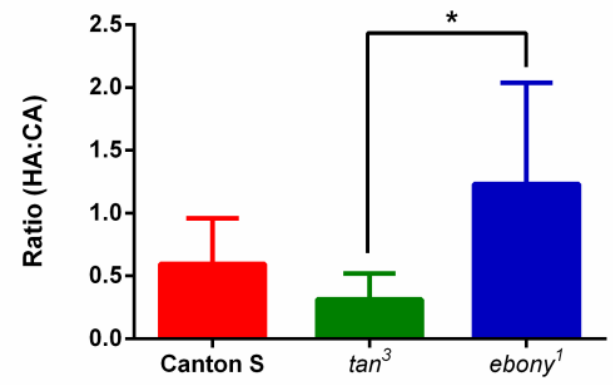

Figure 3-4: Comparisons of ratios of histamine to carcinine ratios (HA:CA) for all three tissue types. A. Brain $(n=5-7)$ B. Eyes $(n=7-8)$ C. Cuticle $(n=5-7)$. One-way ANOVA with Bonferroni post test was used to compare these ratios. A. In the brain, there was a significant effect of genotype $(p=$ $0.0012)$. B. In the eyes, there was a significant effect of genotype $(p<0.0001)$. C. In the cuticle, there was a significant effect of genotype. ${ }^{*} p<0.05,{ }^{* \star} p<0.01$, ${ }^{* * *} p<0.001,{ }^{* * * *} p<0.0001$. Adapted (with permission) from Denno et al. 2016. largest differences in carcinine content.

Overall, there was a significant effect of genotype in the eyes (Two-way ANOVA, $p<$ $0.0001)$ but no significant effect of neurotransmitter $(p=0.0603)$. There was also a 
significant interaction between genotype and neurotransmitter in the eyes (Two-way ANOVA, $p=0.0009)$. There were no significant differences in histamine or dopamine content in eyes between the strains of flies, but carcinine content was significantly higher in $\tan ^{3}$ eyes than in either Canton $S$ or ebony ${ }^{1}$ eyes $(p<0.0001$ for both). In the cuticle (Figure 3C), there was a significant effect of genotype (two-way ANOVA, $p=0.0347$ ). However, Bonferroni post tests showed that there were no significant differences in any neurotransmitters in any of the tested strains.

Another way of analyzing the differences between the strains of flies is to compare the ratio of histamine to carcinine (HA:CA, Figure 4). This ratio provides information on the balance between histamine and carcinine and how this is altered by mutations in the metabolic cycle such as tan and ebony. In the brain (Figure 4A), there was a main effect of genotype on the HA:CA ratio (one-way ANOVA, $p=0.0012$ ) and ebony ${ }^{1}$ brains had a significantly higher ratio than the $\tan ^{3}$ brains (Bonferroni post test, $\mathrm{p}=0.0021)$. Additionally, the HA:CA ratio in Canton $\mathrm{S}$ brains was higher than in $\tan ^{3}$ brains $(p=0.0044)$. Even though the amount of histamine in the brain was not significantly different, the difference in ratios indicates that there are alterations in the metabolism of histamine relative to the wild type fly. In the eyes (Figure 4B), there was a main effect of genotype on the HA:CA ratio (one-way ANOVA, $p<0.0001$ ) and the $\mathrm{HA}: \mathrm{CA}$ ratio was significantly higher in ebony ${ }^{1}$ than Canton $\mathrm{S}$ or $\tan ^{3}$ eyes (Bonferroni post test, $p=0.0001$ and $p<0.0001$ respectively) and in Canton $S$ than $\tan ^{3}$ eyes $(p=0.0451)$. In the cuticle, there was a main effect of genotype on the HA:CA ratio (oneway ANOVA, $p=0.0297$ ) and HA:CA ratio was only significantly different between the ebony ${ }^{1}$ and $\tan ^{3}$, with ebony $y^{1}$ flies having a higher ratio than $\tan ^{3}$ flies $(p=0.0350)$. The consistent trend here is that the ratio of HA to CA is lowest in $\tan ^{3}$, due to deficits of the metabolism of histamine to carcinine and highest in ebony ${ }^{1}$ flies, due to deficits in the synthesis of carcinine from histamine. 
While mutations to Ebony and Tan were anticipated to alter the histamine content, our main finding is that the greatest effects these mutations are on the carcinine content and not on histamine content. Previous studies utilized whole heads for histamine and carcinine determination, while we analyzed more specific tissue types. ${ }^{15,11}$ For example, the whole head histamine content reported in Oregon $\mathrm{R}$ (another wild type strain of Drosophila) was $1980 \pm 150 \mathrm{pg} .{ }^{15}$ As the contribution of the eyes is larger than brains ( $270 \pm 80 \mathrm{pg}$ in the eyes of Canton S vs $44 \pm 15 \mathrm{pg}$ in the brain), previous whole head reports likely reflect eye and cuticle values more than they do brain values. The whole head contents of histamine in $\tan ^{3}$ and ebony ${ }^{1}$ were $200 \mathrm{pg} / \mathrm{head}$ and 900 pg/head respectively. ${ }^{16}$ Previous studies could not detect carcinine directly, but used feedings of $\left[{ }^{3} \mathrm{H}\right]$ histamine to produce $\left[{ }^{3} \mathrm{H}\right]$ carcinine, which could be measured. However, carcinine was only detected in $\tan ^{3}$ flies, which is consistent with our findings of elevated carcinine in $\tan ^{3}$ tissue types. Our method allows for direct comparison of histamine and carcinine and reveals that changes in metabolism affect carcinine more than histamine in the eyes, where visual processing is expected to take place.

The HA:CA ratios illustrate that there are differences in the relative amounts of these neurotransmitters that are dependent on genotype; ebony ${ }^{1}$ flies have a greater amount of histamine relative to carcinine than $\tan ^{3}$ flies in both their brains and eyes. While there are phenotypical changes associated with deficits in either of $\mathrm{N}$ - $\beta$-alanyl dopamine synthetase (ebony, which results in substantially darker cuticle than wild type) or $\beta$-alanyl-dopamine hydrolase (tan, which results in lighter cuticle than wild type), these changes do not appear to significantly change the neurotransmitter content of cuticle. The largest effects of Tan and Ebony on neurotransmitter content are in the brain and the eye, which agrees with immunohistochemical staining showing histamine reactivity in the eye and the optic lobes. ${ }^{6}$ As such, future work on histamine and carcinine in Drosophila should focus on the analysis of eyes and brains from other mutants. 


\subsubsection{Quantification of $\mathbf{N}-\beta$-alanyl-dopamine in $\tan ^{3}$ flies}

$\mathrm{N}$ - $\beta$-alanyl dopamine (BADA) is a metabolite of dopamine and is synthesized by $\mathrm{N}$ - $\beta$-alanyl dopamine synthetase (Ebony). This metabolite is typically found in the cuticle and eyes of insects, where it is involved in the pigmentation and sclerotization of these tissues. ${ }^{20,25,26}$ The primary role of $\mathrm{N}$ - $\beta$-alanyl dopamine in Drosophila appears to be in cuticle sclerotization, however there is evidence that Ebony is expressed in the head and

A.

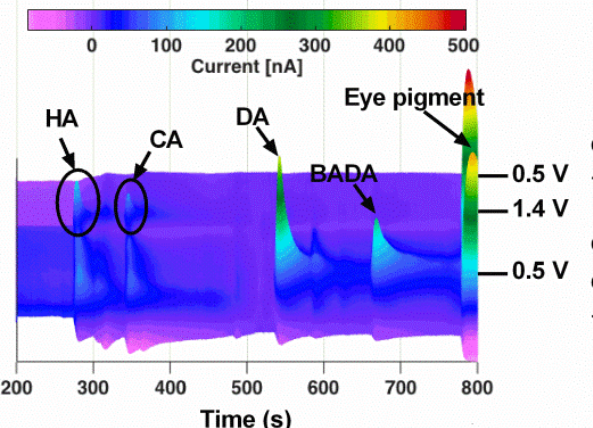

B.
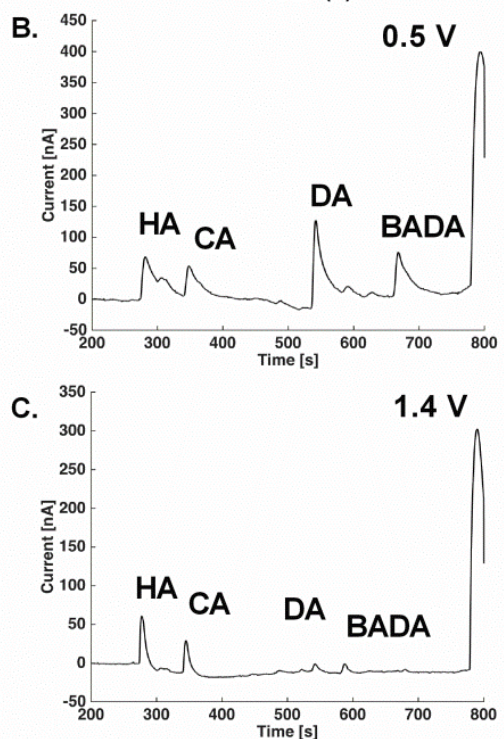

Figure 3-5: Separations of neurotransmitters in the eyes of a tan 3 fly. A. Heat map of the separation, with histamine, carcinine, dopamine and $\mathrm{N}$ - $\square$-alanyl dopamine circled. The last peak is an eye pigment only observed in eye samples. The bottom traces are electropherograms at different potentials. B. At $0.5 \mathrm{~V}, \mathrm{DA}$ and BADA are more visible. C. At $1.4 \mathrm{~V}, \mathrm{HA}$ and $\mathrm{CA}$ are more visible. Adapted (with permission) from Denno et al. 2016. brain of Drosohila. ${ }^{9}$ To the best of our knowledge, there is no evidence of $\mathrm{N}-\beta$ alanyl dopamine in mammals, and $\mathrm{N}-\beta$ alanyl dopamine is primarily of importance to insects.

$N$ - $\beta$-alanyl dopamine (BADA) was

not detected in most tissue types or strains of Drosophila tested, however it was detected consistently in $\tan ^{3}$ cuticle and eye samples (Figure 5). N- $\beta$-alanyl dopamine appears later in the separation than dopamine (Figure 5A, B), around 700 seconds (or $\sim 11.5 \mathrm{~min}$ ) and has a similar cyclic voltammogram to dopamine (Figure 1A), due to similar electrochemistry. $\mathrm{N}-\beta-$ alanyl dopamine is well separated from dopamine and other interferents in eye samples (Figure 5). 
The tissue content (in $\mathrm{pg} / \mathrm{mg}$ tissue) of $\mathrm{N}$ - $\beta$-alanyl dopamine of the cuticle and eyes for $\tan ^{3}$ were significantly different (Figure $6 \mathrm{~A}$ ) (unpaired t-test, $\mathrm{p}=0.0148$ ). The average content of $\mathrm{N}-\beta$-alanyl dopamine in $\tan ^{3}$ eyes was $56,000 \pm 16,000 \mathrm{pg} / \mathrm{mg}$ tissue and cuticle was $440 \pm 200 \mathrm{pg} / \mathrm{mg}$ tissue.

The ratio of the precursor to metabolite (DA:BADA) was calculated and no significant difference was found between the cuticle and the eyes (Fig. 6B) (unpaired ttest, $p=0.2193) . N-\beta$-alanyl dopamine was not detected in any other strain of fly, indicating that levels are below our limit of detection $(9 \pm 3 \mathrm{pg})$. Higher levels of $\mathrm{N}-\beta$ alanyl dopamine are present in $\tan ^{3}$ flies due to the absence of $\beta$-alanyl-dopamine hydrolase, which allows it to accumulate in tissue. While there is evidence of $\mathrm{N}$ - $\beta$-alanyl dopamine synthetase activity in the head of Drosophila, ${ }^{9}$ and we observed carcinine in

A.

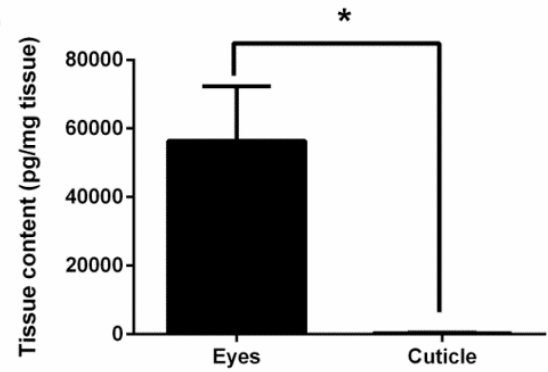

B.

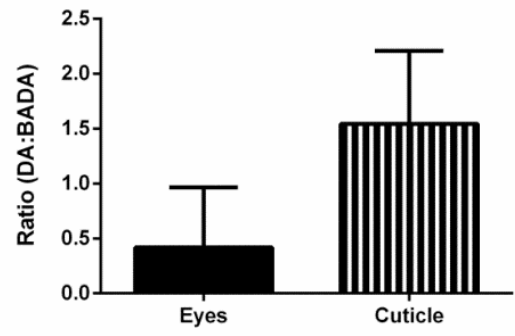

Figure 3-6: Tissue content of $N$ - $\beta$-alanyldopamine and ratios of dopamine to $N-\beta$ alanyl-dopamine in tan3. A. Content of $\mathrm{N}-\beta$ alanyl-dopamine in $\tan 3$ tissues: cuticle $(n=5)$ and eyes $(n=7)$. No N- $\beta$-alanyl-dopamine was detected in the brain of $\tan 3$ flies. The eyes contained significantly more $\mathrm{N}$ - $\beta$-alanyldopamine than the cuticle (Unpaired t-test, $\mathrm{p}=$ 0.0148). B. Comparisons of ratios of dopamine to $\beta$-alanyl-dopamine content (DA:BADA) in tan3 flies. There was no significant difference in the ratios from these tissues (t-test, $\mathrm{P}=0.0940$ ). * $\mathrm{p}<0.05$. Adapted (with permission) from Denno et al. 2016.

the brain, BADA levels may be lower because dopamine may not be available as a substrate in the brain or $\mathrm{N}$ - $\beta$-alanyl dopamine synthetase may be compartmentalized in different cell types than dopamine. The presence of $\mathrm{N}$ - $\beta$-alanyl dopamine in both the cuticle and the eye of $\tan ^{3}$ mutants agree well with our histamine and carcinine results, where the amount of metabolite in a tissue increases as $\mathrm{N}$ - $\beta$-alanyl dopamine hydrolase activity is abolished. Overall, $\mathrm{N}$ - $\beta$-alanyl dopamine content of wild type tissues appears to be extremely low, and as such may not be an important metabolite of dopamine, other 
than its role in normal pigmentation of cuticular tissues. Additionally, the extremely low level of $\mathrm{N}-\beta$-alanyl dopamine in the brain of $\tan ^{3}$ flies also indicates that $\mathrm{N}$ - $\beta$-alanyl dopamine is unlikely to be an important product of dopamine metabolism in brain tissue.

\subsubsection{Conclusions}

Here we report the first amount of histamine and carcinine in single brains as well as in eyes and cuticle from several strains of Drosophila melanogaster. Capillary electrophoresis with fast-scan cyclic voltammetry allows for highly sensitive detection of these analytes and resolves previous issues with carcinine detection without the need for sample pretreatment or derivitization. Furthermore, peak identities were confirmed by the use of cyclic voltammatery in addition to comparisons of migration times. Further application of CE-FSCV to study histamine and carcinine in a wider variety of Drosophila strains in the brain and eyes may help elucidate the impact of a variety of mutations on the visual system of Drosophila. Our method could also be used to determine histamine and carcinine in a variety of other invertebrates where histamine is suspected to be important for vision.

\subsection{Methods:}

\subsubsection{Chemicals}

Histamine and dopamine were purchased from Sigma Aldrich (St. Louis, MO). Carcinine, perchloric acid, and sodium phosphate monobasic monohydrate were purchased from Fisher Scientific (Pittsburgh, PA). N- $\beta$-alanyl-dopamine was obtained from NIMH Chemical Synthesis and Drug Supply Library (compound A-902). Perchloric acid was diluted to $5 \mathrm{mM}$ for fly tissue samples, and was diluted to $0.1 \mathrm{M}$ for the preparation of neurotransmitter stock solutions for calibrations. 


\subsubsection{Capillary Electrophoresis}

The small diameter fused silica capillary (10 $\mu$ m i.d., $151 \mu \mathrm{m}$ o.d., Polymicro Technologies, Phoenix, AZ) cut to $40-42 \mathrm{~cm}$ long. The detection end of the capillary had the polyimide coating removed from the first $\sim 2 \mathrm{~cm}$ by burning and was polished at a right angle on a polishing wheel (Sutter Instruments, Novoto, CA) . The separation capillary was filled with separation buffer prior to use and allowed to equilibrate for 15 minutes. Sample injection was electrokinetic: $+5 \mathrm{kV}$ was applied for $15 \mathrm{~s}$ at the injection end of the capillary through a platinum wire in the sample vial using a DC power supply (Spellman, Plainview, NY). The $+15 \mathrm{kV}$ separation voltage was applied at the injection end of the capillary through a platinum wire placed in a buffer reservoir. The detection end was grounded through stainless steel tubing attached to the detection cell, a Lucite block. ${ }^{17}$ The block was placed on a Stereomaster microscope (Fisher, Fair Lawn, NJ) to align the capillary and disk electrode, as previously reported. ${ }^{17} \mathrm{~A}$ cross flow buffer (detection buffer) flowed slowly $(0.5 \mathrm{~mL} / \mathrm{min})$ between the other arms to flush the area around the electrode.

\subsubsection{Buffer Composition}

Dissection buffer for Drosophila (modified PBS, pH 7.4) was made as follows: 131.25 mM NaCl, $3.0 \mathrm{mM} \mathrm{KCl}, 10 \mathrm{mM} \mathrm{NaH}_{2} \mathrm{PO}_{4}, 1.2 \mathrm{mM} \mathrm{MgCl}_{2}, 2.0 \mathrm{mM} \mathrm{Na}_{2} \mathrm{SO}_{4}, 1.2$ $\mathrm{mM} \mathrm{CalCl}$. After buffer was made and brought to the proper $\mathrm{pH}, 50 \mathrm{~mL}$ aliquots were made. $0.1 \mathrm{~g}$ of both trehalose and glucose were added to these aliquots to maintain tissue viability. Buffer with added sugars was discarded after use to reduce the possibility of bacterial contamination. 
Separation buffer was $200 \mathrm{mM}$ phosphate, $\mathrm{pH}$ 2.0, with $\mathrm{pH}$ adjusted using $\mathrm{HCl}$. The crossflow buffer was $100 \mathrm{mM}$ phosphate buffer, $\mathrm{pH}$ 7.0, with $\mathrm{pH}$ adjusted using $\mathrm{NaOH}$.

\subsubsection{Fast Scan Cyclic Voltammetry and Data Collection}

Detection was performed with fast-scan cyclic voltammetry in a two electrode configuration using a Dagan ChemClamp potentiostat (Dagan, Minneapolis, MN with a custom-modified headstage). Data acquisition software and hardware was as previously described. ${ }^{17}$ The electrode was scanned from $-0.4 \mathrm{~V}$ to $1.4 \mathrm{~V}$ and then back at $400 \mathrm{~V} / \mathrm{s}$ every $100 \mathrm{~ms}$. The CE-FSCV set up was kept inside of a Faraday cage in order to minimize the effects of external noise sources. Concentrations of neurotransmitters in fly tissue samples were determined by standard samples run before or after the fly sample. The oxidation peak current of the standard was used to determine the concentration per unit current $(n M / n A)$. This ratio was then used to convert the peak oxidation current $(n A)$ of the same analyte in the fly sample by multiplying by this ratio $(n M / n A)$.

\subsubsection{Heat map generation}

Heat maps were generated using a MATLAB program, written in house. The program takes color plot data generated by TarHeel CV or HDCV during the entire separation. These files are then concatenated and background subtraction is preformed within the program to generate the surface plots. The data is displayed as a surface plot rather than a false color intensity plot. This allows for easier visualization of peak heights and intensities in a way that combines the advantages of standard current vs. time traces we have previously used to show migration order with the advantages of false color plots traditionally used for display of CE-FSCV and FSCV data.

\subsubsection{Drosophila melanogaster sample preparation}


Drosophila melanogaster strains Canton S, ebony ${ }^{1}$, and $\tan ^{3}$ were maintained on a standard molasses yeast medium at $17^{\circ} \mathrm{C}$. Stocks of Canton S and ebony ${ }^{1}$ flies were obtained from Bloomington Drosophila Stock Center at Indiana University. $\operatorname{Tan}^{3}$ flies were generously provided by the Hirsh lab (University of Virginia). In ebony ${ }^{1}$ flies, there is a large scale deletion of the gene $N$ - $\beta$-alanyl dopamine synthetase. In $\tan ^{3}$ flies, a spontaneous mutation in $\mathrm{N}$ - $\beta$-alanyl dopamine hydrolase makes the gene non-functional. The mutants are commonly used for investigations into histamine and carcinine in the visual system. Canton S flies are used as a wild type strain of fly, and are used here to compare normal function of the visual system and normal histamine and carcinine cycling.

\subsubsection{Fly homogenate preparation}

Adult female flies were selected (3 days post eclosion), and dissected, removing the brain, both eyes, and the cuticle from the scutellum of the fly. These tissues were then placed in dissection buffer to maintain tissue viability and stored on ice in the dark. Sample vials were made as previously described ${ }^{17}$, and a new vial was used for each sample. Each sample vial was filled with $10 \mu \mathrm{L}$ of $0.5 \mathrm{mM}$ perchloric acid prior to adding tissue. Tissue samples (brain, eye, or cuticle) were removed from dissection buffer by pipetting, using a $10 \mu \mathrm{L}$ micropipette, and excess buffer was ejected into a waste container. The pipette tip was then stacked inside the sample vial. Each sample vial was loaded with a single brain, piece of cuticle, or the eyes of a single fly; no pooled samples with tissue from multiple flies were used. This allows for determination of neurotransmitter tissue content in a given tissue type obtained from individual flies. Both were then placed inside of a $2 \mathrm{~mL}$ centrifuge tube and were then centrifuged (Eppendorf, Brinkman Instruments, Westbury, NY) for 2 minutes at 9,800 rpm for $1 \mathrm{~min}$ at room temperature. After this, the pipette tip was checked to ensure that the tissue had moved 
into the sample vial. The tissue in the sample vial was homogenized using a thin, silver wire (28 gauge, o.d. $0.325 \mathrm{~mm}$ ). The sample vials were then transferred to capped $2 \mathrm{~mL}$ eppendorf tubes and were sonicated in a bath sonicator for 15 minutes. After sonication, the sample vials are inverted on top of Ultrafree centrifugal filter tubes (Millipore, Billerica, MA, USA) and were centrifuged for 4 minutes at 11,000 rpm. The filter was then removed and the filtrate was transferred to labeled $500 \mu \mathrm{L}$ Eppendorf tubes. Injections were performed from these tubes.

Fly tissue weights were obtained by weighing tissue from several flies and obtaining an average tissue weight for the brain $(0.035 \mathrm{mg} / \mathrm{brain})$, eyes $(0.022 \mathrm{mg} / \mathrm{two}$ eyes), and cuticle $(0.7 \mathrm{mg} /$ cuticle piece). Pooled weights were necessary as the balance was not sensitive enough to weigh single tissue samples. These weights were then used to determine the tissue content per $\mathrm{mg}$ of each tissue tested ( $\mathrm{pg} / \mathrm{mg}$ tissue).

\subsubsection{Statistics}

Error bars are standard error of the mean (SEM). Statistics were performed in GraphPad Prism 6 (La Jolla, CA).Outlier testing (Q-test, conservative) was performed on all data to remove definitive outliers. For comparisons of two groups, t-tests were used. For comparisons of three groups, one-way ANOVAs with Bonferonni post-test were performed and for comparisons with two variables, two-way ANOVAs with Bonferonni post tests were performed.

\subsection{Acknowledgements:}

This work was funded by the NIH R01MH085159 and a Camille Dreyfus TeacherScholar award to BJV. We also thank Jay Hirsh (University of Virginia, Dept. of Biology) for conversations with us during the planning and preparation of this manuscript as well as contributing a strain of flies used in this work. 


\subsection{References}

(1) Denno, M. E., Privman, E., Borman, R. P., Wolin, D. C., and Venton, B. J. (2016) Quantification of Histamine and Carcinine in Drosophila melanogaster Tissues. ACS Chem. Neurosci. 7, 407-414.

(2) Haas, H. L., Sergeeva, O. A., and Selbach, O. (2008) Histamine in the nervous system. Physiol. Rev. 88, 1183-241.

(3) Nitabach, M. N., and Taghert, P. H. (2008) Organization of the Drosophila circadian control circuit. Curr. Biol. 18, R84-93.

(4) Stuart, A. E. (1999) From Fruit Flies to Barnacles, Histamine Is the Neurotransmitter of Arthropod Photoreceptors. Neuron 22, 431-433.

(5) Monastirioti, M. (1999) Biogenic amine systems in the fruit fly Drosophila melanogaster. Microsc. Res. Tech. 45, 106-21.

(6) Melzig, J., Burg, M., Gruhn, M., Pak, W. L., and Buchner, E. (1998) Selective Histamine Uptake Rescues Photo- and Mechanoreceptor Function of Histidine Decarboxylase-Deficient Drosophila Mutant. J. Neurosci. 18, 7160-7166.

(7) Gavin, B. A., Arruda, S. E., and Dolph, P. J. (2007) The role of carcinine in signaling at the Drosophila photoreceptor synapse. PLoS Genet. 3, e206.

(8) Chaturvedi, R., Reddig, K., and Li, H.-S. (2014) Long-distance mechanism of neurotransmitter recycling mediated by glial network facilitates visual function in Drosophila. Proc. Natl. Acad. Sci. 111, 2812-2817.

(9) Pérez, M., Schachter, J., and Quesada-Allué, L. A. (2004) Constitutive activity of Nbeta-alanyl-catecholamine ligase in insect brain. Neurosci. Lett. 368, 186-91.

(10) Ziegler, A. B., Brüsselbach, F., and Hovemann, B. T. (2013) Activity and coexpression of Drosophila black with ebony in fly optic lobes reveals putative cooperative tasks in vision that evade electroretinographic detection. J. Comp. Neurol. $521,1207-24$.

(11) True, J. R., Yeh, S.-D., Hovemann, B. T., Kemme, T., Meinertzhagen, I. A., Edwards, T. N., Liou, S.-R., Han, Q., and Li, J. (2005) Drosophila tan encodes a novel hydrolase required in pigmentation and vision. PLoS Genet. 1, e63.

(12) Takahashi, A. (2013) Pigmentation and behavior: potential association through pleiotropic genes in Drosophila. Genes Genet. Syst. 88, 165-74.

(13) Hashemi, P., Dankoski, E. C., Wood, K. M., Ambrose, R. E., and Wightman, R. M. (2011) In vivo electrochemical evidence for simultaneous 5-HT and histamine release in the rat substantia nigra pars reticulata following medial forebrain bundle stimulation. $J$. Neurochem. 118, 749-59.

(14) Samaranayake, S., Abdalla, A., Robke, R., Wood, K. M., Zeqja, A., and Hashemi, P. (2015) In vivo histamine voltammetry in the mouse premammillary nucleus. Analyst 140, 
$3759-65$.

(15) Borycz, J., Vohra, M., Tokarczyk, G., and Meinertzhagen, I. A. (2000) The determination of histamine in the Drosophila head. J. Neurosci. Methods 101, 141-148.

(16) Borycz, J., Borycz, J. A., Loubani, M., and Meinertzhagen, I. A. (2002) tan and ebony Genes Regulate a Novel Pathway for Transmitter Metabolism at Fly Photoreceptor Terminals. J. Neurosci. 22, 10549-10557.

(17) Fang, H., Vickrey, T. L., and Venton, B. J. (2011) Analysis of Biogenic Amines in a Single Drosophila Larva Brain by Capillary Electrophoresis with Fast-Scan Cyclic Voltammetry Detection. Anal. Chem. 83, 2258-2264.

(18) Denno, M. E., Privman, E., and Venton, B. J. (2015) Analysis of neurotransmitter tissue content of Drosophila melanogaster in different life stages. ACS Chem. Neurosci. $6,117-23$.

(19) Jones, L. J., McCutcheon, J. E., Young, A. M. J., and Norton, W. H. J. (2015) Neurochemical measurements in the zebrafish brain. Front. Behav. Neurosci. 9, 246.

(20) Aust, S., Brüsselbach, F., Pütz, S., and Hovemann, B. T. (2010) Alternative Tasks of Drosophila Tan in Neurotransmitter Recycling Versus Cuticle Sclerotization Disclosed by Kinetic Properties. J. Biol. Chem. 285, 20740-20747.

(21) Chang, S.-Y., Jay, T., Muñoz, J., Kim, I., and Lee, K. H. (2012) Wireless fast-scan cyclic voltammetry measurement of histamine using WINCS--a proof-of-principle study. Analyst 137, 2158-65.

(22) Pihel, K., Hsieh, S., Jorgenson, J. W., and Wightman, R. M. (1995) Electrochemical detection of histamine and 5-hydroxytryptamine at isolated mast cells. Anal. Chem. 67, 4514-4521.

(23) Bullock, J. A., and Yuan, L.-C. (1991) Free solution capillary electrophoresis of basic proteins in uncoated fused silica capillary tubing. J. Microcolumn Sep. 3, 241-248.

(24) Dos Santos, G., Schroeder, A. J., Goodman, J. L., Strelets, V. B., Crosby, M. A., Thurmond, J., Emmert, D. B., and Gelbart, W. M. (2015) FlyBase: introduction of the Drosophila melanogaster Release 6 reference genome assembly and large-scale migration of genome annotations. Nucleic Acids Res. 43, D690-7.

(25) Wittkopp, P. J., Williams, B. L., Selegue, J. E., and Carroll, S. B. (2003) Drosophila pigmentation evolution: divergent genotypes underlying convergent phenotypes. Proc.

Natl. Acad. Sci. U. S. A. 100, 1808-13.

(26) Wright, T. R. (1987) The genetics of biogenic amine metabolism, sclerotization, and melanization in Drosophila melanogaster. Adv. Genet. 24, 127-222. 
\begin{tabular}{l|l} 
De n no & $\mathbf{8 4}$
\end{tabular} 
Chapter 4. Determination of Histamine and Carcinine Content in Drosophila melanogaster with visual deficits. 


\subsection{Abstract:}

Histamine and carcinine are involved in the visual transduction process of Drosophila melanogaster and are crucial for normal vision in Drosophila. Previously, I developed a capillary electrophoresis-fast scan cyclic voltammetry method for the detection and quantification of histamine and carcinine in tissues from Drosophila. Here, I quantitate histamine and carcinine content in several mutants with reduced vision:

eyeless $^{2}\left(\right.$ eye $\left.^{2}\right)$, eyes absent ${ }^{2}\left(\right.$ eya $\left.^{2}\right)$, sine oculis ${ }^{1}\left(s o^{1}\right)$, and glass ${ }^{3}\left(g \beta^{3}\right)$. White ${ }^{1118}$, which has been shown to have altered tissue content of neurotransmitters, was also analyzed. These strains were then compared to a wild type, Oregon R. Overall, the main finding is that histamine tissue content is lowered in the adult brain of sine oculis ${ }^{1}(1400 \pm 200$ $\mathrm{pg} / \mathrm{mg}$ tissue) and white ${ }^{1118}(1000 \pm 100 \mathrm{pg} / \mathrm{mg}$ tissue) compared to Oregon R (2600 \pm $200 \mathrm{pg} / \mathrm{mg}$ tissue). In the larval CNS, histamine content is lowered in eyeless ${ }^{2}(1000 \pm$ $100 \mathrm{pg} / \mathrm{mg}$ tissue) and eyes absent ${ }^{2}(1500 \pm 200 \mathrm{pg} / \mathrm{mg}$ tissue) compared to Oregon R $(2600 \pm 300 \mathrm{pg} / \mathrm{mg})$. However, carcinine content remains unchanged across all of the genotypes. Additionally, for those flies that have eyes, the tissue content in the eyes does not significantly change for histamine, and carcinine is only different in white ${ }^{1118}$. The decreased tissue content of histamine in these visual mutants contrasts with what we previously observed in metabolic mutants, where only carcinine content changed. These measurements demonstrate that alterations to the visual systems of Drosophila alter the tissue content of histamine, either through reduced synthesis of histamine or altered storage of histamine. 


\subsection{Introduction:}

The visual process in Drosophila occurs through the release and cycling of histamine, which is an active neurotransmitter, to carcinine, which is an inactive metabolite. ${ }^{1}$ This visual transduction process is common in invertebrates, and closely resembles the system of visual transduction used in mammals. ${ }^{2}$ The release and cycling of histamine to carcinine is necessary for normal visual and behavioral function in Drosophila: flies unable to metabolize histamine to carcinine (ebony) or carcinine back to histamine (tan), have been shown to have poor vision and inappropriately aggressive or passive behavior. ${ }^{3}$ This cycling is critical due to the slow rate of synthesis of histamine by histidine decarboxylase. However, in flies which cannot synthesize histamine at all $(H d c)$, normal eye development takes place but the eyes are not able to respond to visual stimulus. ${ }^{4}$

Many visual mutant strains have been made in Drosophila. These visual mutants are typically developmental mutants, where some aspect of development of the eye or a part of the eye has been disrupted or entirely lost. However, other parts of the visual system may remain intact. For example, in sine oculis ${ }^{1}\left(s o^{1}\right)$ flies, the compound eye is normally formed, but the simple eyes typically found on the top of the head (the ocelli) fail to develop in adult flies. ${ }^{5,6}$ In eyes absent $\left(e^{2} a^{2}\right)$ and eyeless (eye ${ }^{2}$ ) adult flies, while no compound eye develops, the ocelli are still present and have some function. ${ }^{7,8}$ In glass $^{3}\left(g l^{\beta}\right)$ flies, the eye fails to develop normal photoreceptors and eye lamina., 90 White ${ }^{1118}\left(w^{1118}\right)$ flies represent a different kind of visual deficit: white pigmentation (a mutation of the wild type red eye pigment) reduces the light screening ability of the compound eye, causing desensitization in bright light and abnormal optimotor responses, despite the eyes being present and normally sized. ${ }^{11}$ 
Previously, we have determined the tissue content of histamine and carcinine in a wild type, Canton $\mathrm{S}$, as well as two metabolic mutants, ebony ${ }^{1}$ and $\tan ^{3}$, using capillary electrophoresis coupled to fast scan cyclic voltammetry (CE-FSCV). ${ }^{12}$ Direct detection of both histamine and carcinine without sample pretreatment was possible, and limits of detection were low enough to determine tissue content of the brain, eyes, and cuticle from a single fly. This allowed accurate tracking of the metabolism of histamine and its distribution in a variety of tissues. Previous work using HPLC with electrochemical detection to analyze visual mutants has indicated that the tissue content of histamine in whole heads of a variety of visual mutants is greatly reduced compared to wild type Drosophila. ${ }^{1,13}$ Tissue content of histamine in whole heads of some visual mutants (so most notably) has been previously determined, but there is little information on the impact of loss of vision or loss of part of the visual apparatus on the tissue content of the Drosophila brain. ${ }^{1,13,14}$ Furthermore, while white ${ }^{1118}$ flies have been used as a comparison for other strains of flies or as a genetic background, there is increasing evidence that $w^{1118}$ has altered trafficking of neurotransmitters and reduced tissue content of neurotransmitters, possibly including histamine. ${ }^{11}$ As histamine immunoreactive neurons project to the eyes, ocelli, and other associated structures, we hypothesize that the brain tissue content of histamine may be reduced in mutants who have lost or reduced function in these tissues, and that white flies will show altered tissue distributions of histamine relative to wild type flies. ${ }^{15}$

In this work, we determined the tissue content of histamine and carcinine in individual larval and adult brains of the visual mutants eye $e^{2}, e y a^{2}, s o^{1}, g f^{3}$, and $w^{1118}$ and in the wild type strain, Oregon R. Overall, $s 0^{1}$ and $w^{1118}$ had lower amounts of histamine in the adult brain than Oregon R. In the larval CNS, eye $e^{2}$ and eya ${ }^{2}$ had lower amounts of histamine than Oregon R. Tissue content distribution of carcinine was altered in 
white ${ }^{1118}$ relative to all other strains of flies with eyes. In adult flies with eyes $\left(s o^{1}, g{ }^{\beta}\right.$, $w^{1118}$, and Oregon $\mathrm{R}$ ) there were no differences in histamine and carcinine content of the eyes. Carcinine content did not alter in any of the tested tissues. Thus, there are deficiencies in histamine in some, but not all flies with visual defects, which may be attributable to loss of visual information, alteration of neurons containing histamine and carcinine, or the disruption of trafficking of histamine and carcinine.

\subsection{Results and Discussion:}

\subsubsection{Drosophila melanogaster strains: Developmental Mutants and Eyesight}

\section{A. Oregon R}

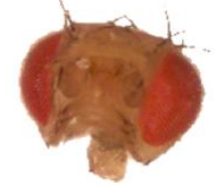

B. white ${ }^{1118}$

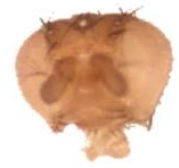

C. glass ${ }^{3}$

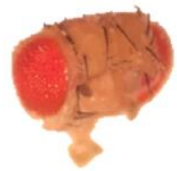

D. sine oculis ${ }^{1}$

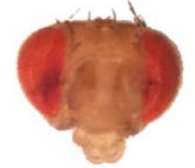

E. eyes absent ${ }^{2}$

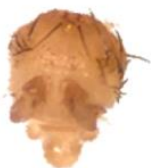

F. eyeless ${ }^{2}$

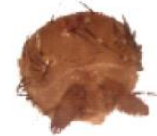

$1 \mathrm{~mm}$

Figure 4-1: Images of heads taken from adult $A$ ) Oregon R B) white ${ }^{1118}$ C) glass ${ }^{3}$ D) sine oculis ${ }^{1}$ E) eyes absent $^{2} F$ ) eyeless ${ }^{2}$ flies.
Several visual mutants

were selected to determine the

impact of altered vision on tissue

content of histamine and carcinine.

A variety of different mutants were

selected: pigmentation mutants,

mutants with improper

development of the surface of the

eye, mutants lacking ocelli, and

mutants that are completely

eyeless. The wild type, Oregon $\mathrm{R}$

(Figure $1 \mathrm{~A}$ ) has large, deep red

eyes and ocelli positioned on the

top of its head. The white ${ }^{1118}$

mutant has similarly sized unpigmented eyes (Figure $1 \mathrm{~B}$ ). In the glass ${ }^{3}$ mutant, the eyes are approximately the same size as Oregon $\mathrm{R}$, but appear rougher and lighter (Figure $1 \mathrm{C})$; the roughened surface of the eye is due to irregular development of "corneal 
nipples". ${ }^{10,16}$ The ocelli are smaller and sit closer to the cuticle. In sine oculis ${ }^{1}$ mutants, there is little difference in the size of the eyes, but the ocelli are greatly reduced or absent (Figure 1D). The most dramatic effect in appearance can be seen in eya ${ }^{2}$ and eye $e^{2}$ flies. In both eyes absent ${ }^{2}$ and eyeless ${ }^{2}$

(Figure $1 \mathrm{E}$ and $\mathrm{F}$ ) the ocelli are visible on the top of the head, but the eyes are entirely absent.

In addition to their roles in the development of the eye and associated structures, some of these genes encode for transcription factors (sine oculis and glass), transcription regulators (eyeless) or are involved with processes outside of the formation of the eye (Scheme 1). Another example of the regulatory nature of these genes

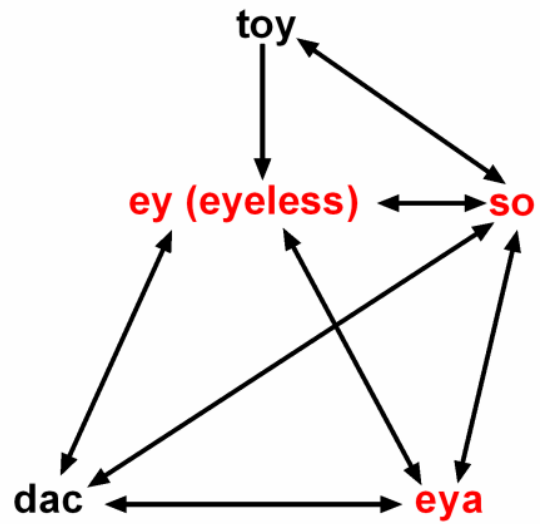

Scheme 4-1: A simplified schematic of the genetic regulatory network controlling eye formation in Drosophila. Genes highlighted in red represent the genes inactivated in mutants eye ${ }^{2}$, so $^{1}$, and eya ${ }^{2}$ respectively. Double headed arrows indicate reciprocal regulation. Of these genes, toy, eye, eya, and dac alone can induce eye development. Based on a scheme presented by Wagner, 2007. ${ }^{37}$

is eyes absent, which binds with sine oculis, regulating its activity (indicated by the double headed arrows in Scheme 1). As such, changes in the function of these genes may also have other effects that are not readily apparent from the phenotype, but may have negative impact on the normal development of the brain and other tissues where high expression levels of these genes are detected.

\subsubsection{Capillary electrophoresis for Histamine and Carcinine Detection}

Capillary electrophoresis coupled to fast scan cyclic voltammetry (CE-FSCV) was

used to determine the tissue content of brains and eyes from individual adults or larvae.

CE-FSCV is a fast, sensitive, and selective method for the determination of a wide variety of neurotransmitters in a variety of Drosophila tissues. ${ }^{12,17,18}$ Capillary 
electrophoresis is well suited for separations of these small tissue samples due to the small sample volumes required and the ability to integrate sample pre-concentration on capillary through field amplified sample stacking. ${ }^{19-21}$ FSCV provides sensitive detection of a wide variety of electroactive compounds, and provides identifying information on the compound detected from the characteristic cyclic voltammogram, which increases confidence in the identification of electroactive species in tissue samples. ${ }^{17,18}$

\section{A. Standards}

In order to sensitively detect histamine and

carcinine, we
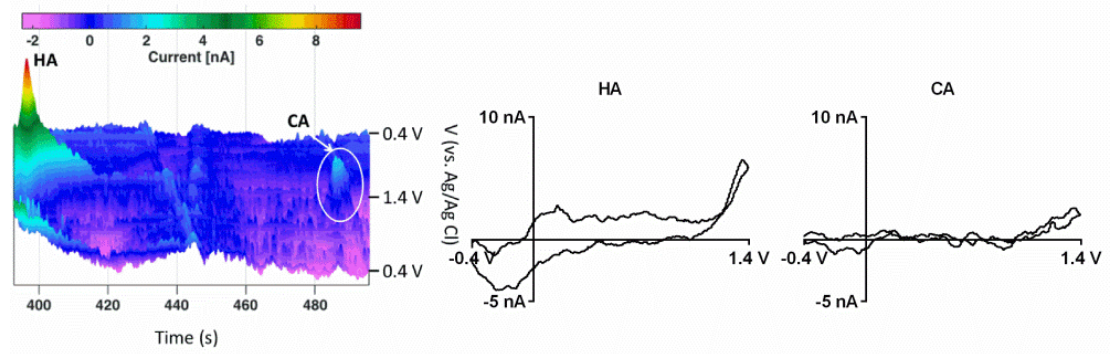

scanned from -0.4

$\mathrm{V}$ to $1.4 \mathrm{~V}$ at a rate B. $w^{1118}$ eyes

of $400 \mathrm{~V} / \mathrm{s}$ to better

visualize the

histamine and
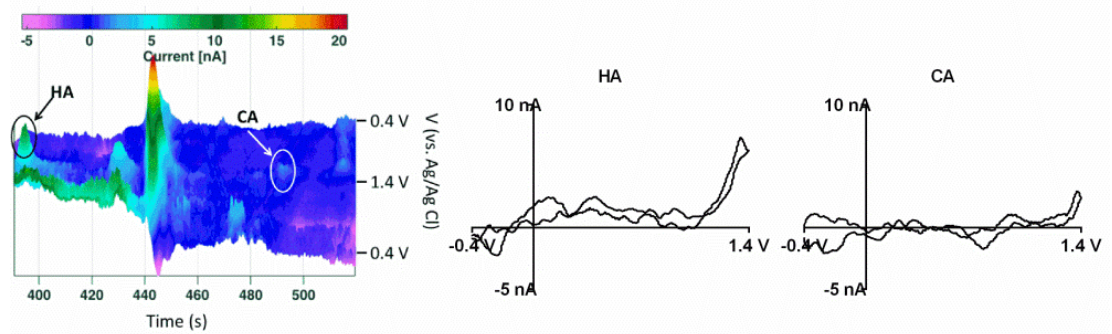

carcinine oxidation

peak. $^{22}$ The total

Figure 4-2: Example separation of A) $100 \mathrm{nM}$ standards and $B$ ) $w$ eye. In A) histamine (HA) can be seen on the color plot at $390 \mathrm{~s}$ and carcinine (CA) at $490 \mathrm{~s}$. In B) histamine can be seen on the color plot at 390 separation time $\mathrm{s}$ and carcinine can be seen at $490 \mathrm{~s}$. The cyclic voltammograms are shown on the right.

was only 8 minutes and histamine and carcinine were identified by matching migration times and comparing cyclic voltammograms to those obtained in standard samples.

Figure 2 shows data obtained from standards (Figure 2 A) and white ${ }^{1118}$ eyes (Figure 2 B) using CE-FSCV. The color plot (left) and the cyclic voltammograms (right) allow identification of compounds by characteristic cyclic voltammograms and by migration times. 


\subsubsection{Comparison of Tissue Content of Histamine and Carcinine in Different Tissues}

Using this CE-FSCV method, we

analyzed the tissue content of histamine

and carcinine in adult brains, adult eyes,

and larval central nervous systems

(CNS). Eye $e^{2}$ and eya ${ }^{2}$ lack eyes

completely, so eye tissue was not

A. Histamine

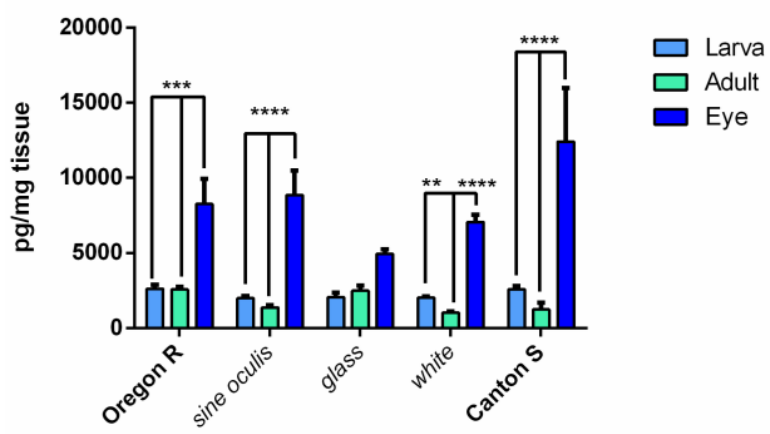

B. Carcinine

examined from them. Oregon $\mathrm{R}$ was

used as a wild type, due to its normal

eye and visual function. Additionally,

Oregon $\mathrm{R}$ is the genetic background on

which many of these mutants are made,

which makes it a more suitable control

than other wild types. Canton S, another

wild type we had previously analyzed,

was also included, to understand the

differences between these strains of

flies. The tissue content was corrected

for weight of tissue analyzed $(\mathrm{pg} / \mathrm{mg}$

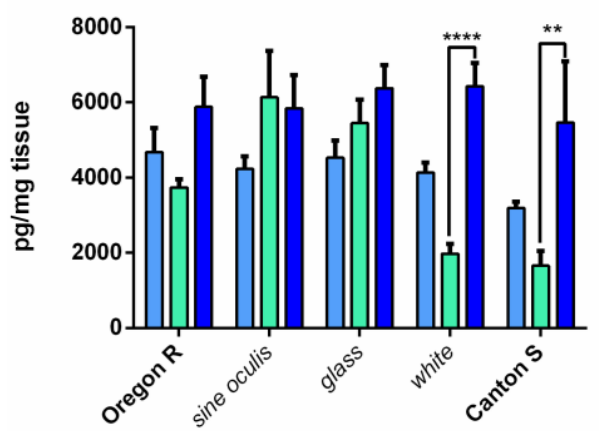

Figure 4-3: Comparison of tissue content of A) histamine $(n=5-12)$ and $B)$ carcinine $(n=5-12)$ in tissues from all strains with eyes. In A) the histamine content of the eyes is significantly higher in all strains (Bonferonni post test, $p=0.0042$ to $p$ $<0.0001$ ) than in the larval brain or the adult brain. This trend is absent in $g \beta^{3}$ tissues. In B) there is no significant difference in carcinine content of any of the tested tissue types, with the exception of $w$, where there is significantly more carcinine in the eyes than the adult brain (Bonferonni post test, $p<$ 0.0001 ), and Canton $S$, where the eyes have more carcinine than the adult brain (Bonferonni post test, $\mathrm{p}=0.0040) .{ }^{*}=\mathrm{P} \leq 0.05,{ }^{* *}=\mathrm{P} \leq 0.01,{ }^{* * *}=\mathrm{P} \leq$ $0.001,{ }^{* * * *}=\mathrm{P} \leq 0.0001$.

tissue) as the mass of the tissue did change with some of the mutations.

Figure 3 shows the histamine and carcinine levels in adult brains, larval CNS, and eyes in strains with adult eye formation. For histamine, there was no significant effect of genotype but there was a significant effect of tissue type (two-way ANOVA, $p<$ $0.0001)$, and a significant interaction between these variables $(p=0.0222)$ (Figure 3A). Comparisons between these tissues showed that histamine content was significantly 
higher in the eyes than in the brain for all strains tested (Bonferonni post test, $p=0.0009$ for Oregon $\mathrm{R}, \mathrm{p}<0.0001$ for all other strains) except glass ${ }^{3}$. Adult and larval brain histamine content do not significantly differ in any strain tested. The histamine content of the eyes was higher than the histamine content of the larval CNS in all tested strains (Bonferonni post test, $\mathrm{p}=0.0007$ for Oregon $\mathrm{R}, \mathrm{p}=0.0042$ for white ${ }^{1118}$, and $\mathrm{p}<0.0001$ for all others) except in glass ${ }^{3}$. For carcinine, there was a significant effect of genotype on tissue content (two way ANOVA, $\mathrm{p}=0.0015$ ) and a significant effect of tissue type (two way ANOVA, $p<0.0001$ ) (Figure 3B), and a significant interaction between these factors $(p=0.0319)$. Carcinine content was not significantly different in the eyes and the brain in any of the tested strains with the exception of both $w^{1118}$ and Canton S, where the eye contained significantly more carcinine than the brain (Bonferonni post test, $p<0.0001$ and $p=0.0040$ respectively). Carcinine content of the larval CNS was not significantly

different from the carcinine content of the eyes or adult brain.

In strains of flies without eyes $\left(e y e^{2}\right.$ and $\left.e y a^{2}\right)$, separate comparisons were made due to the lack of eye tissue. For histamine content, there was no significant effect of genotype, but there was a significant effect of tissue type on tissue content (two way ANOVA, $p=0.0003)$, and no significant interaction between tissue type and genotype (Figure 4A). There is significantly more histamine in eye $e^{2}$ adult brains than in the

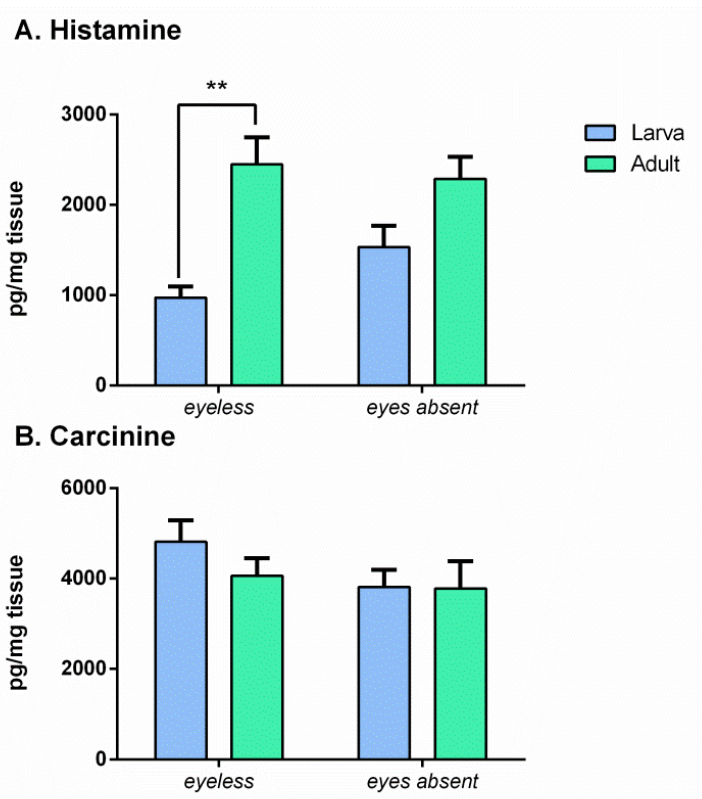

Figure 4-4: Comparison of tissue content of A) histamine $(n=11-12)$ and B) carcinine $(n=$ 11-12) in tissues from all strains without eyes. In A) the histamine content of eye adult brains is significantly higher than larval brains (Bonferonni post test $p=0.0019$ ). In $B$ ) there is no significant difference in carcinine content of any of the tested tissue types. ${ }^{* *}=\mathrm{P} \leq 0.01$ 
larval CNS (Bonferonni post test, $\mathrm{p}=0.0019)$. There was no difference in histamine content of eya ${ }^{2}$ larval CNS and adult brains. For carcinine tissue content, there was no significant effect of genotype or tissue type, and no significant interaction between these variables (two way ANOVA, $p>0.05$ ) (Figure 4B). Carcinine content did not significantly differ between the larval CNS and the adult brain in either strain of totally eyeless fly.

\subsubsection{Tissue Content of Histamine and Carcinine in Different Strains of Flies}

Another way to examine the impact of genotype on the tissue content of

histamine and carcinine is to look at individual tissue types. Figure 5 shows the tissue content of histamine and carcinine in the adult, larva, and the eyes. Overall, the main finding is that histamine tissue content in the adult brain $\left(s o^{1}\right.$ and $\left.w^{1118}\right)$ and the larval CNS $\left(e y e^{2}\right.$ and eya ${ }^{2}$ ) is lowered in some visual mutants. In addition, comparison with

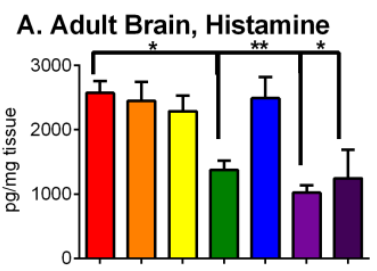

B. Adult Brain, Carcinine

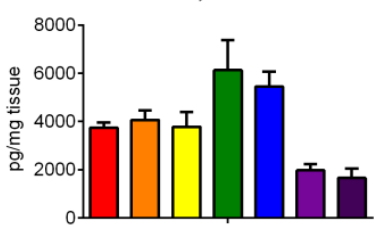

C. Larval CNS, Histamine

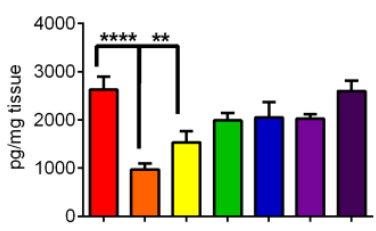

D. Larval CNS, Carcinine

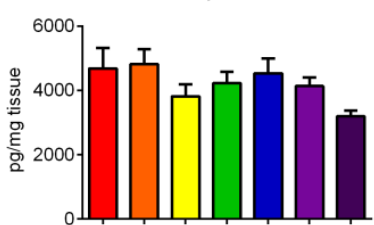

E. Eyes, Histamine

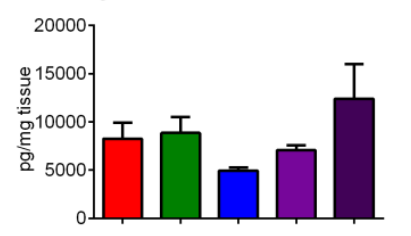

F. Eyes, Carcinine

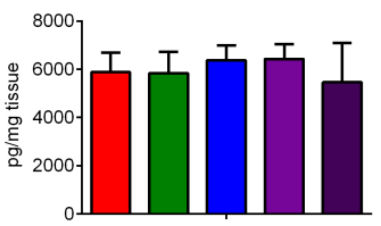

$\square$ Oregon R

$\square$ eyeless

$\square$ eyes absent

$\square$ sine oculis

glass

$\square$ white

$\square$ Canton S

Figure 4-5: Comparison of tissue content of histamine and carcinine in all of the different tissues tested.

A) histamine $(n=7-12)$ and $B)$ carcinine $(n=7-12)$ in adult brains taken from several different strains of Drosophila. In A) the histamine content of sine oculis $(p=0.0206$, One-way ANOVA with Bonferonni post test), white $(p=0.0015$, One-way ANOVA with Bonferonni post test), and Canton $S(p=0.0230$, One-way ANOVA with Bonferonni post test) brains is significantly lower than Oregon $R$. In $B$ ) there is no significant difference in carcinine content of any of the adult brains tested. C) histamine $(n=7-8)$ and $D)$ carcinine $(n=7-8)$ in the intact central nervous system of the 3rd instar larva. In C) the tissue content of histamine in Oregon $\mathrm{R}$ larval CNS is significantly higher than the eyeless $(p<0.0001$, One way ANOVA with Bonferonni post test) and the eyes absent $(p=0.0042$, One way ANOVA with Bonferonni post test) CNS tested. In D) there was no significant difference of carcinine tissue content of the CNS in any tested strain. F) histamine $(n=5-8)$ and F) carcinine $(n=5-8)$ in adult eyes. In both E) and F) there was no significant difference between the neurotransmitter tissue content of the tested strains. One-way ANOVA with Bonferonni post test, $p=0.2170, p=0.9141$ respectively). ${ }^{*}=P \leq 0.05$, ${ }^{* *}=\mathrm{P} \leq 0.01,{ }^{* * *}=\mathrm{P} \leq 0.001,{ }^{* * *}=\mathrm{P} \leq 0.0001$ 
another wild type fly, Canton S, shows reduced tissue content of histamine in the adult brain, but is otherwise statistically similar to Oregon R. In contrast to histamine, carcinine content remains unchanged across all of the genotypes. In those flies with eyes, the tissue content of both histamine and carcinine does not significantly change from the wild type.

In the adult brain, histamine content varied with genotype (one way ANOVA, $p$ $<0.0001$ ) (Figure 5A). Oregon $\mathrm{R}$ has significantly more histamine than $s 0^{1}$ (Bonferonni post test, $\mathrm{p}=0.0206), w^{1118}$ (Bonferonni post test, $\left.\mathrm{p}=0.0015\right)$, and Canton $\mathrm{S}$ (Bonferonni post test, $p=0.0230$ ) and there is no significant difference in the histamine content of other adult brains. Carcinine content in adult brains also differed significantly with genotype (one way ANOVA, $\mathrm{p}<0.0001$ ). However, carcinine content does not significantly differ from Oregon $R$ tissue content in any of the strains (Figure 5B). In the larval brain (Figure 5C), histamine significantly varied with genotype (one way ANOVA, p $<0.0001$ ) and Oregon R had significantly more histamine than either eye ${ }^{2}$ (Bonferonni post test, $p<0.0001$ ) or eya ${ }^{2}$ (Bonferonni post test, $p=0.0041$ ). Larval carcinine content did not significantly differ with genotype (one way ANOVA) and carcinine remained constant across the genotypes in larvae, similar to adult brains (Figure 5D). In the adult eye (Figure 5E), the tissue content of histamine (one way ANOVA, $p=0.1688$ ) or carcinine (one way ANOVA, $\mathrm{p}=0.9560$ ) did not vary significantly across tested strains. Again, similar to the other tissues tested, carcinine content does not significantly differ among the genotypes (Figure 5F). 


\subsection{Discussion}

The tissue content for each strain and each tissue type can be seen in Table 1.

Comparisons between the wild type

Canton $\mathrm{S}$ and Oregon $\mathrm{R}$ show that there are some differences in tissue content of histamine in the brain of different wild type strains. Other small differences in tissue distribution between the two strains can be seen as well, and differences in the body color of different wild type strains have been documented. ${ }^{23,24}$ As such, the expression of pigmentation genes which affect the synthesis of histamine may be between altered between these wild type strains, showing the importance of picking the correct wild type for comparisons.

In contrast to our previous work with metabolic mutants, we see that the effect of visual mutants is on histamine content, and not on carcinine content. ${ }^{12}$ However, we did expect differences in histamine here based on some of the existing literature. For sine oculis ${ }^{1}$ adult brains, our result agrees well with what was previously observed by Borycz and co-workers in whole heads: $s 0^{1}$ brain tissue content of histamine is lower than in wild type. ${ }^{1}$ We believe that this is due in part to reduced histaminergic inputs via loss of ocellus function. Additionally, this difference in histamine tissue content may also be due to the important developmental role of the sine oculis.
Table 4-2: Tissue content for all tissues tested in all strains of flies.

\begin{tabular}{|lcl|}
\hline & Oregon R & \\
Tissue type & Histamine & Carcinine \\
Adult Brain $(n=7)$ & $2600 \pm 200$ & $3700 \pm 200$ \\
Eyes $(n=7)$ & $8300 \pm 1700$ & $5900 \pm 800$ \\
Larval CNS $(n=8)$ & $2600 \pm 300$ & $4700 \pm 600$ \\
& Canton S & \\
Tissue type & Histamine & Carcinine \\
Adult brain $(n=7)$ & $1200 \pm 400$ & $2000 \pm 400$ \\
Eyes ( $n=7)$ & $12000 \pm 4000$ & $5500 \pm 2000$ \\
Larval CNS $(n=7)$ & $2600 \pm 200$ & $3200 \pm 200$ \\
& White & \\
Tissue type & Histamine & Carcinine \\
Adult Brain $(n=11)$ & $1000 \pm 100$ & $2000 \pm 300$ \\
Eyes ( $n=8)$ & $7000 \pm 500$ & $6400 \pm 600$ \\
Larval CNS $(n=6)$ & $2000 \pm 100$ & $4100 \pm 300$ \\
& Glass & \\
Tissue type & Histamine & Carcinine \\
Adult Brain $(n=11)$ & $2500 \pm 300$ & $5500 \pm 600$ \\
Eyes ( $n=5)$ & $4900 \pm 300$ & $6400 \pm 600$ \\
Larval CNS $(n=7)$ & $2100 \pm 300$ & $4500 \pm 500$ \\
& Sine oculis & \\
Tissue type & Histamine & Carcinine \\
Adult Brain $(n=12)$ & $1400 \pm 200$ & $6100 \pm 1300$ \\
Eyes ( $n=7)$ & $8900 \pm 1600$ & $5900 \pm 900$ \\
Larval CNS $(n=7)$ & $2000 \pm 200$ & $4200 \pm 400$ \\
& Eyeless & \\
Tissue type & Histamine & Carcinine \\
Adult Brain $(n=12)$ & $2500 \pm 300$ & $4100 \pm 400$ \\
Larval CNS $(n=6)$ & $1000 \pm 100$ & $4800 \pm 500$ \\
Tissue type & Eyes absent & \\
Adult Brain $(n=11)$ & $2300 \pm 300$ & $3800 \pm 600$ \\
Larval CNS $(n=8)$ & $1500 \pm 200$ & $3800 \pm 400$ \\
\hline
\end{tabular}

All tissue contents given in $\mathrm{pg} / \mathrm{mg}$ tissue. 
Sine oculis encodes a transcription factor, and expression of sine oculis in the developing brain may be necessary for normal development of the brain. ${ }^{15,25,26}$ As such, the change in histamine content of the adult brain may be due to a combination of these factors.

Understanding the reduced histamine content of adult brains in $w^{1118}$ is similarly complicated. The differences in tissue distribution of carcinine in addition to the reduced adult whole head content of histamine suggest that there is either reduced availability of both the neurotransmitter and its metabolite or a reduced ability to store histamine and carcinine. Previous work on $w^{1118}$ indicated that tissue content of a variety of neurotransmitters was lowered relative to wild type, and that white may encode a transporter responsible for trafficking of multiple neurotransmitters, including histamine. ${ }^{11}$ As such, our results confirm that there is some abnormality in tissue localization and distribution relative to the wild type fly.

In larvae, differences in the CNS content of histamine of eye ${ }^{2}$ and eya ${ }^{2}$ relative to the wild type may be due to alteration of the development of the Bolwig organ, which is the larva's visual system. ${ }^{27}$ This organ changes during pupation and is involved in the formation of non-compound eye structure development. ${ }^{5,8,28}$ In addition to the role of eye and eya in the development of the eye, they are also involved in the regulation of the expression of other genes in their function as a transcription factor and ability to bind to other transcription factors, respectively. As the pathway that eye and eya participate in is very complicated and in many cases relies on reciprocal activation to cause proper eye formation (Scheme 1), there is a possibility that in the larva there is already an altered regulation of other genes leading to reduced development of histaminergic neurons or inputs to the larval CNS. Outside of the Bolwig organ and visual development, there is evidence that eye and eya are expressed in other areas of the developing brain, which 
may also account for altered tissue content of histamine. ${ }^{29}$ In aggregate, these factors may cause different development of the larva CNS that evades visual inspection, but leads to differences in tissue content of histamine.

In addition to the difference in tissue content of histamine between wild type and eyeless larval CNS, eyeless larva and adults showed altered tissue content distribution relative to other strains tested. In eyeless larva, we observed significantly less histamine than in adult eyeless brains. We believe that this difference is due to the importance of expression of eyeless during the development of the visual system: eyeless guides development of the embryonic tissues that will develop into the eye (the eye primordia) and does so very early during development. ${ }^{27}$ Furthermore, eyeless expression appears to be necessary in the development of the Bolwig organ (the visual system of the larva). ${ }^{27}$ It is possible that improper development of the Bolwig organ may lead to reduced CNS histamine content. Visual inspection of eyeless larval CNS shows them to be similarly sized to wild type, but the development of the adult brain is clearly abnormal. This is likely due to the role of eyeless in preventing cell death and in the normal development of the brain. ${ }^{30,31}$ In this case, correcting for tissue weight can obscure the difference between the larva and the adult in this strain: the adult eyeless ${ }^{2}$ brain weighs $0.016 \mathrm{mg}$ whereas the larva weighs more than twice as much $(0.034 \mathrm{mg})$.

There are also factors that do not significantly change across the different strains and tissues tested. In flies with eyes, the tissue content of both histamine and carcinine do not significantly differ across strains. This similarity is likely due to the preservation of structures containing histamine and carcinine in the eyes. Even though some of the visual mutants have incorrect organization of the eye, such as incorrect projection of retinal axons in glass ${ }^{3}$, they still are present. The glia, which are responsible for metabolizing carcinine back to histamine, are also present. ${ }^{32}$ In other mutants we tested 
with eyes, these structures should be intact and not altered. Furthermore, changes to the size of the eyes are minimal in our tested strains with eyes, even in the $g^{\beta}$ flies.

In comparisons between strains of flies, carcinine was not significantly different in any tissue from Oregon R. Maintenance of the structures of the eye responsible for trafficking of histamine account for this in the eyes. Carcinine tissue content is controlled by the metabolism of histamine. ${ }^{33}$ As such, the content of carcinine may be controlled by the capacity of the tissue to use histamine in the visual transduction process or in the rate of metabolism back to histamine. Additionally, there are carcinine transporters that are present in photoreceptors that control the clearance of carcinine. ${ }^{34,35}$ Having these systems and structures intact and present in these tissues may allow for maintenance of carcinine content absent any loss of clearance ability or loss of ability to metabolize carcinine into histamine. Another aspect of carcinine tissue content that bears further investigation is the tissue content ratio (Histamine to Carcinine) in different tissues. As the rate of histamine synthesis through histidine decarboxylase is slow, the cycling of histamine to carcinine is likely to be the primary source of histamine. Therefore, storage as carcinine, the inactive form, may be preferred in visual mutants or in instances where histamine is not actively needed.

\subsection{Conclusions}

Histamine content is altered in some, but not all visual mutants. In the adult, $s 0^{1}$ and $w^{1118}$ brains have significantly reduced histamine content, which confirms previous work indicating that there was significantly less histamine in the whole head. ${ }^{1,11,36}$ In the larval CNS, both eye $e^{2}$ and eya ${ }^{2}$ have lower histamine tissue content than Oregon $\mathrm{R}$. These alterations are likely due to a combination of factors, such as the regulatory roles for the protein in the development of visual structures, the development of the brain or reduced or altered histaminergic inputs. In all tissue types, carcinine tissue content 
remains statistically similar; however, tissue distribution of carcinine is altered in $w^{1118}$ indicating that white does participate in the trafficking and localization of histamine as previously suggested. ${ }^{11}$ Overall, more targeted analysis of the visual system through the use of RNAi would assist in further understanding the link between vision and brain histamine and carcinine content.

\subsection{Methods}

\subsubsection{Chemicals}

Histamine and carcinine were purchased from Sigma-Aldrich (St. Louis, MO). Perchloric acid, sodium phosphate monobasic monohydrate were purchased from Fisher Scientific (Pittsburgh, PA). Perchloric acid was diluted to $0.1 \mathrm{M}$ for the preparation of neurotransmitter stock solutions, and was dilute to $0.5 \mathrm{mM}$ for use in fly tissue samples.

\subsubsection{Capillary Electrophoresis}

A 10 um inner diameter fused silica capillary (10 um i.d., 151 um o.d., Polymicro Technologies, Phoenix, AZ) was cut to $40-42 \mathrm{~cm}$ in length. The polyimide coating on the detection end of the capillary was removed by burning the last $2 \mathrm{~cm}$ of capillary. The separation capillary was filled with separation buffer prior to first use, and was allowed to equilibrate for 20 minutes. Injection of sample on to the capillary was electrokinetic: $+5 \mathrm{kV}$ was applied for 15 s at the injection end of the capillary using a silver wire in the sample vial. This wire was connected to a dc high voltage power supply (Spellman, Plainview, NY). After sample injection, the capillary and wire were moved back to the separation buffer containing vial. The $+15 \mathrm{kV}$ separation voltage was then applied at the injection end of the capillary through the silver wire in the buffer reservoir. Grounding at the detection end of the capillary was achieved through grounding a stainless steel tube that was attached to the detection cell. The lucite detection cell was placed under a Stereomaster microscope (Fisher, Fair Lawn, NJ) to allow accurate alignment of the 
detector with the disk electrode. ${ }^{12,17,18}$ A cross-flow buffer (detection cell buffer) was flowed slowly through the detection cell $(\sim 0.5 \mathrm{~mL} / \mathrm{min})$ to flush the area around the electrode and prevent buildup of analyte in the cell.

\subsubsection{Buffer Composition for CE}

The dissection buffer for Drosophila tissues (modified PBS, pH 7.4) was made as follows: $131.25 \mathrm{mM} \mathrm{NaCl}, 3.0 \mathrm{mM} \mathrm{KCl}, 10 \mathrm{mM} \mathrm{NaH}_{2} \mathrm{PO}_{4}, 1.2 \mathrm{mM} \mathrm{MgCl}_{2}, 2.0 \mathrm{mM}$ $\mathrm{Na}_{2} \mathrm{SO}_{4}, 1.2 \mathrm{mM} \mathrm{CalCl}_{2}$. After the buffer was brought up to the proper $\mathrm{pH}$, it was aliquoted into $50 \mathrm{~mL}$ portions. In each $50 \mathrm{~mL}$ aliquot, $0.1 \mathrm{~g}$ of both glucose and trehalose were added. These sugars help maintain tissue viability during dissection. After use, buffer was discarded to prevent contamination of tissue with bacteria.

The separation buffer was $200 \mathrm{mM}$ phosphate, at $\mathrm{pH}$ 2.0, adjusted down using $\mathrm{HCl}$. The crossflow buffer was $100 \mathrm{mM}$ phosphate buffer, $\mathrm{pH}$ 7.0, adjusted using $\mathrm{NaOH}$.

\subsubsection{Fast-Scan Cyclic Voltammetry and Data Collection}

Detection was performed with fast-scan cyclic voltammetry in a two electrode configuration using a Dagan ChemClamp potentiostat (Dagan, Minneapolis, MN, with a customized headstage). Data acquisition software and hardware were as previously described. ${ }^{17,18}$ For sensitive detection of histamine and carcinine, the electrode was scanned from $-0.4 \mathrm{~V}$ to $1.4 \mathrm{~V}$ and then back at a rate of $400 \mathrm{~V} / \mathrm{s}$ every $100 \mathrm{~ms}$. The CEFASCV set up was kept inside a large Faraday cage to reduce the effect of external sources of electrical noise. Concentrations of neurotransmitters in fly tissue samples was determined by standard samples run before or after the tissue sample run. The peak oxidation current of the standard $(\mathrm{nA})$ was used to determine the concentration per unit current $(\mathrm{nM} / \mathrm{nA})$. The ratio was then used to convert the peak oxidation current of the analyte in the tissue sample $(n A)$ to a concentration by multiplying by the $n M / n A$ value determined from the standard. This generates a nM value for the unknown. 


\subsubsection{Drosophila melanogaster Tissue Sample Preparation}

Drosophila melanogaster strains Oregon R (wild type), eye ${ }^{2}$, eya ${ }^{2}, s o 1, g \beta^{3}$, and $w^{1118}$ were maintained on a standard molasses yeast medium at $17^{\circ} \mathrm{C}$. All stocks with the exception of $e y e^{2}$ were obtained from Bloomington Drosophila Stock Center at Indiana University. Eye $e^{2}$ flies were generously provided by the Hirsh lab (University of Virginia).

\subsubsection{Tissue Homogenate Preparation}

Adult females were selected (3 days post eclosion) and were dissected to remove either the eyes or the brain of the adult fly. Larval CNS were dissected from wandering $3^{\text {rd }}$ instar larva. These tissues were conducted in dissection buffer and dissected tissues were kept in dissection buffer to maintain tissue viability. Sample transfer was performed as previously documented. ${ }^{12,17}$ Briefly: tissue samples were homogenized, sonicated in a bath sonicator, and were then filtered using a centrifugal filtration unit. This filtrate was then removed and stored in $.5 \mathrm{~mL}$ Eppendort tubes. These tubes were used for electrokinetic injection of sample. Sample vials were used for single brains, or a pair of eyes from a single fly. No pooled samples were used.

Tissue weights of brains and central nervous systems were obtained by weighing tissue from several flies and obtaining an average weight for the eyeless brain $(0.016 \mathrm{mg})$, eyes absent brain $(0.025 \mathrm{mg})$, and the wild type brain $(0.035 \mathrm{mg})$. The eyes $(0.022$ $\mathrm{mg} / \mathrm{two}$ eyes) and the weight of larval CNS $(0.034 \mathrm{mg})$ were also determined. These weights were then used to determine the tissue content per milligram of each tissue tested (pg/mg tissue), which allowed unbiased comparison between the different tissue types.

\subsubsection{Statistics}

Error bars are standard error of the mean (SEM). Statistics were performed in GraphPad Prism 6 (La Jolla, CA). All data was tested for outliers prior to any other data 
analysis, and outliers were removed (Q-test, conservative). For comparisons of three groups or more, one way ANOVA with Bonferonni post test was performed and for comparisons where there was more than one variable, two way ANOVA with Bonferonni post test was performed

\subsubsection{References.}

(1) Borycz, J., Vohra, M., Tokarczyk, G., and Meinertzhagen, I. A. (2000) The determination of histamine in the Drosophila head. J. Neurosci. Methods 101, 141-148.

(2) Stuart, A. E. (1999) From Fruit Flies to Barnacles, Histamine Is the Neurotransmitter of Arthropod Photoreceptors. Neuron 22, 431-433.

(3) Takahashi, A. (2013) Pigmentation and behavior: potential association through pleiotropic genes in Drosophila. Genes Genet. Syst. 88, 165-74.

(4) Melzig, J., Burg, M., Gruhn, M., Pak, W. L., and Buchner, E. (1998) Selective Histamine Uptake Rescues Photo- and Mechanoreceptor Function of Histidine Decarboxylase-Deficient Drosophila Mutant. J. Neurosci. 18, 7160-7166.

(5) Helfrich-Förster, C., Edwards, T., Yasuyama, K., Wisotzki, B., Schneuwly, S., Stanewsky, R., Meinertzhagen, I. A., and Hofbauer, A. (2002) The extraretinal eyelet of Drosophila: development, ultrastructure, and putative circadian function. J. Neurosci. 22, 9255-66.

(6) Buschbeck, E. K., and Friedrich, M. (2008) Evolution of Insect Eyes: Tales of Ancient Heritage, Deconstruction, Reconstruction, Remodeling, and Recycling. Evol. Educ. Outreach 1, 448-462.

(7) Punzo, C., Kurata, S., and Gehring, W. J. (2001) The eyeless homeodomain is dispensable for eye development in Drosophila. Genes Dev. 15, 1716-23.

(8) Bonini, N. M., Leiserson, W. M., and Benzer, S. (1993) The eyes absent gene: genetic control of cell survival and differentiation in the developing Drosophila eye. Cell 72, 379-95.

(9) Moses, K., and Rubin, G. M. (1991) Glass encodes a site-specific DNA-binding protein that is regulated in response to positional signals in the developing Drosophila eye. Genes Dev. 5, 583-93.

(10) Stark, W. S., and Carlson, S. D. (1991) Comparison of the surfaces of "glnone"s' ocelli and compound eyes with those of several glass alleles. Drosoph. Inf. Serv. 70, 217-219.

(11) Borycz, J., Borycz, J. A., Kubów, A., Lloyd, V., and Meinertzhagen, I. A. (2008) Drosophila $A B C$ transporter mutants white, brown and scarlet have altered contents and distribution of biogenic amines in the brain. J. Exp. Biol. 211, 3454-66.

(12) Denno, M. E., Privman, E., Borman, R. P., Wolin, D. C., and Venton, B. J. (2016) Quantification of Histamine and Carcinine in Drosophila melanogaster Tissues. ACS 
Chem. Neurosci.

(13) Sarthy, P. V. (1991) Histamine: A Neurotransmitter Candidate for Drosophila Photoreceptors. J. Neurochem. 57, 1757-1768.

(14) Borycz, J., Borycz, J. A., Loubani, M., and Meinertzhagen, I. A. (2002) tan and ebony Genes Regulate a Novel Pathway for Transmitter Metabolism at Fly Photoreceptor Terminals. J. Neurosci. 22, 10549-10557.

(15) Rieger, D., Stanewsky, R., and Helfrich-Förster, C. (2003) Cryptochrome, Compound Eyes, Hofbauer-Buchner Eyelets, and Ocelli Play Different Roles in the Entrainment and Masking Pathway of the Locomotor Activity Rhythm in the Fruit Fly Drosophila Melanogaster. J. Biol. Rhythms 18, 377-391.

(16) Stark, W. S., Sapp, R., and Carlson, S. D. (2009) Ultrastructure of the Ocellar Visual System in Normal and Mutant Drosophila Melanogaster. J. Neurogenet. 5, 127-153.

(17) Denno, M. E., Privman, E., and Venton, B. J. (2015) Analysis of neurotransmitter tissue content of Drosophila melanogaster in different life stages. ACS Chem. Neurosci. $6,117-23$.

(18) Fang, H., Vickrey, T. L., and Venton, B. J. (2011) Analysis of Biogenic Amines in a Single Drosophila Larva Brain by Capillary Electrophoresis with Fast-Scan Cyclic Voltammetry Detection. Anal. Chem. 83, 2258-2264.

(19) Bergquist, J., Ściubisz, A., Kaczor, A., and Silberring, J. (2002) Catecholamines and methods for their identification and quantitation in biological tissues and fluids. $J$. Neurosci. Methods 113, 1-13.

(20) and, C.-X. Z., and Thormann*\%, W. (1996) Head-Column Field-Amplified Sample Stacking in Binary System Capillary Electrophoresis: A Robust Approach Providing over 1000-Fold Sensitivity Enhancement.

(21) Weng, Q., Xu, G., Yuan, K., and Tang, P. Determination of monoamines in urine by capillary electrophoresis with field-amplified sample stacking and amperometric detection. J. Chromatogr. B.

(22) Heien, M. L. A. V, Phillips, P. E. M., Stuber, G. D., Seipel, A. T., and Wightman, R. M. (2003) Overoxidation of carbon-fiber microelectrodes enhances dopamine adsorption and increases sensitivity. Analyst. The Royal Society of Chemistry.

(23) Wittkopp, P. J., Williams, B. L., Selegue, J. E., and Carroll, S. B. (2003) Drosophila pigmentation evolution: divergent genotypes underlying convergent phenotypes. Proc. Natl. Acad. Sci. U. S. A. 100, 1808-13.

(24) Takahashi, A., Takahashi, K., Ueda, R., and Takano-Shimizu, T. (2007) Natural variation of ebony gene controlling thoracic pigmentation in Drosophila melanogaster. Genetics 177, 1233-7.

(25) Chang, T., Younossi-Hartenstein, A., and Hartenstein, V. (2003) Development of neural lineages derived from the sine oculis positive eye field of Drosophila. Arthropod Struct. Dev. 32, 303-17.

(26) Serikaku, M. A., and O'Tousa, J. E. (1994) sine oculis is a homeobox gene required 
for Drosophila visual system development. Genetics 138, 1137-50.

(27) Sheng, G., Thouvenot, E., Schmucker, D., Wilson, D. S., and Desplan, C. (1997) Direct regulation of rhodopsin 1 by Pax-6/eyeless in Drosophila: evidence for a conserved function in photoreceptors. Genes Dev. 11, 1122-1131.

(28) Suzuki, T., and Saigo, K. (2000) Transcriptional regulation of atonal required for Drosophila larval eye development by concerted action of eyes absent, sine oculis and hedgehog signaling independent of fused kinase and cubitus interruptus. Development 127, 1531-1540.

(29) Kurusu, M., Nagao, T., Walldorf, U., Flister, S., Gehring, W. J., and FurukuboTokunaga, K. (2000) Genetic control of development of the mushroom bodies, the associative learning centers in the Drosophila brain, by the eyeless, twin of eyeless, and Dachshund genes. Proc. Natl. Acad. Sci. U. S. A. 97, 2140-4.

(30) Callaerts, P., Leng, S., Clements, J., Benassayag, C., Cribbs, D., Kang, Y. Y., Walldorf, U., Fischbach, K. F., and Strauss, R. (2001) Drosophila Pax-6/eyeless is essential for normal adult brain structure and function. J. Neurobiol. 46, 73-88.

(31) Benassayag, C. (2003) Evidence for a direct functional antagonism of the selector genes proboscipedia and eyeless in Drosophila head development. Development 130, 575-586.

(32) Chaturvedi, R., Reddig, K., and Li, H.-S. (2014) Long-distance mechanism of neurotransmitter recycling mediated by glial network facilitates visual function in Drosophila. Proc. Natl. Acad. Sci. 111, 2812-2817.

(33) Borycz, J., Borycz, J. A., Edwards, T. N., Boulianne, G. L., and Meinertzhagen, I. A. (2012) The metabolism of histamine in the Drosophila optic lobe involves an ommatidial pathway: $\beta$-alanine recycles through the retina. J. Exp. Biol. 215, 1399-411.

(34) Stenesen, D., Moehlman, A. T., and Krämer, H. (2015) The carcinine transporter CarT is required in Drosophila photoreceptor neurons to sustain histamine recycling. Elife 4, e10972.

(35) Chaturvedi, R., Luan, Z., Guo, P., and Li, H.-S. (2016) Drosophila Vision Depends on Carcinine Uptake by an Organic Cation Transporter. Cell Rep. 14, 2076-83.

(36) Sitaraman, D., Zars, M., Laferriere, H., Chen, Y.-C., Sable-Smith, A., Kitamoto, T., Rottinghaus, G. E., and Zars, T. (2008) Serotonin is necessary for place memory in Drosophila. Proc. Natl. Acad. Sci. U. S. A. 105, 5579-84.

(37) Wagner, G. P. (2007) The developmental genetics of homology. Nat. Rev. Genet. 8, 473-9. 
Chapter 5. Conclusions and Future Directions 


\subsection{Conclusions}

In this work, I have developed and demonstrated CE-FSCV as a powerful technique for the analysis of neurotransmitters in extremely small tissue samples. The combination of capillary electrophoresis with fast scan cyclic voltammetry allows for quantification of neurotransmitter tissue content in single brains and central nervous systems of Drosophila. Single brain samples enable accurate understanding of the individual variance of neurotransmitter tissue content within strains and between strains of Drosophila. Samples as small as $0.016 \mathrm{mg}(16 \mu \mathrm{g})$ of tissue have been analyzed with no difficulty, and sample volumes used for analysis can be smaller than $2 \mu \mathrm{L}$ due to the use of electrokinetic injection.

In addition to the ability of CE-FSCV to handle small samples, CE-FSCV can be used for the sensitive detection of a wide range of electroactive neurotransmitters, ranging from catecholamines to histamine, without the need for sample pretreatment in the form of derivatization. Limits of detection as low as $4 \pm 1 \mathrm{pg}$ for histamine, $10 \pm 4 \mathrm{pg}$ for carcinine, $2.8 \pm 0.3 \mathrm{pg}$ for dopamine, and $9 \pm 3 \mathrm{pg}$ for $\mathrm{N}$ - $\beta$-alanyl-dopamine are possible through simple sample preparation. In the case of histamine and carcinine, this is particularly important as direct detection of carcinine had not been previously reported. This versatility allows for the simultaneous detection and quantification of a wide variety of neurotransmitters in a single tissue sample. In contrast to competing CE-EC methods, CE-FSCV provides an added layer of confidence to the identification of neurotransmitters through characteristic cyclic voltammograms. Compared to popular HPLC methods, CE-FSCV has a faster work flow due to the smaller sample sizes and volumes required for analysis. 
Overall, CE-FSCV is a powerful and versatile platform for the analysis of neurotransmitters in small samples. It is well suited to the small size of the Drosophila brain, but can easily be used for the analysis of a wide variety of tissues and sample types. In conjunction with the homebuilt nature of the CE-FSCV set up, this provides a platform for neurotransmitter analysis that is attractive to both analytical chemists and biologists interested in neurotransmitter analysis.

\subsection{Future Directions}

\subsubsection{Tissue Content Monitoring in Aging Larvae and Adults}

In Chapter 2, I determined the tissue content of tyramine, serotonin, octopamine, and dopamine in the larval CNS, the pupal brain, and the adult brain and compared them. Through this analysis and comparison of life stages, I found that in the pupal brain octopamine tissue content was below our limit of detection and tyramine was significantly higher than in either the larval or the adult brain. I could further use this method to monitor neurotransmitter tissue content in aging adult flies and in aging $3^{\text {rd }}$ instar larvae fed an ecdysone free diet (which prevents the larvae from pupation). Both the adult and ecdysone free larva may be useful for studies of aging and the impact of disease models or mutations on the aging process. ${ }^{1}$ There are a wide variety of Drosophila models of human aging related diseases, such as Parkinson's disease. Having a method to measure and compare neurotransmitter tissue content quickly in single flies is valuable, as it would assist in model validation studies.

In the case of Parkinson's disease, a variety of different models are available, targeting different genes and "kinds" of Parkinson's disease. ${ }^{2}$ These models have different phenotypes, and phenotype alone is insufficient for characterizing these flies. Measuring the single brain tissue content of dopamine in adults taken at different time periods post-eclosion from a colony of a given model (Parkin, for example) along with 
information on behavior of the flies and their mortality would demonstrate the specific effect of that model on those parameters over the life span of the adult fly. These values could then easily be compared to similar data obtained from colonies of different model flies. The study of tissue content in addition to strain mortality and life span information could prove to be valuable companions to imaging of DA neurons. Imagining of DA neurons, particularly important in Parkinson models, provides information on the number and presence of neurons. This is particularly important as Parkinson's models are expected to cause cell death of dopamine containing neurons, but tissue content studies could reveal if there is a compensatory increase in dopamine synthesis in remaining neurons that may not be obvious from imaging.

\subsubsection{Analysis of $\beta$-alanine in Drosophila and Development of a CE-FSCV Method for its Detection \\ In Chapter 3, I discussed monitoring the metabolism of histamine and carcinine}

in wild type flies and in metabolic mutants, ebony and tan. In these metabolic mutants, we found that the tissue content of the metabolite, carcinine, and not histamine, was most dramatically altered in tan mutants. [Another aspect of the histamine and carcinine metabolic cycle is $\beta$-alanine, which is required for the synthesis of carcinine from histamine and $\mathrm{N}$ - $\beta$-alanyl dopamine from dopamine. $\beta$-alanine is produced through two pathways in Drosophila: either from aspartate (black), which is the primary pathway or from uracil (pyd1, pyd2, and pyd3). ${ }^{3}$ There is also evidence of dietary availability of $\beta$ alanine, as yeast synthesize $\beta$-alanine. Analysis of $\beta$-alanine deficient mutants on a restricted diet to determine the tissue content of histamine and carcinine would allow us to further map the metabolic cycle of histamine and carcinine.

Development of a CE-FSCV method to detect $\beta$-alanine would allow us to track the metabolic cycle with greater fidelity and directly measure the impact of these mutations on the tissue content and availability of $\beta$-alanine in the tissue. Previous work 
from Borycz and coworkers indicates that $\beta$-alanine is detectable using electrochemical methods, and as such, no derivatization or other sample pretreatments to detect it would be required. ${ }^{3}$ Including the separation and detection of $\beta$-alanine would also allow us to monitor or detect tissue specific alterations in metabolite profiles or tissue storage. Ideally, separation and detection of $\beta$-alanine could be done using the same conditions for histamine, carcinine, dopamine, and N- $\beta$-alanyl dopamine. Simultaneous detection of all of these components would allow for analysis of all components of the metabolic pathway simultaneous and in the same individual sample.

\subsubsection{Histamine and Carcinine Content in Other Visual Mutants}

In Chapter 4, I analyzed the tissue content of histamine and carcinine in a wide variety of Drosophila melanogaster who lacked eyes (eye $e^{2}$ and eya $\left.{ }^{2}\right)$, lacked parts of the visual system $\left(s o^{1}\right)$, or had reduced visual functionality $\left(w^{1118}\right.$ and $\left.g \beta^{\beta}\right)$. In these visual mutants, we found that histamine tissue content, and not carcinine content, was lowered in visual mutants relative to Oregon $\mathrm{R}$ flies. These are primarily developmental mutants, where imaginal discs fail to mature into the correct adult structure during pupation. ${ }^{4}$ Other visual mutants exist, for example hdc (histidine decarboxylase deficient flies, who cannot synthesize histamine), or ninaB (flies who cannot produce the pigment necessary for visual transduction) ${ }^{5,6}$ These flies are not developmental mutants, and partial rescue of the blind phenotype is possible. Studying tissue content in these mutants and their tissue content in response to phenotype rescue (by feeding of histamine and all-trans retinal respectively) could provide further insight into the relationship between brain tissue content of histamine and how it is impacted by loss of visual stimulus.

\subsubsection{Development of a CE-FSCV Method for Quantification of Retinal, Retinoic Acid, and Retinoids}

In the case of ninaB flies or in flies with loss of retinol dehydrogenase it may be possible to monitor by CE-FSCV the tissue content of retinals and all-trans-retinal in eye 
tissue. ${ }^{6,7}$ All-trans-retinal detection by cyclic voltammetry has been previously documented, and there are a variety of HPLC methods developed for analysis of alltrans-retinal. ${ }^{8,9}$ Capillary electrophoresis methods for the separation of these compounds already exist; however, CE-EC methods have not been used in flies. ${ }^{10}$ In addition to its application to determining the presence of all-trans-retinal and related compounds in Drosophila, there is considerable interest in retinoids and retinoic acid in mammals. A CE-FSCV method for retinoid, retinal, and retinoic acid could be easily expanded to the analysis of blood or eye samples obtained from rats or mice.

\subsubsection{Histamine and Carcinine Content in RNAi Suppressed Drosophila}

In order to further develop the analysis of visual mutants I discussed in Chapter 4, development of more targeted and selective silencing or knock-out of the genes I previously worked on is necessary. Tissue specific knockout or knockdown would greatly enhance our ability to determine the effect of disrupted vision on the brain tissue content of histamine and carcinine. Developmental mutants often have a wide variety of knockon effects due to the important roles that the targeted genes play in transcription and other regulatory functions. In the case of the flies I analyzed in Chapter 4, sine oculis and glass are notable examples. They both encode transcription factors that play a large

role in the normal functioning and the development of the adult brain during pupation. ${ }^{11,12}$ As such, in hypomorph or null flies for these two genes in particular, there is a strong possibility that some of the effects I observed in tissue content of histamine and carcinine may be attributable to the effect of these transcription factors on the development of the brain rather than visual deficits alone. By the use of RNAi (RNA interference) to suppress the expression of these genes in specific targeted tissues such as the eye, eyelet, or ocelli of the adult it may be possible to better track and ascertain the specific effects of visual disruption on the brain tissue content of histamine and carcinine. ${ }^{13}$ This should also be done in eyeless and eyes absent flies to determine their 
contribution to normal brain development as well. Overall, RNAi would enable a more sophisticated and nuanced understanding of the link between brain histamine and visual function, without concerns over the effect of abnormal transcription factor function or abnormal interaction between these gene products on normal brain development.

\subsection{Summary}

My work with CE-FSCV has enabled exploration of new aspects of Drosophila development, the visual system in Drosophila, and the interaction between the visual system and the brain. Further development of CE-FSCV to further explore aging, neurotransmitter metabolism, and to expand to new classes of molecules present in biological systems would further increase the significance and accessibility of CE-FSCV. The expansion of CE-FSCV is possible due to the wide variety of FSCV detectable compounds, the unique identifying information available from cyclic voltammograms, and the low limits of detection provided by the unique combination of capillary electrophoresis and fast scan cyclic voltammetry. These factors are crucial for better understanding of systems as complex as Drosophila.

\subsection{References}

(1) Parkin, C. A., and Burnet, B. (1986) Growth arrest of Drosophila melanogaster on erg-2 and erg-6 sterol mutant strains of Saccharomyces cerevisiae. J. Insect Physiol. 32, 463-471.

(2) Whitworth, A. J. (2011) 1 - Drosophila Models of Parkinson's Disease (Theodore Friedmann, J. C. D., Ed.). Academic Press.

(3) Borycz, J., Borycz, J. A., Edwards, T. N., Boulianne, G. L., and Meinertzhagen, I. A. (2012) The metabolism of histamine in the Drosophila optic lobe involves an ommatidial pathway: $\beta$-alanine recycles through the retina. J. Exp. Biol. 215, 1399-411. 
(4) Wawersik, S. (2000) Vertebrate eye development as modeled in Drosophila. Hum. Mol. Genet. 9, 917-925.

(5) Melzig, J., Burg, M., Gruhn, M., Pak, W. L., and Buchner, E. (1998) Selective Histamine Uptake Rescues Photo- and Mechanoreceptor Function of Histidine Decarboxylase-Deficient Drosophila Mutant. J. Neurosci. 18, 7160-7166.

(6) Voolstra, O., Oberhauser, V., Sumser, E., Meyer, N. E., Maguire, M. E., Huber, A., and von Lintig, J. (2010) NinaB is essential for Drosophila vision but induces retinal degeneration in opsin-deficient photoreceptors. J. Biol. Chem. 285, 2130-9.

(7) Wang, X., Wang, T., Jiao, Y., von Lintig, J., and Montell, C. (2010) Requirement for an enzymatic visual cycle in Drosophila. Curr. Biol. 20, 93-102.

(8) Wring, S. A., Hart, J. P., and Knight, D. W. (1988) Voltammetric behaviour of alltrans-retinol (vitamin A1) at a glassy carbon electrode and its determination in human serum using high-performance liquid chromatography with electrochemical detection. Analyst 113, 1785.

(9) Kane, M. A., Folias, A. E., and Napoli, J. L. (2008) HPLC/UV quantitation of retinal, retinol, and retinyl esters in serum and tissues. Anal. Biochem. 378, 71-9.

(10) Bempong, D. K., Honigberg, I. L., and Meltzer, N. M. (1993) Separation of 13-cis and all-trans retinoic acid and their photodegradation products using capillary zone electrophoresis and micellar electrokinetic chromatography (MEC). J. Pharm. Biomed. Anal. 11, 829-833.

(11) Helfrich-Förster, C., Edwards, T., Yasuyama, K., Wisotzki, B., Schneuwly, S., Stanewsky, R., Meinertzhagen, I. A., and Hofbauer, A. (2002) The extraretinal eyelet of Drosophila: development, ultrastructure, and putative circadian function. J. Neurosci. 22, 
9255-66.

(12) Rieger, D., Stanewsky, R., and Helfrich-Förster, C. (2003) Cryptochrome, Compound Eyes, Hofbauer-Buchner Eyelets, and Ocelli Play Different Roles in the Entrainment and Masking Pathway of the Locomotor Activity Rhythm in the Fruit Fly Drosophila Melanogaster. J. Biol. Rhythms 18, 377-391.

(13) Martin, C. A., and Krantz, D. E. (2014) Drosophila melanogaster as a genetic model system to study neurotransmitter transporters. Neurochem. Int. 73, 71-88. 